\title{
1. GRAIN-SIZE, MORPHOLOGICAL, AND COMPOSITIONAL VARIATIONS IN IGNEOUS SILICATES IN MEDIUM-GRAINED DIABASE FROM HOLE 504B ${ }^{1}$
}

\author{
H.R. Naslund ${ }^{2}$
}

\begin{abstract}
Samples recovered from Hole 504B during Leg 140 include a number of medium-grained, holocrystalline diabases that appear to represent the cores of thick dikes. The plagioclase and pyroxene in these samples occur in a variety of crystal morphologies. Plagioclase occurs as phenocrysts, microphenocrysts, elongate crystals, skeletal crystals, and branching radial clusters. Pyroxene occurs as phenocrysts, microphenocrysts, ophitic crystals, and poikilitic crystals. Plagioclase compositions became progressively poorer in anorthite and $\mathrm{MgO}$ and progressively richer in $\mathrm{FeO}$ as crystallization proceeded, while the average grain volume decreased and the aspect ratio of individual grains increased. Pyroxene compositions are largely independent of crystal morphology.

The diabase dikes recovered from Hole 504B during Leg 140 appear to have crystallized in situ. Crystal compositions and morphologies are consistent with a rapid cooling rate and solidification times for individual dikes on the order of hours or days. The crystallization rate and nucleation rate of plagioclase lagged behind the cooling rate so that the degree of undercooling progressively increased as crystallization proceeded. Plagioclase crystal morphologies indicate much greater degrees of supersaturation than do pyroxene or olivine crystal morphologies. The 504B diabase magmas appear to have been emplaced with abundant preexisting pyroxene and olivine nuclei, but with few preexisting plagioclase nuclei. The suppression of plagioclase nucleation and crystallization relative to that of pyroxene and olivine could provide a mechanism by which the actual fractionation assemblage is more pyroxene-rich and plagioclase-poor than that predicted from thermodynamic models, or that observed in isothermal crystallization experiments.
\end{abstract}

\section{INTRODUCTION}

Samples recovered from Hole 504B during Legs 137 and 140 range from aphanitic dike margins to medium-grained diabase presumably from the centers of dikes. Within these samples, plagioclase and pyroxene are the dominant minerals, with lesser amounts of olivine, oxides, quartz, apatite, and alteration minerals. Plagioclase and pyroxene in the coarsest diabase samples have a variety of crystal morphologies and textures. Plagioclase occurs as: phenocrysts; microphenocrysts; elongate laths; skeletal crystals; and branching radial clusters of crystals. Pyroxene occurs as: phenocrysts; microphenocrysts; ophitic crystals; and large poikilitic crystals. Olivine in these samples occurs primarily as altered phenocrysts, but in some samples from the lower part of the section, unaltered olivine cores are preserved. This report will examine the relationship among grain size, crystal morphology, and composition of plagioclase, pyroxene, and olivine in the medium-grained diabase samples recovered from Hole 504B during Legs 137 and 140.

\section{TEXTURAL RELATIONSHIPS}

Plagioclase makes up $55 \%$ to $60 \%$ of the medium-grained diabase samples examined in this study. Plagioclase phenocrysts are the largest, but least abundant, crystal type. They make up 5\% to $10 \%$ of the total plagioclase. Phenocrysts are commonly complexly zoned with abundant crystal and melt inclusions. They range from 1 to $5 \mathrm{~mm}$ in length and are typically lath-shaped with aspect ratios between 1 and 3 (Table 1). In contrast, microphenocrysts, which make up $20 \%$ to $25 \%$ of the total plagioclase, range from 0.5 to $1 \mathrm{~mm}$ in length with aspect ratios between 2 and 4 . Plagioclase microphenocrysts are typically subhedral to euhedral with simple zoning patterns. Acicular crystals 0.8 to $1.5 \mathrm{~mm}$ in length with aspect ratios between 5 and 20 make up $50 \%$ to $60 \%$ of the total plagioclase. They range in morphol-

\footnotetext{
'Erzinger, J., Becker, K., Dick, H.J.B., and Stokking, L.B. (Eds.), 1995. Proc. ODP, Sci. Results, 137/140: College Station, TX (Ocean Drilling Program).

Department of Geological Sciences, State University of New York, Binghamton, NY 13902-6000, U.S.A.
}

ogy from euhedral elongate crystals to curved and skeletal crystals. They are listed separately in the tables as either elongate crystals, those that tend toward euhedral outlines, or skeletal crystals, those that tend toward curved, incipient-branching, or cellular outlines. An extreme example of branching crystals occurs as large radial clusters of plagioclase laths that radiate in bundles from a central point as fan-shaped or, in some cases, bow tie-shaped splays (Fig. 1). These plagioclase radial clusters typically make up $10 \%$ to $15 \%$ of the total plagioclase but were not observed in all of the medium-grained diabase samples examined. Individual laths within these radial clusters vary from euhedral to skeletal in shape, and are 1 to $2 \mathrm{~mm}$ in length with aspect ratios between 15 and 30 . Most crystals within radial clusters are narrow at the base of the cluster and become progressively wider toward their tips. Many radial clusters are enclosed within a single poikilitic pyroxene that appears to have precipitated simultaneously with the plagioclase radial cluster.

Augite makes up $35 \%$ to $40 \%$ of the medium-grained diabase samples examined in this study, but in many samples it has been partially altered, so its original modal abundance is hard to determine. Subhedral phenocrysts 1 to $3 \mathrm{~mm}$ across make up $2 \%$ to $3 \%$ of the total pyroxene. They are typically equidimensional with aspect ratios between 1 and 1.5 in most sections. A few crystals, however, are elongate with aspect ratios up to 6 . Some phenocrysts have abundant inclusions, but most have relatively inclusion-free cores with ophitic rims. Some of the pyroxene phenocrysts have sector-zoned and oscillatory-zoned cores (Fig. 2). The zoning is most pronounced in terms of the distribution of $\mathrm{Al}_{2} \mathrm{O}_{3}$ and $\mathrm{Cr}_{2} \mathrm{O}_{3}$. Small variations in $\mathrm{CaO}$ and $\mathrm{TiO}_{2}$ follow those of $\mathrm{Al}_{2} \mathrm{O}_{3}$, whereas minor variations in $\mathrm{MgO}$ are antithetic to those of $\mathrm{Al}_{2} \mathrm{O}_{3}$. Subhedral microphenocryst laths 0.5 to $1.0 \mathrm{~mm}$ in length with aspect ratios between 1 and 2 make up $5 \%$ to $10 \%$ of the total pyroxene. In most samples, $90 \%$ to $95 \%$ of the pyroxene occurs as ophitic to poikilitic crystals in the groundmass. Individual oikocrysts may be up to $4 \mathrm{~mm}$ across. Although these samples are distinctly porphyritic, there is a continuous variation in grain size between pyroxene phenocrysts on one extreme and groundmass poikilitic pyroxene on the other extreme (i.e., they are seriate porphyritic). The outer margins of pyroxene phenocrysts and microphenocrysts are typically ophitic or poikilitic with respect to the surrounding groundmass. Some of the 
Table 1. Average grain sizes.

\begin{tabular}{|c|c|c|c|}
\hline & $\begin{array}{l}\text { Length } \\
(\mathrm{mm})\end{array}$ & $\begin{array}{l}\text { Width } \\
(\mathrm{mm})\end{array}$ & $\begin{array}{c}\text { Aspect } \\
\text { ratio }\end{array}$ \\
\hline \multicolumn{4}{|l|}{ Plagioclase } \\
\hline Phenocryst & 1.84 & 0.87 & 2.29 \\
\hline Microphenocryst & 0.68 & 0.28 & 2.49 \\
\hline Elongate & 1.09 & 0.10 & 12.27 \\
\hline Skeletal & 1.16 & 0.13 & 11.17 \\
\hline Radial & 1.06 & 0.07 & 21.11 \\
\hline \multicolumn{4}{|l|}{ Pyroxene } \\
\hline Phenocryst & 1.94 & 1.57 & 1.27 \\
\hline Microphenocryst & 0.82 & 0.52 & 1.67 \\
\hline Ophitic & 1.06 & 0.84 & 1.29 \\
\hline Poikilitic & 2.59 & 1.52 & 1.95 \\
\hline \multicolumn{4}{|l|}{ Olivine } \\
\hline Phenocryst & 1.32 & 1.12 & 1.20 \\
\hline
\end{tabular}

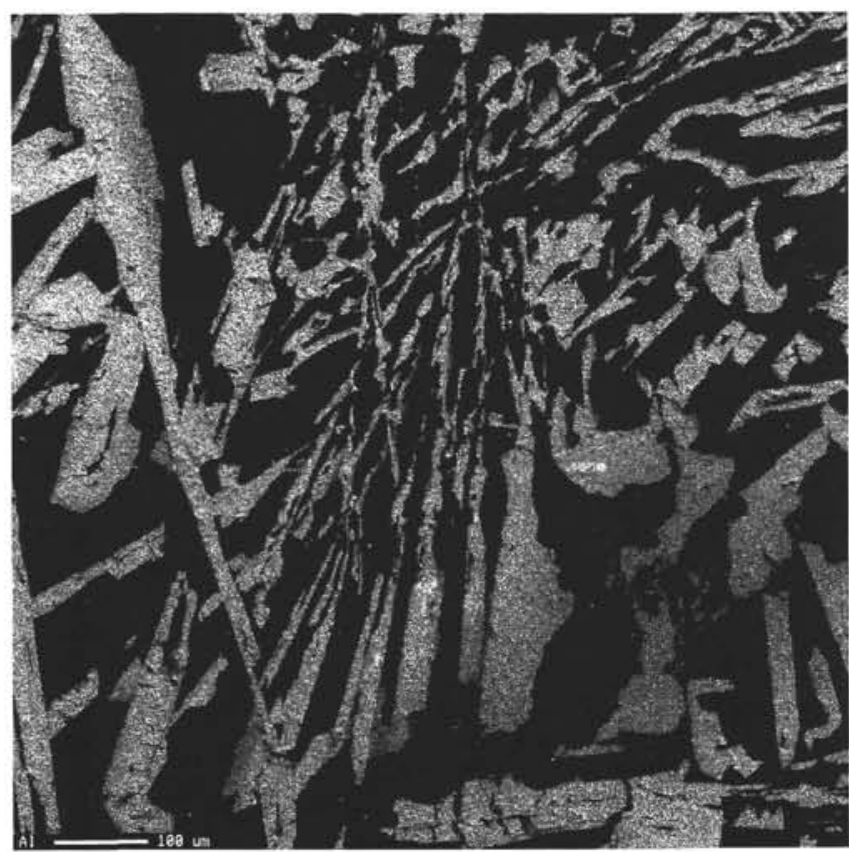

Figure 1. Distribution of $\mathrm{Al}_{2} \mathrm{O}_{3}$ in a branching radial cluster of plagioclase crystals surrounded by poikilitic pyroxene from Sample 140-504B-200R-1, $18-24 \mathrm{~cm}$. Image is an X-ray map generated using a JEOL 8900 electron microprobe in scan mode with 300,000 pixels and a dwell time of $50 \mathrm{~ms}$ per pixel. Black = low $\mathrm{Al}$ (surrounding pyroxene); gray = intermediate $\mathrm{Al}$; white $=$ highest $\mathrm{Al}$. Field of view is $0.89 \mathrm{~mm}$ wide.

more inclusion-rich phenocrysts are texturally similar to the larger poikilitic groundmass pyroxenes. In most of the samples examined, olivine occurs only as pseudomorphs replaced by alteration minerals. In a few samples, unaltered olivine cores are preserved. Olivine crystals range from 0.5 to $1.5 \mathrm{~mm}$ in diameter with low aspect ratios. Owing to the alteration present in the groundmass of almost all samples, the former existence of groundmass olivine would be hard to determine.

\section{COMPOSITIONAL VARIATIONS}

Plagioclase crystals in three samples (137-504B-181M-1, 115-124 $\mathrm{cm}$; 140-504B-200R-1, 18-24 cm; and 140-504B-226R-1, 8-12 cm) were analyzed using electron microprobe techniques (Tables 2-6). (Analyses were done on the JEOL 8900 at the Department of Geological Sciences, State University of New York at Binghamton, using

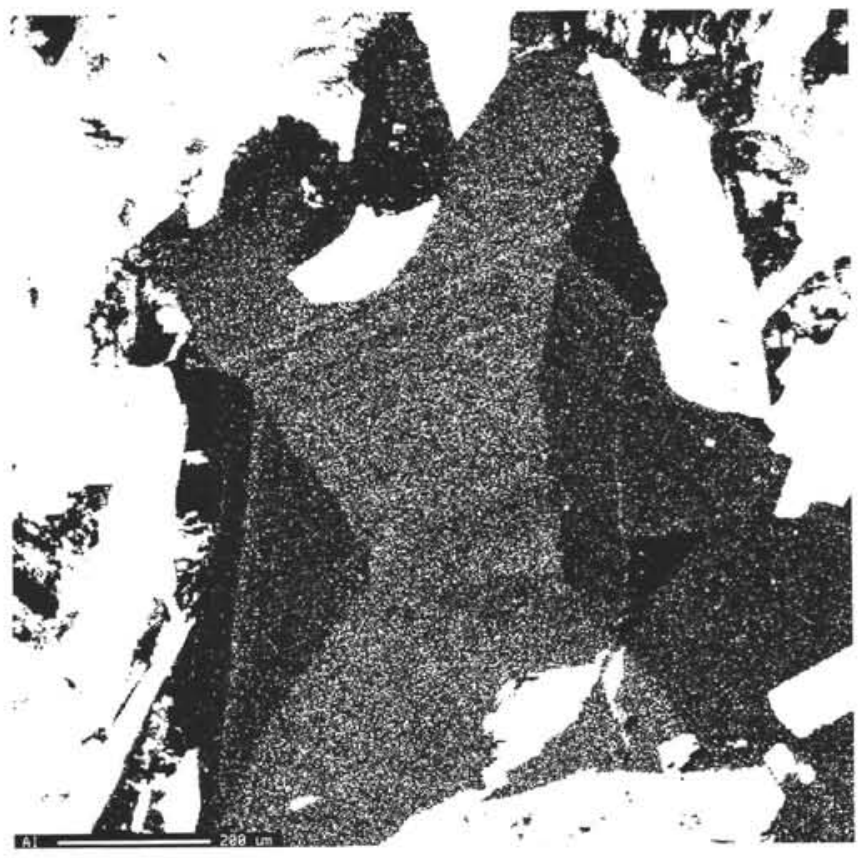

Figure 2. Distribution of $\mathrm{Al}_{2} \mathrm{O}_{3}$ in a sector-zoned and oscillatory-zoned pyroxene phenocryst core from Sample 140-504B-193R-1, 28-31 cm. Image is an X-ray map generated using a JEOL 8900 electron microprobe in scan mode with 300,000 pixels and a dwell time of $50 \mathrm{~ms}$ per pixel. Black = low $\mathrm{Al}$; gray $=$ moderate $\mathrm{Al}$; white $=$ highest $\mathrm{Al}$ (surrounding plagioclase crystals). Field of view is $1.11 \mathrm{~mm}$ wide.

wavelength dispersive spectrometers. Counting times were $30 \mathrm{~s}$ on peaks and $10 \mathrm{~s}$ on backgrounds, with a spot size of $<1$ micron, an accelerating voltage of $15 \mathrm{kv}$, and a sample current of $10 \mathrm{na}$. A ZAF correction scheme was used with oxide standards.) Data are divided into phenocrysts, microphenocrysts, elongate crystals, skeletal crystals, and plagioclase radial clusters. For most of the analyzed grains, traverses were made from margin to margin, and the data are separated into cores and rims. In the case of the plagioclase crystals within radial clusters, which do not have typical rims, the analyses are separated into those from the main mass of the crystal and those from the tips of the crystals. Figure 3 shows typical zoning patterns for traverses across the width of phenocrysts (Fig. 3A), microphenocrysts (Fig. 3B), and elongate crystals (Fig. 3C), and along the length of individual crystals within plagioclase radial clusters (Fig. 3D). The bulk of most crystals is composed of a relatively anorthite-rich core with a thin rim (or in the case of radial clusters, a small tip) of more anorthite-poor plagioclase. All groups show a systematic increase in $\mathrm{Fe}_{2} \mathrm{O}_{3}$ and decrease in $\mathrm{MgO}$ with decreasing anorthite content as has been observed in plagioclases from Hole 504B basalts (Sato, 1989). All five of the textural types analyzed fall on the same An\% $(\mathrm{Ca} / \mathrm{Ca}+$ $\mathrm{Na}+\mathrm{K}])$ vs. $\mathrm{Mg} \#(\mathrm{Mg} /[\mathrm{Mg}+\mathrm{Fe}])$ curve (Fig. 4A). There is, however a progressive decrease in An\% and $\mathrm{Mg} \#$ with increasing aspect ratio. Although there are a few stragglers, the cores of phenocrysts are generally between 80 and $90 \mathrm{An \%}$, the cores of microphenocrysts are generally between 75 and $90 \mathrm{An} \%$, the cores of elongate and skeletal crystals are generally between 70 and $80 \mathrm{An} \%$, and the cores of crystals within radial clusters are generally between 65 and 75 An\% (Fig. 4B). The few core compositions plotted near $50 \mathrm{An} \%$ may be the result of analyses of points that appear to be cores in thin section, but may be rims in a three-dimensional view. As is typical of other analyzed oceanic plagioclase (Bollinger and Semet, 1980; Thompson and Humphris, 1980), the plagioclases tend to be deficient in $\mathrm{Al}_{2} \mathrm{O}_{3}$ relative to $\mathrm{CaO}$ such that the anorthite content calculated by $\mathrm{Ca} /(\mathrm{Ca}+\mathrm{Na}+\mathrm{K})$ is $5 \%$ to $10 \%$ higher than that calculated by $(\mathrm{Al}-1) /(\mathrm{Si}+\mathrm{Al}-3)$. 
Table 2. Plagioclase phenocrysts.

\begin{tabular}{|c|c|c|c|c|c|c|c|c|c|c|c|c|c|c|c|c|c|c|c|}
\hline \multirow[b]{2}{*}{ Sample } & \multirow[b]{2}{*}{ Grain } & \multicolumn{8}{|c|}{ Weight percent } & \multicolumn{10}{|c|}{ Cations per 8 oxygens } \\
\hline & & $\mathrm{SiO}_{2}$ & $\mathrm{Al}_{2} \mathrm{O}_{3}$ & $\mathrm{O}_{3}$ & $\mathrm{CaO}$ & $\mathrm{MgO}$ & $\mathrm{Na}_{2} \mathrm{O}$ & $\mathrm{K}_{2} \mathrm{O}$ & Total & $\mathrm{Si}$ & Al & $\mathrm{Fe}$ & $\mathrm{Ca}$ & $\mathrm{Mg}$ & $\mathrm{Na}$ & K & Total & An\% & $\mathrm{Mg \#}$ \\
\hline $\begin{array}{l}\text { Plagioclase phenocryst cor } \\
\text { 137-504B- }\end{array}$ & & & & & & & & & & & & & & & & & & & \\
\hline $181 \mathrm{M}-1,115-124 \mathrm{~cm}$ & PH-1 & 47.29 & 31.71 & 0.401 & 17.23 & 0.269 & 1.20 & 0.004 & 98.10 & 2.211 & 1.748 & 0.014 & 0.863 & 0.019 & 0.108 & 0.000 & 4.963 & 88.8 & 57.1 \\
\hline $181 \mathrm{M}-1,115-124 \mathrm{~cm}$ & $\mathrm{PH}-1$ & 48.16 & 32.20 & 0.440 & 17.26 & 0.280 & 1.31 & 0.007 & 99.65 & 2.215 & 1.746 & 0.015 & 0.851 & 0.019 & 0.117 & 0.000 & 4.963 & 87.9 & 55.8 \\
\hline $181 \mathrm{M}-1,115-124 \mathrm{~cm}$ & $\mathrm{PH}-1$ & 47.68 & 32.68 & 0.519 & 17.18 & 0.245 & 1.40 & 0.004 & 99.71 & 2.194 & 1.773 & 0.018 & 0.847 & 0.017 & 0.125 & 0.000 & 4.974 & 87.1 & 48.3 \\
\hline $181 \mathrm{M}-1,115-124 \mathrm{~cm}$ & $\mathrm{PH}-2$ & $\begin{array}{l}49.34 \\
49.00\end{array}$ & 29.77 & 0.519 & 15.71 & 0.312 & 2.21 & 0.000 & 97.86 & 2.304 & 1.639 & 0.018 & 0.786 & 0.022 & 0.200 & 0.000 & 4.968 & 79.7 & 54.3 \\
\hline $181 \mathrm{M}-1,115-124 \mathrm{~cm}$ & $\mathrm{PH}-2$ & 47.30 & 30.80 & 0.427 & 17.00 & 0.263 & 1.44 & 0.007 & 97.23 & 2.232 & 1.713 & 0.015 & 0.860 & 0.019 & 0.131 & 0.000 & 4.970 & 86.7 & .0 \\
\hline 18 & $\mathrm{PH}-2$ & 48.84 & 30.65 & 0.413 & 16.16 & 0.306 & 1.97 & 0.000 & 3 & 271 & 1.680 & 0.015 & 0.805 & 0.021 & 0.178 & & & & 59.5 \\
\hline $\begin{array}{l}1811 \\
181\end{array}$ & $\mathrm{PH}-3$ & $\begin{array}{l}48.64 \\
49.07\end{array}$ & 32.59 & 0.573 & $\begin{array}{l}16.10 \\
16.61\end{array}$ & 0.298 & 1.58 & 0.011 & 0. & 8 & 1.744 & .020 & 0.8 & 0.0 & 0.139 & 0.0 & & & 50.7 \\
\hline $4 \mathrm{~cm}$ & $\mathrm{PH}-3$ & 47.20 & 33.04 & 0.509 & 17.57 & 0.224 & 1.12 & 0.000 & 99.67 & 2.174 & 1.795 & 0.018 & 0.867 & 0.015 & 0.100 & 0.000 & 4.97 & & 46.6 \\
\hline $181 \mathrm{M}-1,11$ & $\mathrm{PH}-3$ & 47.40 & 33.54 & 0.545 & 17.59 & 0.204 & 1.07 & 0.014 & 100.36 & 2.168 & 1.808 & 0.019 & 0.862 & 0.014 & 0.095 & 0.001 & 4.967 & 0 & 42.6 \\
\hline $181 \mathrm{M}-1,11$ & $\mathrm{PH}-3$ & 47.75 & 33.07 & 0.456 & 17.51 & 0.215 & 1.10 & 0.000 & 100.10 & 2.187 & 1.786 & 0.016 & 0.859 & 0.015 & 0.098 & 0.000 & 4.961 & 89.8 & 48.3 \\
\hline & PH-4 & 47.95 & 32.69 & 0.548 & 17.31 & 0.252 & 1.20 & 0.011 & 99.96 & 2.199 & 1.767 & 0.019 & 0.851 & 0.017 & 0.107 & 0.001 & 4.961 & 88.8 & 47.7 \\
\hline $181 \mathrm{M}-1,115-124 \mathrm{~cm}$ & $\mathrm{PH}-4$ & 48.50 & 32.30 & 0.504 & 16.66 & 0.314 & 1.55 & 0.008 & 99.83 & 2.223 & 1.746 & 0.017 & 0.818 & 0.022 & 0.137 & 0.001 & 4.964 & 85.6 & 55.2 \\
\hline $181 \mathrm{M}-1,115-124 \mathrm{~cm}$ & PH-4 & 48.82 & 32.17 & 0.455 & 16.56 & 0.342 & 1.62 & 0.003 & 99.96 & 2.234 & 1.735 & 0.016 & 0.812 & 0.023 & 0.143 & 0.000 & 4.963 & 85.0 & 59.8 \\
\hline $181 \mathrm{M}-1,115-124 \mathrm{~cm}$ & $\mathrm{PH}-4$ & 48.69 & 32.18 & 0.467 & 16.70 & 0.294 & 1.60 & 0.003 & 99.93 & 2.230 & 1.737 & 0.016 & 0.819 & 0.020 & 0.142 & 0.000 & 4.965 & 85.2 & 55.5 \\
\hline $140-504 \mathrm{~B}-$ & & & & & & & & & & & & & & & & & & & \\
\hline $200 \mathrm{R}-1,18$ & PH-1 & 47.47 & 33.93 & 0.475 & 17.59 & 0.192 & 1.36 & 0.012 & 101.04 & 2.159 & 1.819 & 0.016 & 0.857 & 0.013 & 0.120 & 0.001 & 4.984 & 87.6 & 44.5 \\
\hline $200 \mathrm{R}-1,18$ & $\mathrm{PH}-1$ & 47.47 & 32.86 & 0.486 & 17.20 & 0.231 & 10 & 0.012 & & 2.139 & 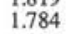 & 0.017 & 0.849 & 0.016 & 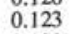 & 0.000 & & 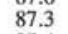 & 48.5 \\
\hline $200 \mathrm{R}-1,18-24 \mathrm{~cm}$ & $\mathrm{PH}-1$ & 47.68 & 32.38 & 0.487 & 17.26 & 0.197 & $\begin{array}{l}1.36 \\
1.36\end{array}$ & 0.007 & $\begin{array}{l}99.38 \\
99.02\end{array}$ & 2.201 & 1.762 & 0.017 & 0.854 & 0.014 & 0.122 & 0.000 & 4.970 & 87.4 & 44.5 \\
\hline $200 \mathrm{R}-1,18-24 \mathrm{~cm}$ & $\mathrm{PH}-2$ & $\begin{array}{l}48.64 \\
48.64\end{array}$ & 31.42 & $\begin{array}{l}0.461 \\
0.441\end{array}$ & 16.44 & 0.252 & $\begin{array}{l}1.30 \\
1.90\end{array}$ & 0.001 & 99.10 & 2.247 & 1.711 & 0.015 & 0.814 & 0.017 & 0.170 & 0.000 & 4.975 & 82.7 & 53.1 \\
\hline 2 & $\mathrm{PH}-2$ & $\begin{array}{l}8.04 \\
48.86\end{array}$ & $\begin{array}{l}3.42 \\
30.67\end{array}$ & 0.480 & $\begin{array}{l}10.44 \\
16.47\end{array}$ & $\begin{array}{l}0.252 \\
0.265\end{array}$ & & & & & 1.678 & 0.017 & & 0.018 & & & & 3.0 & \\
\hline 2 & $\mathrm{PH}-2$ & $\begin{array}{l}4.80 \\
49.87\end{array}$ & $\begin{array}{l}30.67 \\
31.69\end{array}$ & 0.491 & $\begin{array}{l}16.47 \\
16.33\end{array}$ & $\begin{array}{l}0.263 \\
0.301\end{array}$ & $\begin{array}{l}1.86 \\
2.17\end{array}$ & 0 & 10 & $\frac{8}{2}$ & $\begin{array}{l}1.678 \\
1.694\end{array}$ & 0.017 & $\begin{array}{l}0.819 \\
0.794\end{array}$ & 0.020 & 0.191 & 0.000 & $\begin{array}{l}4.968 \\
4.978\end{array}$ & .6 & 8 \\
\hline 200 & $\mathrm{PH}-2$ & 48.60 & 32.76 & 0.448 & 17.14 & 0. & 1.52 & 3 & & 2 & 1.757 & 0.015 & 0.836 & 0.015 & 0.134 & 0.0 & & 2 & 48.9 \\
\hline n & $\mathrm{PH}-2$ & 49.64 & 31.11 & 0.428 & 16.33 & 0.261 & 1.98 & 0.012 & & $=$ & 1.681 & 0.015 & 0.802 & 0.018 & 7 & 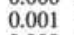 & & 2 & \\
\hline $\mathrm{m}$ & $\mathrm{PH}-2$ & 47.77 & 31.94 & 0.443 & 17.19 & 0.215 & 1.52 & 0.004 & 99.08 & 2.213 & 1.744 & 0.015 & 0.853 & 0.015 & & & & & \\
\hline $200 \mathrm{R}-1,18-24 \mathrm{~cm}$ & $\mathrm{PH}-2$ & 49.37 & 32.03 & 0.510 & 16.06 & 0.272 & 1.97 & 0.005 & 100.21 & 2.251 & 1.721 & 0.018 & 0.784 & 0.018 & 0.174 & 0.000 & 4.967 & 81.8 & 51.4 \\
\hline $200 \mathrm{R}-1,18-24 \mathrm{~cm}$ & $\mathrm{PH}-2$ & 49.46 & 31.19 & 0.512 & $\begin{array}{l}16.00 \\
16.13\end{array}$ & $\begin{array}{l}0.272 \\
0.306\end{array}$ & $\begin{array}{l}1.97 \\
1.97\end{array}$ & 0.000 & 99.57 & 2.270 & $\begin{array}{l}1.721 \\
1.688\end{array}$ & 0.018 & 0.793 & 0.021 & 0.175 & 0.000 & $\begin{array}{l}4.907 \\
4.965\end{array}$ & $\begin{array}{l}81.0 \\
81.9\end{array}$ & 54.2 \\
\hline $200 \mathrm{R}-1,18-24 \mathrm{~cm}$ & $\mathrm{PH}-2$ & 49.56 & 29.72 & 0.439 & 16.03 & 0.282 & 2.02 & 0.000 & 98.04 & 2.309 & 1.632 & 0.015 & 0.800 & 0.020 & 0.183 & 0.000 & 4.959 & 81.4 & 56.0 \\
\hline $200 \mathrm{R}-1,18-24 \mathrm{~cm}$ & $\mathrm{PH}-2$ & 49.81 & 30.94 & 0.451 & 16.38 & 0.301 & 2.02 & 0.012 & 99.90 & 2.280 & 1.669 & 0.016 & 0.803 & 0.021 & 0.179 & 0.001 & 4.968 & 81.7 & 9 \\
\hline $200 \mathrm{R}-1,18-24 \mathrm{~cm}$ & $\mathrm{PH}-2$ & 49.46 & 31.84 & 0.433 & 16.16 & 0.282 & 1.97 & 0.013 & 100. & 2.256 & 1.712 & 0.015 & 0.790 & 0.019 & 0.174 & 0.001 & 4.967 & 81.9 & 56.3 \\
\hline $1,18-24 \mathrm{~cm}$ & $\mathrm{PH}-2$ & 48.50 & 32.51 & 0.391 & 17.06 & 0.239 & 1.56 & & 10 & & 1.751 & 0.013 & 0.835 & 0.016 & 0.138 & 0.000 & 4.970 & 85.8 & 54.8 \\
\hline $18-24 \mathrm{~cm}$ & $\mathrm{PH}-2$ & 48.19 & 29.94 & 0.499 & 16.74 & 0.260 & 1.66 & 0.019 & 97 & 2. & 1.6 & 0.018 & 0.845 & 0.018 & 0.152 & 0.001 & & 84.7 & \\
\hline $18-24 \mathrm{~cm}$ & $\mathrm{PH}-2$ & 48.29 & 32.23 & 0.508 & 16.93 & $\begin{array}{l}0.269 \\
0.269\end{array}$ & 1.5 & & & 2. & 1.7 & 0.018 & & 0.018 & 0.141 & 0.001 & & 85.4 & 51.2 \\
\hline 2 & $\begin{array}{l}\mathrm{P} \\
\mathrm{P}\end{array}$ & 48.45 & 33.25 & 0.491 & & & & & & & & 0.0 & & & & & & 85.7 & \\
\hline 2 & $\begin{array}{l}P \\
P\end{array}$ & $\begin{array}{l}47.43 \\
47.98\end{array}$ & 32 & 0.8 & & & & & & & & 0.0 & & & & & & .5 & \\
\hline 200 & $\mathrm{PH}-2$ & $\begin{array}{l}4.98 \\
48.22\end{array}$ & $\begin{array}{l}3.2 .40 \\
31.59\end{array}$ & $\begin{array}{l}0.543 \\
0.478\end{array}$ & $\begin{array}{l}1.06 \\
16.83\end{array}$ & & $\begin{array}{l}1.7 \\
1 . \\
\text {. }\end{array}$ & & & 2. & 1.724 & 0.017 & 15 & & 0.147 & & & 5.0 & \\
\hline $\mathrm{cm}$ & $\mathrm{PH}-2$ & 49.73 & 29.64 & 0.553 & 15.00 & 0.271 & 2. & 0. & 97. & 2. & 1.6 & 0.019 & 0. & 9 & 0.222 & 0.000 & 4.964 & 7.1 & 49.2 \\
\hline & $\mathrm{PH}-3$ & 48.12 & 31.07 & 0.542 & 16.63 & 0.254 & 1.8 & 0.0 & 98 & 2. & 1.706 & 0.019 & 0.830 & 0.0 & 0.167 & & 4. & & \\
\hline $\mathrm{cm}$ & $\mathrm{PH}-3$ & 47.21 & 31.46 & 0.456 & 16.98 & 0.195 & 1.4 & 0.0 & 97. & 2.215 & 1.740 & 0.01 & 0.854 & 0. & 0. & & & & \\
\hline $4 \mathrm{~cm}$ & $\mathrm{PH}-3$ & 48.92 & 31.33 & 0.732 & 16.17 & 0.169 & 2. & 0.000 & 99. & 2.254 & 1.702 & 0.02 & 0.798 & 0.012 & 0.1 & & 4.9 & & \\
\hline $18-24 \mathrm{~cm}$ & $\mathrm{PH}$ & 49.03 & 31.74 & 0.598 & 16.32 & 0.229 & i. & 0.0 & 99. & 2. & 1.7 & 0.0 & 0.802 & 0.01 & 0.166 & 0.000 & 4.968 & 82.8 & 43.1 \\
\hline , $18-24 \mathrm{~cm}$ & $\mathrm{PH}$ & 48.07 & $\begin{array}{l}31.54 \\
31.53\end{array}$ & $\begin{array}{l}0.598 \\
0.456\end{array}$ & $\begin{array}{l}16.32 \\
16.70\end{array}$ & $\begin{array}{l}0.229 \\
0.210\end{array}$ & 1. & 0.0 & 98. & & 1.726 & 0.0 & $\begin{array}{l}0.802 \\
0.831\end{array}$ & 0.0 & $\begin{array}{l}0.100 \\
0.153\end{array}$ & 0.000 & $\begin{array}{l}4.908 \\
4.973\end{array}$ & $\begin{array}{l}84.4 \\
84.4\end{array}$ & $\begin{array}{l}43.1 \\
47.7\end{array}$ \\
\hline $1,18-24 \mathrm{~cm}$ & $\mathrm{PH}-3$ & 47.75 & 30.55 & 0.488 & 16.54 & 0.259 & 1.8 & 0 . & 97. & & 1.695 & 0.0 & 0.834 & 0. & 0.166 & 0.0 & 4.979 & 83.3 & 51.2 \\
\hline $1,18-24 \mathrm{~cm}$ & PH-4 & 46.89 & 32.31 & 0.493 & 16.34 & 0.223 & 2.03 & 0. & 98 & & 1.7 & 0.0 & 0.818 & 0.0 & 0.184 & 0.000 & 5.004 & 81.6 & 47.3 \\
\hline . & PH- & 46. & 32.18 & 0.556 & 16.54 & & & & & & & 0.0 & & & & & & & \\
\hline $18-24 c$ & $\mathrm{PH}$ & 46. & 32.42 & 0.6 & 16.32 & & & & & & 1.7 & 0.0 & & & & & & 82.3 & 41.2 \\
\hline $\mathrm{m}$ & $\mathrm{PH}-4$ & $\begin{array}{l}40.00 \\
46.17\end{array}$ & 32.59 & $\begin{array}{l}.049 \\
1.014\end{array}$ & 15.71 & & 1. & 0. & & & 1.796 & 0.0 & 0.787 & 0.0 & 0.165 & 0.6 & 5.0 & 2.6 & \\
\hline & $\mathrm{PH}-4$ & $\begin{array}{l}40.17 \\
46.96\end{array}$ & 31.75 & 0.578 & 16.62 & & & & & & 1.7 & 0.0 & 0.8 & & 0.157 & & & & \\
\hline 2 & $\mathrm{PH}-4$ & $\begin{array}{l}46.90 \\
47.39\end{array}$ & 31.61 & 0.4 & $\begin{array}{l}16.62 \\
16.23\end{array}$ & $\begin{array}{l}0.232 \\
0.222\end{array}$ & . 1. & 0. & & & i. & 0.0 & 0. & & 0.175 & & & 2 & \\
\hline 20 & $\mathrm{PH}-4$ & $\begin{array}{l}47.29 \\
47.29\end{array}$ & $\begin{array}{l}3.01 \\
33.37\end{array}$ & $\begin{array}{l}0.490 \\
0.592\end{array}$ & $\begin{array}{l}10 \\
16\end{array}$ & 0. & 1. & 0. & 99 & 2. & 1.8 & 0.8 & 0.8 & 0. & 0.161 & 0. & 4.5 & 83.4 & $\begin{array}{l}47.0 \\
43.9\end{array}$ \\
\hline & $\mathrm{PH}-4$ & 47.25 & 32.85 & 0.485 & 16. & 0.2 & 1. & 0. & & & & 0. & & & & & & & \\
\hline & & 46.02 & 33.02 & & 16 & & 1. & & & & & 0. & & & & & & & \\
\hline & & 45.97 & 32.88 & & 17 & & 1.5 & & & & & 0. & & & & & & & \\
\hline & & 46.12 & 32.42 & 0. & 17 & & 1. & & & & & & & & & & & & \\
\hline & & & & & & & & & & & & & & & & & & & \\
\hline & & & 32 & & 17 & & 1.3 & & & & & & & & & & & & \\
\hline 1 & & & 32 & 0. & 17 & & & & & & 1.8 & 0.0 & & & & & & & \\
\hline 4 & $\mathrm{PH}$ & 46. & 32.26 & & 16.76 & 0.287 & & 0. & & & & 0.0 & & 0.8 & & & & & \\
\hline 1824 & PH-5 & 46.53 & 33.11 & 0.4 & 16.83 & & & & & & & 0.0 & 0.8 & 0.0 & 0.143 & & 4.992 & 85.4 & \\
\hline $18-24 \mathrm{~cm}$ & PH-5 & 49.77 & 29.06 & 0.5 & 15.48 & 0.307 & & 0.0 & & 2 & 1.6 & 0.021 & 0.776 & 0.0 & 0.228 & 0.000 & 4.9 & 77.3 & \\
\hline $18-24 \mathrm{~cm}$ & $\mathrm{PH}-5$ & 46.92 & 32.11 & 0.459 & $\begin{array}{l}16.49 \\
16.49\end{array}$ & 0.254 & 1.70 & 0.0 & 9 & 2.197 & 1.773 & 0.016 & 0.827 & 0.0 & 0.154 & 0.001 & 4.9 & 84 & 52.3 \\
\hline & $\mathrm{PH}$ & $\begin{array}{l}40.92 \\
47.71\end{array}$ & 31.17 & $\begin{array}{l}0.455 \\
0.454\end{array}$ & $\begin{array}{l}15.48 \\
15.38\end{array}$ & $\begin{array}{l}0.254 \\
0.273\end{array}$ & & & & & 1.727 & 0.016 & 0.775 & 0.0 & 0.213 & 0.000 & 4.9 & $\begin{array}{l}78.4 \\
78.2\end{array}$ & \\
\hline $8-12 c$ & $\mathrm{PH}-1$ & 47.13 & 32.54 & $\begin{array}{l}0.454 \\
0.550\end{array}$ & $\begin{array}{l}16.18 \\
16.18\end{array}$ & 0.341 & 2. & 0.0 & 98 & 2.1 & 1.782 & 0.019 & 0.806 & 0.024 & 0.180 & 0.000 & $\begin{array}{l}4.9 \\
5.0\end{array}$ & 81.7 & \\
\hline $8-12 c$ & $\mathrm{PH}-2$ & $\begin{array}{l}4.13 \\
45.57\end{array}$ & 33.44 & $\begin{array}{l}0.350 \\
0.352\end{array}$ & $\begin{array}{l}17.18 \\
17.13\end{array}$ & $\begin{array}{l}0.341 \\
0.184\end{array}$ & 1.4. & 0.6 & & 2.1 .1 & $\begin{array}{l}1.782 \\
1.848\end{array}$ & 0.012 & $\begin{array}{l}0.800 \\
0.860\end{array}$ & 0.013 & 0.132 & 0.000 & 5.0 & $\begin{array}{l}81.1 \\
86.7\end{array}$ & $\begin{array}{l}53.1 \\
50.9\end{array}$ \\
\hline$-12 \mathrm{ct}$ & $\mathrm{PH}-2$ & 45.08 & 33.17 & 0.3 & 17.04 & & i. & & & & 1.8 & 0. & & & & & & 3 & \\
\hline & & 45.0 & & & & & & & & & & & & & & & & & \\
\hline & & & & & & & & & & & & & & & & & & & \\
\hline & & 74 & & & & 237 & 1.58 & & & 180 & & 14 & 0.841 & 016 & 142 & .000 & .988 & 85.5 & .3 \\
\hline
\end{tabular}

Plagioclase phenocryst rims 137-504B-

$\begin{array}{llllllllll}181 \mathrm{M}-1,115-124 \mathrm{~cm} & \text { PH-1r } & 52.89 & 28.70 & 0.958 & 13.20 & 0.179 & 3.81 & 0.003 & 99.74\end{array}$

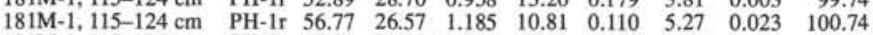

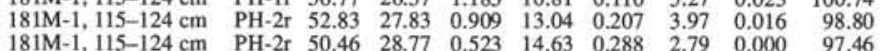

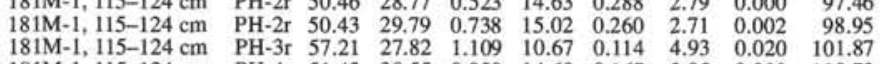



\section{0-504B-}

$200 \mathrm{R}-1,18-24 \mathrm{~cm}$ 200R-1, $18-24 \mathrm{~cm}$ $200 \mathrm{R}-1,18-24 \mathrm{~cm}$ $200 \mathrm{R}-1,18-24 \mathrm{~cm}$ $200 \mathrm{R}-1,18-24 \mathrm{~cm}$ 200R $1,18-24 \mathrm{~cm}$ $200 \mathrm{R}-1,18-24 \mathrm{~cm}$ $226 \mathrm{R}-1,8-12 \mathrm{~cm}$ 226R-1, 8-12 cm $226 \mathrm{R}-1,8-12 \mathrm{~cm}$ $226 \mathrm{R}-1,8-12 \mathrm{~cm}$
$226 \mathrm{R}-1,8-12 \mathrm{~cm}$

$\begin{array}{lllllllll}\text { PH-2r } & 53.41 & 28.25 & 0.926 & 13.04 & 0.188 & 3.90 & 0.002 & 99.71\end{array}$

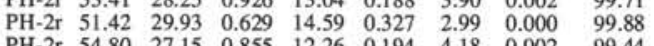
$\begin{array}{llllllllr}\text { PH-3r } & 54.92 & 27.84 & 1.301 & 12.08 & 0.171 & 4.38 & 0.009 & 100.70\end{array}$ $\begin{array}{llllllllr}\text { PH-3r } & 50.29 & 29.44 & 0.543 & 15.39 & 0.316 & 2.49 & 0.001 & 98.47\end{array}$ $\begin{array}{lllllllll}\text { PH-4r } & 52.93 & 27.41 & 1.228 & 11.44 & 0.188 & 4.50 & 0.006 & 97.71\end{array}$ $\begin{array}{llllllll}\text { PH-5r } & 48.67 & 30.02 & 0.849 & 14.75 & 0.147 & 2.78 & 0.009\end{array}$ $\begin{array}{llllllll}\text { PH-1r } & 52.92 & 27.80 & 0.882 & 11.99 & 0.151 & 4.38 & 0.027\end{array}$ $\begin{array}{llllllll}\text { PH-1r } & 49.86 & 29.31 & 0.749 & 14.12 & 0.247 & 3.26 & 0.015 \\ \text { PH-2r } & 55.36 & 26.95 & 1.107 & 11.12 & 0.126 & 5.12 & 0.030\end{array}$ $\begin{array}{llllllll}\text { PH-2r } & 54.82 & 27.37 & 1.120 & 11.52 & 0.128 & 4.91 & 0.006\end{array}$ $\begin{array}{cccccccc}\text { PH-3r } & 57.30 & 25.33 & 0.693 & 9.20 & 0.060 & 5.96 & 0.044 \\ \text { PH-3r } & 50.71 & 29.96 & 0.939 & 14.04 & 0.247 & 3.14 & 0.002\end{array}$ $\begin{array}{llllllllll}2211 & 1.748 & 0.014 & 0.863 & 0.019 & 0.108 & 0.000 & 4.963 & 88.8\end{array}$ 57.1 $87.1 \quad 48.3$

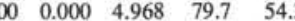
$\begin{array}{llllllllll}2.232 & 1.713 & 0.015 & 0.860 & 0.019 & 0.131 & 0.000 & 4.970 & 86.7 & 55.0\end{array}$ $\begin{array}{lllllllllll}2.271 & 1.680 & 0.015 & 0.805 & 0.021 & 0.178 & 0.000 & 4.970 & 81.9 & 59.5 \\ 2.228 & 1.744 & 0.020 & 0.808 & 0.020 & 0.139 & 0.001 & 4.960 & 85.2 & 50.7\end{array}$ $\begin{array}{lllllllllll}2.174 & 1.795 & 0.018 & 0.867 & 0.015 & 0.100 & 0.000 & 4.970 & 89.6 & 46.6\end{array}$ $\begin{array}{lllllllllll}2.168 & 1.808 & 0.019 & 0.862 & 0.014 & 0.095 & 0.001 & 4.967 & 90.0 & 42.6 \\ 2.187 & 1.786 & 0.016 & 0.859 & 0.015 & 0.098 & 0.000 & 4.961 & 89.8 & 48.3\end{array}$ $\begin{array}{llllllllll}2.199 & 1.767 & 0.019 & 0.851 & 0.017 & 0.107 & 0.001 & 4.961 & 88.8 & 47.7\end{array}$ $\begin{array}{llllllllll}2.234 & 1.735 & 0.016 & 0.812 & 0.023 & 0.143 & 0.000 & 4.963 & 85.0 & 59.8\end{array}$ $\begin{array}{llllllllll}2.159 & 1.819 & 0.016 & 0.857 & 0.013 & 0.120 & 0.001 & 4.984 & 87.6 & 44.5\end{array}$

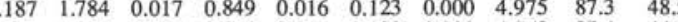
$\begin{array}{lllllllllll}2.201 & 1.762 & 0.017 & 0.854 & 0.014 & 0.122 & 0.000 & 4.970 & 87.4 & 44.5 \\ 2.247 & 1.711 & 0.015 & 0.814 & 0.017 & 0.170 & 0.000 & 4.975 & 82.7 & 53.1\end{array}$ $\begin{array}{lllllllllll}2.268 & 1.678 & 0.017 & 0.819 & 0.018 & 0.167 & 0.000 & 4.968 & 83.0 & 52.2\end{array}$ $\begin{array}{lllllllllll}2.262 & 1.694 & 0.017 & 0.794 & 0.020 & 0.191 & 0.000 & 4.978 & 80.6 & 54.8 \\ 2.212 & 1.757 & 0.015 & 0.836 & 0.015 & 0.134 & 0.000 & 4.969 & 86.2 & 48.9\end{array}$ $\begin{array}{lllllllllll}2.275 & 1.681 & 0.015 & 0.802 & 0.018 & 0.175 & 0.001 & 4.966 & 82.0 & 54.7\end{array}$ $\begin{array}{lllllllllll}2.213 & 1.744 & 0.015 & 0.853 & 0.015 & 0.136 & 0.000 & 4.976 & 86.2 & 49.0\end{array}$ $\begin{array}{llllllllll}2.270 & 1.688 & 0.018 & 0.793 & 0.021 & 0.175 & 0.000 & 4.965 & 81.9 & 54.2\end{array}$ $\begin{array}{llllllllll}2.309 & 1.632 & 0.015 & 0.800 & 0.020 & 0.183 & 0.000 & 4.959 & 81.4 & 56.0\end{array}$ $\begin{array}{lllllllllll}2.256 & 1.712 & 0.015 & 0.790 & 0.019 & 0.174 & 0.001 & 4.967 & 81.9 & 56.3\end{array}$ $\begin{array}{llllllllll}2.216 & 1.751 & 0.013 & 0.835 & 0.016 & 0.138 & 0.000 & 4.970 & 85.8 & 54.8\end{array}$ $\begin{array}{llllllllll}2.278 & 1.662 & 0.018 & 0.845 & 0.018 & 0.152 & 0.001 & 4.966 & 84.7 & 50.8\end{array}$ $\begin{array}{lllllllllll}2.198 & 1.778 & 0.017 & 0.828 & 0.013 & 0.138 & 0.000 & 4.973 & 85.7 & 44.4\end{array}$ $\begin{array}{lllllllllll}2.207 & 1.757 & 0.019 & 0.841 & 0.011 & 0.142 & 0.000 & 4.976 & 85.5 & 37.0 \\ 2.233 & 1.724 & 0.017 & 0.835 & 0.015 & 0.147 & 0.000 & 4.970 & 85.0 & 46.9\end{array}$ $\begin{array}{lllllllllll}2.322 & 1.631 & 0.019 & 0.751 & 0.019 & 0.222 & 0.000 & 4.964 & 77.1 & 49.2\end{array}$ $\begin{array}{lllllllllll}2.241 & 1.706 & 0.019 & 0.830 & 0.018 & 0.167 & 0.000 & 4.981 & 83.2 & 48 .\end{array}$ $\begin{array}{llllllllll}2.254 & 1.702 & 0.025 & 0.798 & 0.012 & 0.185 & 0.000 & 4.975 & 81.2 & 31.4\end{array}$ $\begin{array}{llllllllll}2.248 & 1.715 & 0.021 & 0.802 & 0.016 & 0.166 & 0.000 & 4.968 & 82.8 & 43.1\end{array}$ $\begin{array}{lllllllllll}2.248 & 1.695 & 0.017 & 0.834 & 0.018 & 0.166 & 0.001 & 4.979 & 83.3 & 51.2\end{array}$ $\begin{array}{llllllllll}2.190 & 1.779 & 0.017 & 0.818 & 0.016 & 0.184 & 0.000 & 5.004 & 81.6 & 47.3\end{array}$ $\begin{array}{llllllllll}2.191 & 1.773 & 0.020 & 0.828 & 0.017 & 0.168 & 0.000 & 4.997 & 83.1 & 46.7\end{array}$

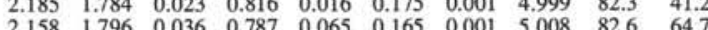
$\begin{array}{llllllllll}2.203 & 1.756 & 0.020 & 0.835 & 0.016 & 0.157 & 0.001 & 4.988 & 84.1 & 44.3\end{array}$ $\begin{array}{llllllllll}2.219 & 1.745 & 0.017 & 0.814 & 0.015 & 0.175 & 0.001 & 4.988 & 82.2 & 47.0 \\ 2.175 & 1.810 & 0.020 & 0.807 & 0.016 & 0.161 & 0.000 & 4.990 & 83.4 & 43.9\end{array}$ $\begin{array}{lllllllllll}2.190 & 1.795 & 0.017 & 0.807 & 0.016 & 0.156 & 0.000 & 4.982 & 83.8 & 47.9\end{array}$ $\begin{array}{llllllllll}2.155 & 1.823 & 0.016 & 0.851 & 0.013 & 0.134 & 0.001 & 4.993 & 86.3 & 4.9\end{array}$ $\begin{array}{llllllllll}2.165 & 1.794 & 0.019 & 0.859 & 0.017 & 0.147 & 0.000 & 5.001 & 85.4 & 48.1\end{array}$ $\begin{array}{llllllllll}2.145 & 1.823 & 0.020 & 0.870 & 0.014 & 0.126 & 0.000 & 4.997 & 87.3 & 40.9\end{array}$ $\begin{array}{lllllllllll}2.168 & 1.800 & 0.015 & 0.858 & 0.016 & 0.131 & 0.000 & 4.989 & 86.8 & 52.0\end{array}$ $\begin{array}{llllllllll}2.185 & 1.779 & 0.018 & 0.840 & 0.020 & 0.146 & 0.000 & 4.989 & 85.2 & 52.9\end{array}$ $\begin{array}{llllllllll}2.164 & 1.815 & 0.017 & 0.839 & 0.014 & 0.143 & 0.000 & 4.992 & 85.4 & 44.5\end{array}$

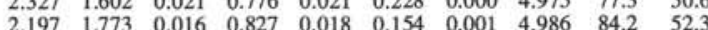
$\begin{array}{lllllllllll}2.242 & 1.727 & 0.016 & 0.775 & 0.019 & 0.213 & 0.000 & 4.993 & 78.4 & 54.4\end{array}$ $\begin{array}{llllllllll}2.189 & 1.782 & 0.019 & 0.806 & 0.024 & 0.180 & 0.000 & 5.000 & 81.7 & 55.1 \\ 2.135 & 1.848 & 0.012 & 0.860 & 0.013 & 0.132 & 0.000 & 5.001 & 86.7 & 50.9\end{array}$ $\begin{array}{lllllllllll}2.132 & 1.849 & 0.013 & 0.863 & 0.016 & 0.125 & 0.001 & 4.999 & 87.3 & 54.5\end{array}$ $\begin{array}{lllllllllll}2.195 & 1.773 & 0.015 & 0.841 & 0.018 & 0.138 & 0.000 & 4.980 & 85.9 & 55.0\end{array}$

$\begin{array}{llllllllll}2.408 & 1.540 & 0.033 & 0.644 & 0.012 & 0.336 & 0.000 & 4.974 & 65.7 & 27.0\end{array}$ $\begin{array}{llllllllll}2.541 & 1.402 & 0.040 & 0.518 & 0.007 & 0.458 & 0.001 & 4.968 & 53.0 & 15.5\end{array}$ $\begin{array}{llllllllll}2.428 & 1.508 & 0.031 & 0.642 & 0.014 & 0.354 & 0.001 & 4.979 & 64.4 & 31.1\end{array}$ $\begin{array}{lllllllllll}2.358 & 1.585 & 0.018 & 0.733 & 0.020 & .253 & 0.000 & 4.967 & 74.3 & 52.2\end{array}$ $\begin{array}{llllllllll}2.326 & 1.620 & 0.026 & 0.742 & 0.018 & 0.242 & 0.000 & 4.973 & 75.4 & 41.1 \\ 2.525 & 1.447 & 0.037 & 0.504 & 0.008 & 0.422 & 0.001 & 4.945 & 54.4 & 16.9\end{array}$ $\begin{array}{lllllllllll}2.327 & 1.630 & 0.032 & 0.713 & 0.011 & 0.258 & 0.000 & 4.971 & 73.4 & 26.3\end{array}$

$\begin{array}{lllllllllll}2.430 & 1.515 & 0.032 & 0.636 & 0.013 & 0.344 & 0.000 & 4.969 & 64.9 & 28.7\end{array}$ $\begin{array}{llllllllll}2.344 & 1.608 & 0.022 & 0.712 & 0.022 & 0.264 & 0.000 & 4.973 & 72.9 & 50.7\end{array}$ $\begin{array}{llllllllll}2.490 & 1.454 & 0.029 & 0.597 & 0.013 & 0.369 & 0.000 & 4.952 & 61.8 & 31.0\end{array}$ $\begin{array}{lllllllllll}2.468 & 1.475 & 0.044 & 0.582 & 0.011 & 0.382 & 0.001 & 4.963 & 60.3 & 20.7\end{array}$ $\begin{array}{lllllllllll}2.468 & 1.475 & 0.044 & 0.582 & 0.011 & 0.382 & 0.001 & 4.963 & 60.3 & 20.7 \\ 2.331 & 1.608 & 0.019 & 0.764 & 0.022 & 0.224 & 0.000 & 4.968 & 77.3 & 53.5\end{array}$ $\begin{array}{lllllllllll}2.331 & .608 & 0.019 & 0.764 & 0.022 & 0.224 & 0.000 & 4.968 & 77.3 & 53.5 \\ 2.453 & 1.498 & 0.043 & 0.568 & 0.013 & 0.404 & 0.000 & 4.979 & 58.4 & 23.3\end{array}$ $\begin{array}{llllllllll}2.453 & 1.498 & 0.043 & 0.568 & 0.013 & 0.404 & 0.000 & 4.979 & 58.4 & 23.3 \\ 2.289 & 1.664 & 0.030 & 0.743 & 0.010 & 0.253 & 0.001 & 4.991 & 74.5 & 25.5\end{array}$ $\begin{array}{llllllllll}2.289 & 1.664 & 0.030 & 0.743 & 0.010 & 0.253 & 0.001 & 4.991 & 74.5 & 25.5 \\ 2.442 & 1.513 & 0.031 & 0.593 & 0.010 & 0.392 & 0.002 & 4.983 & 60.1 & 25.3\end{array}$ $\begin{array}{llllllllll}2.442 & 1.513 & 0.031 & 0.593 & 0.010 & 0.392 & 0.002 & 4.983 & 60.1 & 25.3 \\ 2.332 & 1.616 & 0.026 & 0.707 & 0.017 & 0.295 & 0.001 & 4.995 & 70.5 & 39.5\end{array}$ $\begin{array}{llllllllll}2.332 & 1.616 & 0.026 & 0.707 & 0.017 & 0.295 & 0.001 & 4.995 & 70.5 & 39.5 \\ 2.506 & 1.438 & 0.038 & 0.540 & 0.008 & 0.449 & 0.002 & 4.981 & 54.5 & 18.4\end{array}$ $\begin{array}{llllllllll}2.506 & 1.438 & 0.038 & 0.540 & 0.008 & 0.449 & 0.002 & 4.981 & 54.5 & 18.4 \\ 2.483 & 1.462 & 0.038 & 0.559 & 0.009 & 0.431 & 0.000 & 4.983 & 56.4 & 18.4\end{array}$ $\begin{array}{lllllllllll}2.606 & 1.358 & 0.024 & 0.448 & 0.004 & 0.525 & 0.003 & 4.967 & 45.9 & 14.6\end{array}$ $\begin{array}{lllllllllll}2.333 & 1.625 & 0.033 & 0.692 & 0.017 & 0.280 & 0.000 & 4.979 & 71.2 & 34.3\end{array}$ 
Table 3. Plagioclase microphenocrysts.

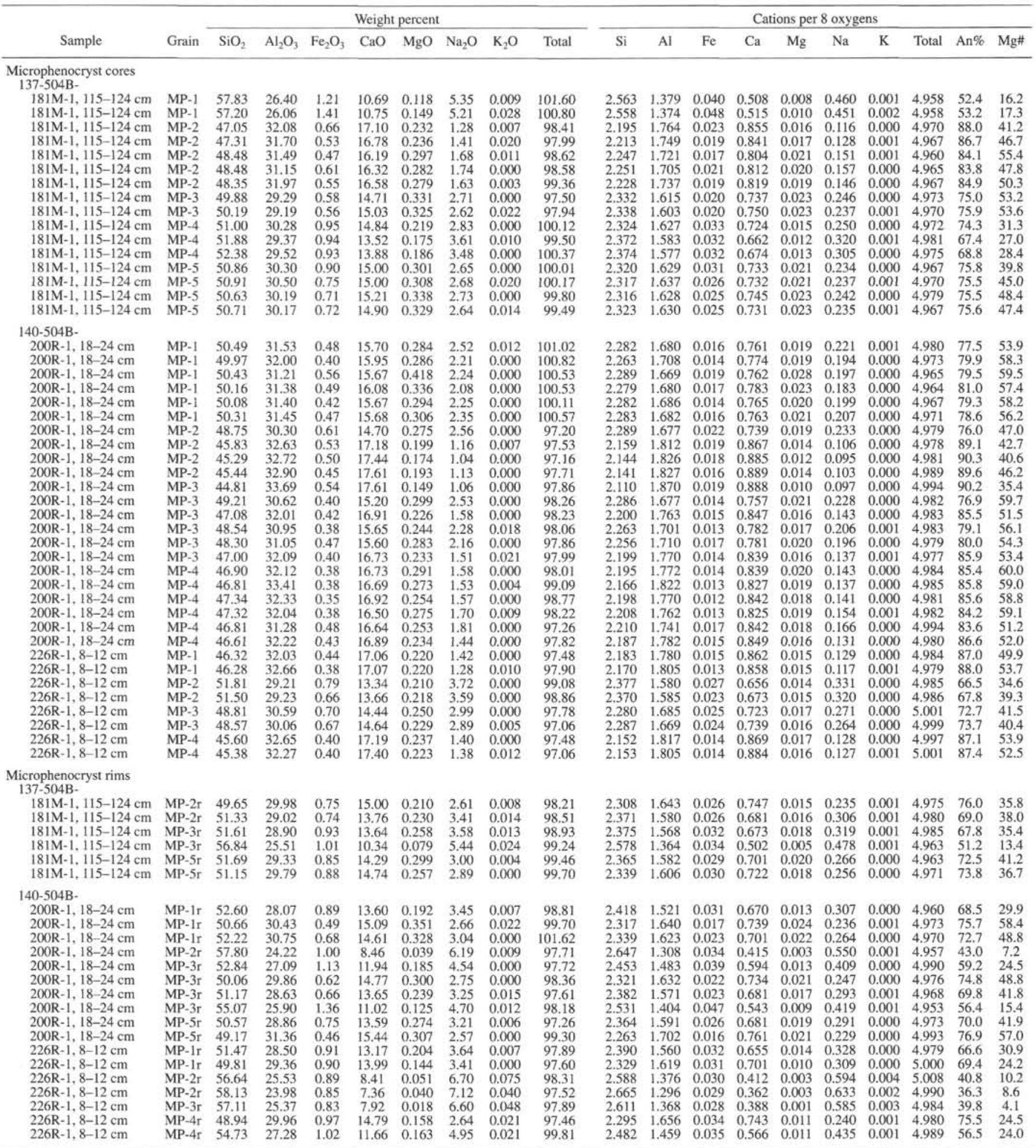

Pyroxene crystals in three samples were analyzed using electron microprobe techniques (Tables 7-10). Data are divided into phenocrysts, microphenocrysts, ophitic crystals, and poikilitic crystals. For most of the analyzed grains, traverses were made from margin to margin, with the data from visible rims indicated in the tables by an "r." The pyroxenes in the medium-grained diabase samples examined are strongly zoned; $\mathrm{Mg \# s} \mathrm{range} \mathrm{from} 20$ to 87 , with most of the core compositions in the range of 70 to 84 . These values are in agreement with the $\mathrm{Mg} \#$ s for augite observed during the experimental crystallization of abyssal tholeiites at low pressure (Walker et al., 1979; Grove and Bryan, 1983; Bloomer et al., 1989). All of the pyroxenes analyzed are low in $\mathrm{Na}_{2} \mathrm{O}$ and $\mathrm{Al}_{2} \mathrm{O}_{3}$, as is typical of oceanic and 
Table 4. Elongate plagioclase.

\begin{tabular}{|c|c|c|c|c|c|c|c|c|c|c|c|c|c|c|c|c|c|c|c|}
\hline \multirow[b]{2}{*}{ Sample } & \multirow[b]{2}{*}{ Grain } & \multicolumn{8}{|c|}{ Weight percent } & & & & & ons & 8 oxyge & & & & \\
\hline & & $\mathrm{SiO}_{2}$ & $\mathrm{Al}_{2} \mathrm{O}_{3}$ & $\mathrm{Fe}_{2} \mathrm{O}_{3}$ & $\mathrm{CaO}$ & $\operatorname{lgO}$ & $\mathrm{Na}_{2} \mathrm{O}$ & $\mathrm{K}_{2} \mathrm{O}$ & Total & $\mathrm{Si}$ & Al & $\mathrm{Fe}$ & $\mathrm{Ca}$ & $\mathrm{Mg}$ & $\mathrm{Na}$ & K & Total & An\% & $\mathrm{Mg} \#$ \\
\hline $\begin{array}{l}\text { Elongate plagioclase cores } \\
137-504 \mathrm{~B}-\end{array}$ & & & & & & & & & & & & & & & & & & & \\
\hline $181 \mathrm{M}-1,115-124 \mathrm{~cm}$ & EL-1 & 51.27 & 29.52 & 0.968 & 14.77 & 0.292 & 2.97 & 0.007 & 99.80 & 2.344 & 1.591 & 0.033 & 0.724 & 0.020 & 0.263 & 0.000 & 4.976 & 73.3 & 37.4 \\
\hline $181 \mathrm{M}-1,115-124 \mathrm{~cm}$ & EL-1 & 51.00 & 29.55 & 0.821 & 14.62 & 0.266 & 2.80 & 0.011 & 99.07 & 2.345 & 1.602 & 0.028 & 0.720 & 0.018 & 0.250 & 0.001 & 4.965 & 74.2 & 39.1 \\
\hline $181 \mathrm{M}-1,115-124 \mathrm{~cm}$ & EL-2 & 51.34 & 28.71 & 0.915 & 14.41 & 0.239 & 2.95 & 0.003 & 98.56 & 2.372 & 1.564 & 0.032 & 0.713 & 0.017 & 0.264 & 0.000 & 4.962 & 73.0 & 34.1 \\
\hline $181 \mathrm{M}-1,115-124 \mathrm{~cm}$ & EL-3 & 50.74 & 30.73 & 0.714 & 15.22 & 0.359 & 2.60 & 0.000 & 100.36 & 2.307 & 1.647 & 0.024 & 0.741 & 0.024 & 0.229 & 0.000 & 4.972 & 76.4 & 49.9 \\
\hline $181 \mathrm{M}-1,115-124 \mathrm{~cm}$ & EL-4 & 50.96 & 30.47 & 0.865 & 14.84 & 0.286 & 2.82 & 0.013 & 100.25 & 2.318 & 1.634 & 0.030 & 0.724 & 0.019 & 0.249 & 0.001 & 4.974 & 74.4 & 39.6 \\
\hline $181 \mathrm{M}-1,115-124 \mathrm{~cm}$ & EL-4 & 51.02 & 30.11 & 0.918 & 14.57 & 0.256 & 2.97 & 0.006 & 99.84 & 2.330 & 1.621 & 0.032 & 0.713 & 0.017 & 0.263 & 0.000 & 4.976 & 73.1 & 35.6 \\
\hline $181 \mathrm{M}-1,115-124 \mathrm{~cm}$ & EL-4 & 50.88 & 29.98 & 0.883 & 14.18 & 0.282 & 3.07 & 0.000 & 99.27 & 2.334 & 1.622 & 0.031 & 0.697 & 0.019 & 0.273 & .000 & 4.976 & 71.9 & 38.7 \\
\hline $181 \mathrm{M}-1,115-124 \mathrm{~cm}$ & EL-5 & 51.20 & 30.17 & 1.120 & 14.46 & 0.186 & 3.04 & 0.005 & 100.18 & 2.331 & 1.619 & 0.038 & 0.705 & 0.013 & 0.268 & 0.000 & 4.975 & 72.4 & 24.7 \\
\hline $140-$ & & & & & & & & & & & & & & & & & & & \\
\hline $18-24 \mathrm{~cm}$ & EL-1 & 47.36 & 32.94 & 413 & 17.16 & 269 & 1.31 & 0.013 & 99.46 & 2.184 & 1.790 & 0.014 & 0.848 & 0.018 & 0.117 & 0.001 & 4.973 & 87.8 & 56.3 \\
\hline $200 \mathrm{R}-01,18-24 \mathrm{~cm}$ & EL-1 & 47.52 & 33.31 & 0.321 & 17.20 & .231 & 1.38 & 0.010 & 99.98 & 2.180 & 1.801 & 0.011 & 0.845 & 0.016 & 0.123 & 0.001 & 4.976 & 87.3 & 58.8 \\
\hline $18-24 \mathrm{~cm}$ & EL-1 & 45.99 & 32.75 & 0.415 & 17.15 & 0.215 & 1.53 & 0.000 & 98.05 & 2.158 & 1.811 & 0.015 & 0.862 & 0.015 & 0.139 & 0.000 & 4.999 & 86.1 & 50.7 \\
\hline $200 \mathrm{R}-1,18-24 \mathrm{~cm}$ & EL-1 & 47.05 & 32.76 & 0.416 & 17.13 & 0.225 & 1.52 & 0.001 & 99. & 2.180 & 1.789 & 0.014 & 0.851 & 0.016 & 0.136 & 0.000 & 4.986 & 86.2 & 51.7 \\
\hline $200 \mathrm{R}-1,18-24 \mathrm{~cm}$ & EL-1 & 46.73 & 33.06 & 0.468 & 17.63 & 0.161 & 1.10 & 0.000 & 99.14 & 2.165 & 1.806 & 0.016 & 0.876 & 0.011 & 0.098 & 0.000 & 4.973 & 89.9 & 40.5 \\
\hline $200 \mathrm{R}-1,18-24 \mathrm{~cm}$ & EL-2 & 49.68 & 31.06 & 0.872 & 14.46 & 0.255 & 3.02 & 0.006 & 99.36 & 2.283 & 1.683 & 0.030 & 0.712 & 0.017 & 0.269 & 0.000 & 4.995 & 72.5 & 36.7 \\
\hline$-24 \mathrm{~cm}$ & EL-3 & 50.65 & 30.35 & 0.700 & 14,42 & 0.272 & 3.06 & 0.015 & 99.47 & 2.321 & 1.639 & 0.024 & 0.708 & 0.019 & 0.272 & 0.001 & & 72.2 & 43.5 \\
\hline 200R-1, 1 & EL-3 & 50.47 & 31.91 & 0.804 & 15.05 & 0.343 & 2.83 & 0.006 & 101.42 & 2.273 & 1.694 & 0.027 & 0.726 & 0.023 & 0.247 & 0.000 & 4.990 & 74.6 & 45.8 \\
\hline $200 \mathrm{R}-1,18-24 \mathrm{~cm}$ & EL-3 & 50.11 & 31.02 & 0.747 & 14.90 & 0.310 & 2.84 & 0.008 & 99.93 & 2.289 & 1.671 & 0.026 & 0.729 & 0.021 & 0.252 & 0.000 & 4.988 & 74.3 & 45.1 \\
\hline$-24 \mathrm{~cm}$ & EL-4 & 50.56 & 30.77 & 0.540 & 15.14 & 0.277 & 2.71 & 0.000 & 99.99 & 2.306 & 1.654 & 0.019 & 0.740 & 0.019 & 0.240 & 0.000 & 4.977 & 75.5 & 50.4 \\
\hline $200 \mathrm{R}-1,18-24 \mathrm{~cm}$ & EL-4 & 50.19 & 30.43 & 0.589 & 15.51 & 0.305 & 2.38 & 0.007 & 99 . & & 47 & 0 & 0.763 & 0.021 & 0.212 & 0.000 & & 78.2 & 50.6 \\
\hline $200 \mathrm{R}-1,18-24 \mathrm{~cm}$ & $\mathrm{EL}-4$ & 49.49 & 30.04 & 0.509 & 15.37 & 0.320 & 2.40 & 0.000 & 98.13 & 2.303 & 1.648 & 0.018 & 0.766 & 0.022 & 0.217 & 0.000 & 4.973 & 78.0 & 55.5 \\
\hline$-24 \mathrm{~cm}$ & EL-4 & 49.53 & 30.22 & 0.559 & 15.29 & 0.298 & 2.44 & 0.021 & 98.36 & 2.299 & 1.654 & 0.020 & 0.761 & 0.021 & 0.220 & 0.001 & 4.975 & 77.5 & 51.4 \\
\hline $24 \mathrm{~cm}$ & EL-4 & 50.20 & 30.92 & 0.602 & 15.01 & 0.281 & 2.57 & 0.000 & 99.59 & 2.298 & 1.669 & 0.021 & 0.736 & 0.019 & & 0.000 & & & 48.0 \\
\hline$-24 \mathrm{~cm}$ & EL-5 & 50.19 & 30.59 & 0.632 & 15.31 & 0.241 & 2.54 & 0.011 & 99.51 & 2.302 & 1.654 & 0.022 & 0.752 & 0.016 & 0.226 & 0.001 & 4.973 & 76.9 & 43.0 \\
\hline $200 \mathrm{R}-1,18-24 \mathrm{~cm}$ & EL-5 & 50.18 & 31.14 & 0.437 & 15.21 & 0.318 & 2.60 & 0.017 & 99.90 & 2.291 & 1.676 & 0.015 & 0.744 & 0.022 & 0.2 & 0.001 & 4.979 & 76.3 & 59.0 \\
\hline$-24 \mathrm{~cm}$ & EL-5 & 50.20 & 29.87 & 0.500 & 15.14 & 0.305 & 2.60 & 0.003 & 98.61 & 2.322 & 1.629 & 0.017 & 0.750 & 0.021 & 0. & 0.000 & & $7 c$ & 54.7 \\
\hline$-24 \mathrm{~cm}$ & EL-5 & 50.44 & 29.09 & 0.590 & 14.85 & 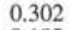 & & 0 & 98 & & & 0. & 0.7 & & & 0.000 & & & 50.3 \\
\hline $1,8-12 \mathrm{~cm}$ & EL-1 & 51.28 & 29.26 & 0.837 & 13.18 & 0.183 & 3.74 & 0.006 & 98 & 58 & 1.593 & 0.029 & 0.652 & 0. & 0. & 0.000 & 4.989 & 66.1 & 30.2 \\
\hline $226 \mathrm{R}-1,8$ & EL-1 & 52.59 & 28.85 & 0.961 & 13.56 & 0.180 & 3.81 & 0.000 & 99.96 & 2.393 & 1.548 & 0.033 & 0.661 & 0.012 & 0. & 0.000 & 4. & 66.3 & 27.1 \\
\hline $12 \mathrm{~cm}$ & EL-1 & 51.20 & 28.26 & 0.995 & 13.14 & 0.170 & 3.6 & 0.019 & 97. & 0 & 1.5 & $0 .($ & 0. & 0. & & 0.001 & & 8 & 25.3 \\
\hline , $8-12 \mathrm{~cm}$ & EL-1 & 51.10 & 28.32 & 1.040 & 12.80 & 0.194 & 3.70 & 0.009 & 97.17 & 0 & 1.561 & 0.0 & 0. & 0. & 0. & 0.001 & 4. & 6. & 27.0 \\
\hline$-1,8-12 \mathrm{~cm}$ & EL-2 & 48.96 & 30.83 & 0.619 & 14.65 & 0.281 & 2.74 & 0.000 & 98.08 & 2.278 & 1.691 & 0.022 & 0.731 & 0.1 & 0. & 0.000 & 4. & 74.7 & 47.3 \\
\hline $12 \mathrm{~cm}$ & EL-2 & 49.37 & 29.74 & 0.687 & 14.49 & 0.235 & 3.03 & 0.023 & 97.57 & 2.310 & 1.641 & 0.024 & 0.727 & 0. & & 0.001 & & 72 & 40.4 \\
\hline $2 \mathrm{~cm}$ & EL-3 & 51.30 & 29.61 & 0.715 & 15. & 2 & & 0 & 99 & & & & 0. & & & 0 & & 74.5 & 46.4 \\
\hline $\mathrm{cm}$ & EL-3 & 49.46 & 29.36 & 0.884 & 14.90 & 0.199 & 2.89 & 0.000 & 97. & & 1.620 & 0.031 & 0.747 & 0.014 & 0.262 & 0.000 & 4.990 & 74.0 & 30.8 \\
\hline $226 \mathrm{R}-1,8-12 \mathrm{~cm}$ & EL-3 & 50.61 & 29.22 & 0.859 & 13.98 & 0.176 & 3.02 & 0.006 & 97.87 & 2.354 & 1.602 & 0.030 & 0.697 & 0.012 & 0.272 & 0.000 & & 71.9 & 28.9 \\
\hline $2 \mathrm{~cm}$ & EL-3 & 49.99 & 29.70 & 0.976 & 14 & 0.2 & & & 98 & & & & & & & & & & 2 \\
\hline $\mathrm{cm}$ & EL-3 & 50.13 & 29.96 & 0.752 & 14.33 & 0.171 & 2. & 3 & 98. & 24 & 1.6 & 0. & 0.712 & & 0. & 0.000 & 4. & 72.8 & 31.1 \\
\hline $226 \mathrm{R}-1,8-12 \mathrm{~cm}$ & EL-3 & 50.26 & 29.73 & 0.837 & 14.02 & 0.237 & 3.18 & 0.011 & 98.28 & 2.331 & 1.625 & 0.029 & 0.697 & 0.016 & 0.286 & 0.001 & 4.985 & 70.8 & 35.9 \\
\hline $\begin{array}{r}\text { Elon } \\
13\end{array}$ & & & & & & & & & & & & & & & & & & & \\
\hline $115-124 \mathrm{~cm}$ & $-1 r$ & 0 & 91 & 945 & 14.10 & 277 & 4 & 000 & & 2.377 & .560 & 0.033 & 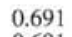 & 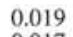 & 0. & 0. & 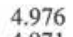 & 70 & 36.7 \\
\hline $4 \mathrm{~cm}$ & $-1 \mathrm{r}$ & 52.01 & 4 & 1 & 1 & 245 & & 3 & s & & & & 1 & & & 1 & 1 & & 33.3 \\
\hline $24 \mathrm{~cm}$ & EL-2r & 52.94 & 28.23 & 0.875 & 13.37 & 0.173 & 3.77 & 0.000 & 9 & .420 & 21 & 30 & 0.655 & 0.012 & 0. & 0.000 & 4.972 & 66.2 & 28.1 \\
\hline $181 \mathrm{M}-1,115-124 \mathrm{~cm}$ & EL-2r & 53.37 & 28.31 & 1.173 & 13.29 & 0.189 & 3.85 & 0.015 & 100.20 & 2.421 & 1.514 & 0.040 & 0.646 & 0.013 & 0. & 0.001 & 4.972 & 65.6 & 24.2 \\
\hline $124 \mathrm{~cm}$ & EL-3r & 53.30 & 29.03 & 1.081 & 13.03 & 0.193 & & 0.028 & & 2.4 & & & & & & & & & 26.1 \\
\hline $124 \mathrm{~cm}$ & EL-3r & 53.12 & 28.59 & 1.096 & 13.21 & 0.194 & 3 & 0.000 & 1( & 2412 & 1 & 0.0 & 0. & 0. & 0. & 0.000 & 4. & 65.7 & 26.0 \\
\hline M-1, $115-124 \mathrm{~cm}$ & EL-4r & 51.78 & 29.45 & 0.883 & 13.98 & 0.233 & 3.2 & 0.010 & 99.59 & 2.365 & 1.586 & 0.030 & 0.684 & 0.016 & 0.289 & 0.001 & 4.971 & 70.3 & 34.3 \\
\hline $181 \mathrm{M}-1,115-124 \mathrm{~cm}$ & EL-5r & 52.14 & 29.77 & 1.180 & 14.03 & 0.152 & 3.36 & 0.000 & 100.64 & 2.360 & 1.588 & 0.040 & 0.681 & 0.010 & 0.295 & 0.000 & 4.974 & 69.8 & 20.3 \\
\hline & & & & & & & & & & & & & & & & & & & \\
\hline $\mathrm{cm}$ & EL-1r & .83 & 58 & 08 & & 64 & 0 & 5 & 99.13 & 99 & 94 & 8 & & 8 & & & & 71 & 9.3 \\
\hline $\mathrm{cm}$ & $-1 \mathrm{r}$ & 52.17 & 2 & 3 & & $=$ & & & & & & & & & & & & & 4 \\
\hline $200 \mathrm{R}-1,18-24 \mathrm{~cm}$ & EL-2r & 56.29 & 25.61 & 0.941 & 9. & & 5.84 & 0.6 & 97. & & & 32 & 0.4 & & & 0.001 & 4.971 & 46.3 & 12.8 \\
\hline $200 \mathrm{R}-1,18-24 \mathrm{~cm}$ & EL-2r & 54.14 & 26.59 & 1.096 & 10.64 & 0.114 & 5.24 & 0.037 & 97.86 & 2.501 & 1.448 & 0.038 & 0.527 & 0.008 & 0.469 & 0.002 & 4.992 & 52.8 & 17.1 \\
\hline$-1,18-24 \mathrm{~cm}$ & $E L-2 r$ & 52.36 & 28.27 & 1.016 & 11.97 & 0.196 & 4.57 & 0.000 & 98.39 & 2.416 & 1.538 & 0.035 & 0.5 & & & & & & 27.6 \\
\hline$-24 \mathrm{~cm}$ & EL-2r & 52.53 & 29.65 & 0.915 & 12,70 & 0213 & 40 & 0.012 & 100.11 & 7 & 5 & 0.031 & 0.617 & 0. & 0. & 0.001 & 0 & 63.1 & 31.6 \\
\hline $200 \mathrm{R}-1,18-24 \mathrm{~cm}$ & EL-2r & 54.28 & & 1.290 & 11 & 0.118 & 5.1 & 0.022 & & & 9 & 0.0 & 0.542 & 0.008 & 0.462 & 0.001 & 4.995 & 53.9 & 15.3 \\
\hline $200 \mathrm{R}-1,18-24 \mathrm{~cm}$ & EL-3r & 55.31 & 26.48 & 1.084 & 11.29 & 0.149 & 4.93 & 0.005 & 99.25 & 2.517 & 1.421 & 0.037 & 0.551 & 0.010 & 0.435 & 0.000 & 4.971 & 55.8 & 21.4 \\
\hline $\mathrm{R}-1,18-24 \mathrm{~cm}$ & EL-3r & 53.74 & 27.83 & 0.917 & 13.09 & 0.243 & & 0.004 & 99.64 & 2.445 & 1.493 & 0.031 & 0.638 & & & 0.000 & 4.961 & 65.4 & 34.4 \\
\hline $4 \mathrm{~cm}$ & EL-3r & 57.30 & 25.75 & 0.907 & $9 .($ & & & 0.040 & 98. & & & & & & & $0 .($ & 4. & 46.0 & 8.0 \\
\hline $200 \mathrm{R}-1,18-24 \mathrm{~cm}$ & EL-3r & 60.29 & 25.24 & 1.067 & 8.12 & 0.043 & 6.55 & 0.032 & 101.33 & 2.657 & 1.311 & 0.035 & 0.383 & 0.003 & 0.560 & 0.002 & 4.951 & 40.6 & 7.4 \\
\hline $200 \mathrm{R}-1,18-24 \mathrm{~cm}$ & $\mathrm{EL}-4 \mathrm{r}$ & 54.09 & 28.10 & 0.891 & 12.46 & 0.190 & 3.95 & 0.017 & 99.70 & 2.454 & 1.503 & 0.030 & 0.606 & 0.013 & & 0.001 & 4.954 & 63.5 & 29.7 \\
\hline $\mathrm{R}-1,18-24 \mathrm{~cm}$ & EL-4r & 52.95 & 28.29 & 0.854 & 13.17 & 0.235 & 3.76 & 0.000 & 99.27 & 2.421 & 1.525 & 0.029 & 0.645 & & & 0.000 & & 65.9 & 35.3 \\
\hline $24 \mathrm{~cm}$ & EL-4r & 54.93 & & 1.187 & 11.34 & & 4. & 0.0 & & & & & & & & 0.001 & & 56.2 & 17.7 \\
\hline $200 \mathrm{R}-1,18-24 \mathrm{~cm}$ & EL-4r & 56.82 & 26.10 & 1.111 & 10.54 & 0.089 & 5.58 & 0.008 & 100.25 & 2.555 & 1.384 & 0.038 & 0.508 & 0.006 & 0.487 & 0.000 & 4.978 & 51.0 & 13.7 \\
\hline $200 \mathrm{R}-1,18-24 \mathrm{~cm}$ & EL-5r & 53.08 & 29.76 & 0.907 & 13.60 & 0.204 & 3.69 & 0.000 & 101.23 & 2.382 & 1.575 & 0.031 & 0.654 & 0.014 & 0.321 & 0.000 & 4.976 & 67.1 & 30.8 \\
\hline $\mathrm{R}-1,18-24 \mathrm{~cm}$ & EL-5r & 50.99 & 29.59 & 0.765 & 14.36 & 0.183 & 3.36 & 0.001 & 99.25 & 2.343 & 1.603 & & & & & 0.000 & 4.992 & 70.2 & 32.1 \\
\hline$-24 \mathrm{~cm}$ & EL-5r & 51.66 & 30.08 & 0.727 & 14.49 & & $3 .($ & 0.014 & & & & & 0.7 & & & 0.001 & 4.974 & 72.2 & 45.5 \\
\hline $200 \mathrm{R}-1,18-24 \mathrm{~cm}$ & EL-5r & 52.61 & 28.69 & 0.858 & 13.46 & 0.247 & 3.63 & 0.017 & 99.52 & 2.402 & 1.544 & 0.029 & 0.658 & 0.017 & 0.321 & 0.001 & 4.973 & 67.1 & 36.3 \\
\hline $1,18-24 \mathrm{~cm}$ & EL-5r & 53.34 & 27.44 & 0.978 & 12.07 & 0.196 & 4.11 & 0.027 & 98.16 & 2.459 & 1.491 & 0.034 & 0.596 & 0.013 & 0.367 & 0.002 & 4.963 & 61.8 & 28.4 \\
\hline $200 \mathrm{R}-1,18-24 \mathrm{~cm}$ & EL-5r & 54.40 & 26.38 & 1.042 & 11.24 & 0.136 & 4.74 & 0.009 & 97.94 & 2.509 & 1.434 & 0.036 & 0.555 & 0.009 & 0.424 & 0.001 & 4.968 & 56.7 & 20.5 \\
\hline
\end{tabular}

ophiolitic pyroxenes (Loucks, 1990). On the multivariate plot of Koloskov and Zharinov (1993) most analyses fall within the field for mid-ocean-ridge pyroxenes (Fig. 5). All four pyroxene types follow the same compositional curves in terms of $\mathrm{Cr}$ vs. Mg\# (Fig. 6), Mn vs. $\mathrm{Mg \#} \mathrm{(Fig.} \mathrm{7),} \mathrm{and} \mathrm{Ca} \mathrm{vs.} \mathrm{Mg \#} \mathrm{(Fig.} \mathrm{8).} \mathrm{The} \mathrm{highest} \mathrm{Cr}$ contents occur in phenocryst cores, but the cores of some microphenocrysts and poikilitic pyroxenes have significant $\mathrm{Cr}_{2} \mathrm{O}_{3}$ contents $(0.25 \%-0.50 \%)$ indicating that they nucleated and began to grow before the surrounding magma was depleted in $\mathrm{Cr}_{2} \mathrm{O}_{3}$.

Olivine phenocrysts were analyzed in two samples (Table 11), and are plotted along with the pyroxenes on Figures 6, 7, and 8. Core compositions range from $\mathrm{Mg} \# 84$ to $\mathrm{Mg} \# 88$. In most grains the outer part 
Table 5. Skeletal plagioclase.

\begin{tabular}{|c|c|c|c|c|c|c|c|c|c|c|c|c|c|c|c|c|c|c|c|}
\hline \multirow[b]{2}{*}{ Sample } & \multirow[b]{2}{*}{ Grain } & \multicolumn{8}{|c|}{ Weight percent } & \multicolumn{10}{|c|}{ Cations per 8 oxygens } \\
\hline & & $\mathrm{SiO}_{2}$ & $\mathrm{Al}_{2} \mathrm{O}_{3}$ & $\mathrm{Fe}_{2} \mathrm{O}_{3}$ & $\mathrm{CaO}$ & $\mathrm{MgO}$ & $\mathrm{Na}_{2} \mathrm{O}$ & $\mathrm{K}_{2} \mathrm{O}$ & Total & $\mathrm{Si}$ & Al & $\mathrm{Fe}$ & $\mathrm{Ca}$ & $\mathrm{Mg}$ & $\mathrm{Na}$ & K & Total & An\% & Mg\# \\
\hline \multicolumn{20}{|l|}{$\begin{array}{l}\text { Skeletal plagioclase cores } \\
\text { 137-504B- }\end{array}$} \\
\hline $181 \mathrm{M}-1,115-124 \mathrm{~cm}$ & SK-1 & 57.27 & 25.99 & 1.110 & 10.06 & 0.064 & 5.83 & 0.026 & 100.35 & 2.570 & 1.375 & 0.038 & 0.484 & 0.004 & 0.507 & 0.002 & 4.979 & 48.7 & 10.2 \\
\hline $181 \mathrm{M}-1,115-124 \mathrm{~cm}$ & SK-1 & 57.39 & 25.94 & 1.014 & 9.75 & 0.050 & 5.97 & 0.005 & 100.12 & 2.578 & 1.374 & 0.034 & 0.469 & 0.003 & 0.520 & 0.000 & 4.978 & 47.4 & 8.9 \\
\hline $181 \mathrm{M}-1,115-124 \mathrm{~cm}$ & SK-1 & 57.06 & 25.93 & 1.171 & 10.13 & 0.050 & 5.94 & 0.034 & 100.32 & 2.564 & 1.374 & 0.040 & 0.488 & 0.003 & 0.518 & 0.002 & 4.989 & 48.4 & 7.8 \\
\hline $181 \mathrm{M}-1,115-124 \mathrm{~cm}$ & SK-1 & 57.45 & 26.11 & 0.946 & 9.77 & 0.060 & 6.01 & 0.031 & 100.37 & 2.574 & 1.379 & 0.032 & 0.469 & 0.004 & 0.522 & 0.002 & 4.982 & 47.3 & 11.2 \\
\hline $181 \mathrm{M}-1,115-124 \mathrm{~cm}$ & SK-2 & 58.70 & 26.36 & 1.157 & 9.48 & 0.035 & 5.81 & 0.020 & 101.56 & 2.592 & 1.372 & 0.038 & 0.449 & 0.002 & 0.498 & 0.001 & 4.952 & 47.4 & 5.7 \\
\hline $181 \mathrm{M}-1,115-124 \mathrm{~cm}$ & SK-2 & 57.34 & 27.32 & 1.064 & 10.41 & 0.076 & 5.33 & 0.017 & 101.56 & 2.539 & 1.426 & 0.036 & 0.494 & 0.005 & 0.458 & 0.001 & 4.959 & 51.8 & 12.4 \\
\hline $181 \mathrm{M}-1,115-124 \mathrm{~cm}$ & SK-2 & 58.76 & 26.32 & 1.160 & 9.38 & 0.036 & 5.76 & 0.026 & 101.44 & 2.596 & 1.371 & 0.039 & 0.444 & 0.002 & 0.493 & 0.002 & 4.947 & 47.3 & 5.8 \\
\hline $181 \mathrm{M}-1,115-124 \mathrm{~cm}$ & SK-3 & 51.21 & 29.35 & 0.813 & 14.31 & 0.218 & 3.06 & 0.004 & 98.96 & 2.356 & 1.592 & 0.028 & 0.706 & 0.015 & 0.273 & 0.000 & 4.970 & 72.1 & 34.7 \\
\hline $181 \mathrm{M}-1,115-124 \mathrm{~cm}$ & SK-4 & 51.71 & 29.89 & 1.023 & 14.21 & 0.148 & 3.15 & 0.014 & 100.14 & 2.351 & 1.602 & 0.035 & 0.692 & 0.010 & 0.278 & 0.001 & 4.969 & 71.3 & 22.3 \\
\hline $181 \mathrm{M}-1,115-124 \mathrm{~cm}$ & SK-4 & 50.53 & 30.61 & 0.763 & 14.90 & 0.286 & 2.69 & 0.006 & 99.78 & 2.309 & 1.649 & 0.026 & 0.730 & 0.020 & 0.238 & 0.000 & 4.972 & 75.4 & 42.6 \\
\hline $181 \mathrm{M}-1,115-124 \mathrm{~cm}$ & SK-4 & 51.09 & 30.78 & 0.853 & 14.73 & 0.276 & 2.83 & 0.009 & 100.56 & 2.316 & 1.645 & 0.029 & 0.715 & 0.019 & 0.248 & 0.001 & 4.972 & 74.2 & 39.1 \\
\hline $181 \mathrm{M}-1,115-124 \mathrm{~cm}$ & SK-4 & 51.03 & 30.42 & 1.073 & 14.42 & 0.189 & 2.89 & 0.000 & 100.02 & 2.325 & 1.634 & 0.037 & 0.704 & 0.013 & 0.255 & 0.000 & 4.967 & 73.4 & 25.9 \\
\hline $181 \mathrm{M}-1,115-124 \mathrm{~cm}$ & SK-5 & 51.67 & 29.68 & 0.765 & 14.62 & 0.262 & 2.90 & 0.004 & 99.89 & 2.355 & 1.595 & 0.026 & 0.714 & 0.018 & 0.256 & 0.000 & 4.963 & 73.6 & 40.4 \\
\hline $\begin{array}{l}181 \mathrm{M}-1,115-124 \mathrm{~cm} \\
140-504 \mathrm{~B}-\end{array}$ & SK-5 & 51.20 & 29.61 & 0.968 & 14.38 & 0.217 & 3.14 & 0.000 & 99.51 & 2.346 & 1.599 & 0.033 & 0.706 & 0.015 & 0.279 & 0.000 & 4.977 & 71.7 & 30.7 \\
\hline $226 \mathrm{R}-1,8-12 \mathrm{~cm}$ & SK-1 & 49.40 & 30.96 & 0.722 & 15.02 & 0.240 & 2.77 & 0.002 & 0 & 2.278 & 1.683 & 0.025 & 0.742 & 0.016 & 0.247 & 0.000 & 4.992 & 75.0 & 39.7 \\
\hline $226 \mathrm{R}-1,8-12 \mathrm{~cm}$ & SK-1 & 49.62 & 30.07 & 0.534 & 14.58 & 0.313 & 2.95 & 0.000 & 98.07 & 2.308 & 1.649 & 0.019 & 0.727 & 0.022 & 0.266 & 0.000 & 4.990 & 73.2 & 53.7 \\
\hline $226 \mathrm{R}-1,8-12 \mathrm{~cm}$ & SK-1 & 49.33 & 30.62 & 0.548 & 14.65 & 0.274 & 2.68 & 0.003 & 98.10 & 2.293 & 1.678 & 0.019 & 0.730 & 0.019 & 0.241 & 0.000 & 4.980 & 75.1 & 49.8 \\
\hline $226 \mathrm{R}-1,8-12 \mathrm{~cm}$ & SK-2 & 48.38 & 30.77 & 0.573 & 14.97 & 0.309 & 2.60 & 0.004 & 97.61 & 2.265 & 1.699 & 0.020 & 0.751 & 0.022 & 0.236 & 0.000 & 4.993 & 76.1 & 51.6 \\
\hline $226 \mathrm{R}-1,8-12 \mathrm{~cm}$ & SK-2 & 49.82 & 29.61 & 0.784 & 13.64 & 0.187 & 3.45 & 0.000 & 97.50 & 2.329 & 1.632 & 0.028 & 0.683 & 0.013 & 0.313 & 0.000 & 4.998 & 68.6 & 32.1 \\
\hline$-1,8-12 \mathrm{~cm}$ & SK-2 & 50.60 & 29.45 & 0.731 & 13.07 & 0.216 & 3.79 & 0.007 & 97.85 & 2.352 & 1.614 & 0.026 & 0.651 & 0.015 & 0.341 & 0.000 & 4.999 & 65.6 & 36.9 \\
\hline$-1,8-12 \mathrm{~cm}$ & SK-3 & 49.81 & 29.16 & 1.234 & 12.82 & 0.183 & 3.93 & 0.019 & 97.15 & 2.339 & 1.614 & 0.044 & 0.645 & 0.0 & 0.357 & 0.001 & 5.012 & 64.3 & 22.7 \\
\hline $226 \mathrm{R}-1,8-12 \mathrm{~cm}$ & SK-3 & 50.54 & 29.05 & 0.962 & 12.83 & 0.160 & 3.87 & 0.009 & 97.42 & 2.361 & 1.600 & 0.034 & 0.642 & 0.011 & 0.350 & 0.001 & 4.998 & 64.7 & 24.8 \\
\hline \multicolumn{20}{|l|}{$\begin{array}{l}\text { Skeletal plagioclase rims } \\
\text { 137-504B- }\end{array}$} \\
\hline $181 \mathrm{M}-1,115-124 \mathrm{~cm}$ & SK-1r & 58.82 & 25.81 & 0.789 & 9.08 & 0.008 & 6.17 & 0.036 & 100.71 & 2.616 & 1.353 & 0.026 & 0.433 & 0.001 & 0.532 & 0.002 & 4.962 & 44.8 & 2.0 \\
\hline $181 \mathrm{M}-1,115-124 \mathrm{~cm}$ & SK-5r & 52.42 & 28.42 & 1.059 & 13.41 & 0.200 & 3.84 & 0.000 & 99.35 & 2.401 & 1.535 & 0.037 & 0.658 & 0.014 & 0.341 & 0.000 & 4.984 & 65.9 & 27.2 \\
\hline $\begin{array}{l}140-504 \mathrm{~B}- \\
226 \mathrm{R}-1,8-12 \mathrm{~cm}\end{array}$ & & & & & & & & & & & & & & & & & & & \\
\hline $\begin{array}{l}220 \mathrm{~K}-1,8-12 \mathrm{~cm} \\
226 \mathrm{R}-1,8-12 \mathrm{~cm}\end{array}$ & SK-Ir & $\begin{array}{l}49.74 \\
49.51\end{array}$ & $\begin{array}{l}30.05 \\
29.53\end{array}$ & 0.734 & $\begin{array}{l}14.68 \\
13.05\end{array}$ & $\begin{array}{l}0.251 \\
0.262\end{array}$ & 3.11 & 0.000 & 98.57 & 2.306 & 1.642 & 0.026 & 0.729 & 0.017 & 0.280 & 0.000 & 5.000 & 72.3 & 40.3 \\
\hline $226 \mathrm{R}-1,8-12 \mathrm{~cm}$ & SK-2r & 58.50 & 23.92 & 0.824 & $\begin{array}{l}13.95 \\
6.67\end{array}$ & $\begin{array}{l}0.202 \\
0.005\end{array}$ & $\begin{array}{l}3.23 \\
7.64\end{array}$ & $\begin{array}{l}0.002 \\
0.076\end{array}$ & $\begin{array}{l}91.16 \\
97.62\end{array}$ & $\begin{array}{l}2.323 \\
2.677\end{array}$ & $\begin{array}{l}1.034 \\
1.290\end{array}$ & $\begin{array}{l}0.024 \\
0.028\end{array}$ & $\begin{array}{l}0.102 \\
0.327\end{array}$ & 0.000 & $\begin{array}{l}0.294 \\
0.678\end{array}$ & 0.004 & $\begin{array}{l}4.995 \\
5.005\end{array}$ & $\begin{array}{l}70.4 \\
32.4\end{array}$ & $\begin{array}{r}43.0 \\
1.2\end{array}$ \\
\hline $226 \mathrm{R}-1,8-12 \mathrm{~cm}$ & SK-2r & 56.38 & 25.53 & 0.874 & 8.43 & 0.081 & 6.54 & 0.015 & 97.84 & 2.586 & 1.381 & 0.030 & 0.414 & 0.006 & 0.581 & 0.001 & 4.999 & 41.6 & 15.5 \\
\hline $226 \mathrm{R}-1,8-12 \mathrm{~cm}$ & SK-2r & 52.76 & 27.81 & 1.198 & 11.20 & 0.118 & 4.78 & 0.008 & 97.88 & 2.442 & 1.517 & 0.042 & 0.555 & 0.008 & 0.429 & 0.000 & 4.993 & 56.4 & 16.3 \\
\hline $226 \mathrm{R}-1,8-12 \mathrm{~cm}$ & SK-3r & 56.39 & 25.59 & 0.870 & 8.34 & 0.052 & 6.65 & 0.046 & 97.93 & 2.585 & 1.383 & 0.030 & 0.410 & 0.004 & 0.591 & 0.003 & 5.005 & 40.8 & 10.6 \\
\hline $226 \mathrm{R}-1,8-12 \mathrm{~cm}$ & SK-3r & 53.11 & 28.04 & 1.182 & 12.23 & 0.119 & 4.54 & 0.019 & 99.23 & 2.430 & 1.513 & 0.041 & 0.600 & 0.008 & 0.402 & 0.001 & 4.995 & 59.8 & 16.6 \\
\hline
\end{tabular}
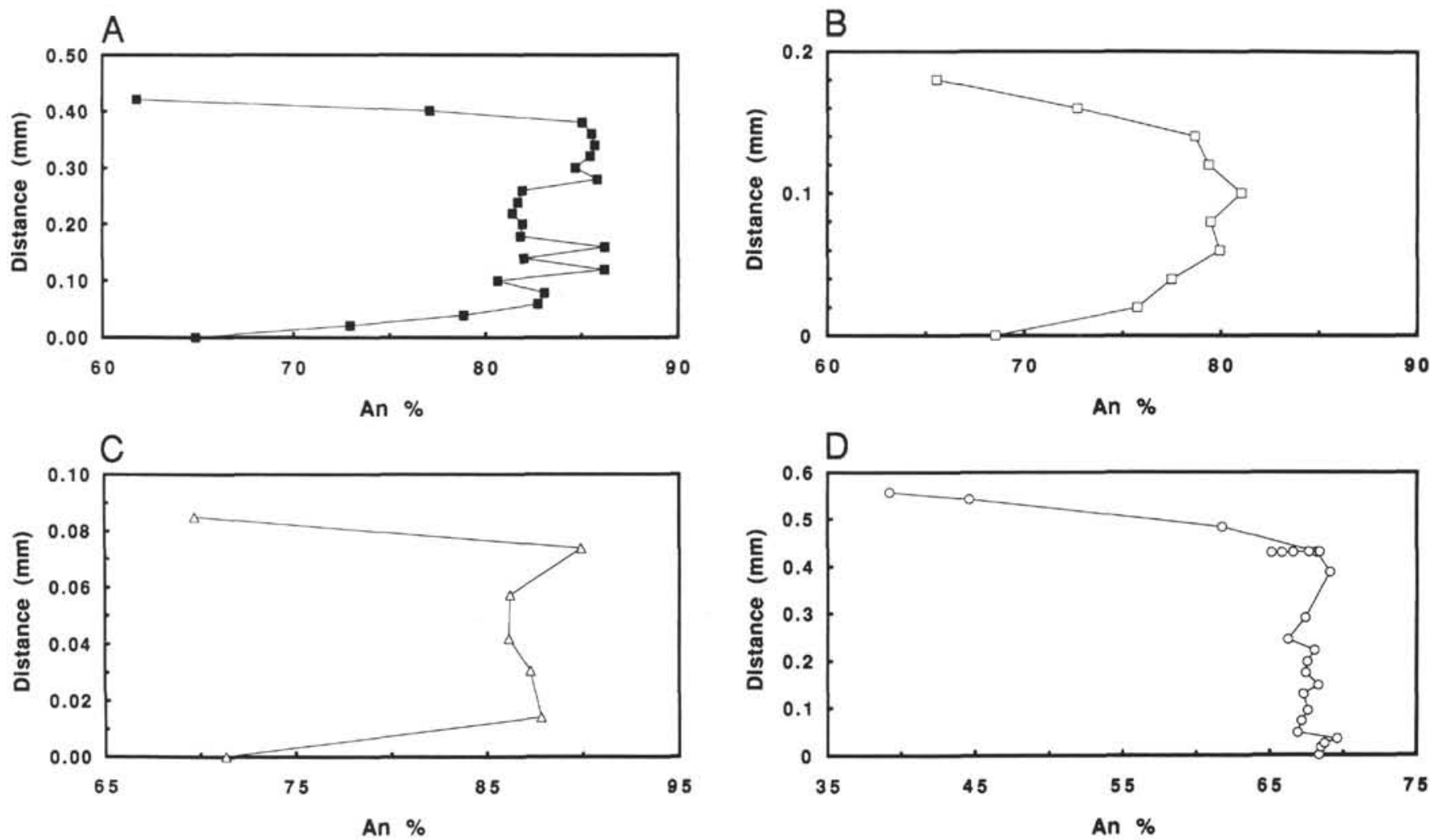

Figure 3. Zoning profiles in plagioclase crystals in Sample 140-504B-200R-1, 18-24 cm. A. Profile across the width of a plagioclase phenocryst. B. Profile across the width of a plagioclase microphenocryst. C. Profile across the width of an elongate plagioclase crystal. D. Profile along the length (and across the width at $0.43 \mathrm{~mm}$ ) of an individual plagioclase lath in a radial cluster. 

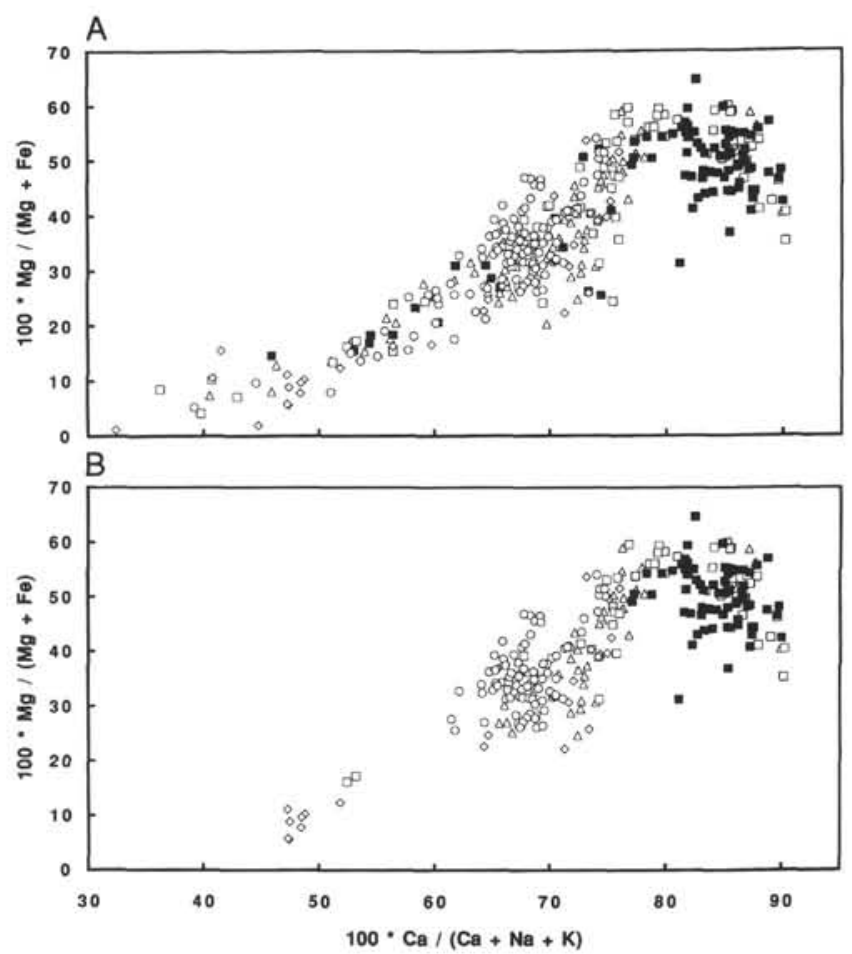

Figure 4. An\% vs. Mg\# for analyzed plagioclases in medium-grained diabase from Hole 504B. A. Core and rim compositions for plagioclase phenocrysts (filled squares); plagioclase microphenocrysts (open squares); elongate crystals (open triangles); skeletal crystals (open diamonds); and plagioclase radial clusters (open circles). B. Core compositions only, symbols as in (A).

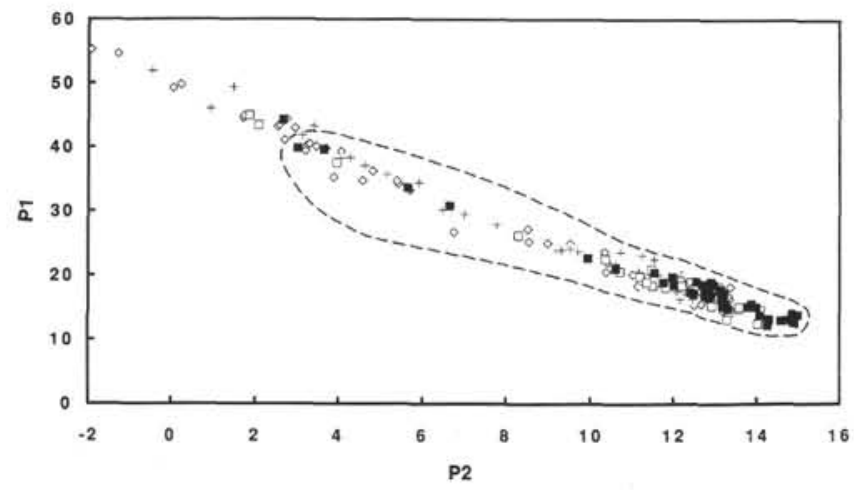

Figure 5. $\mathrm{P} 1$ vs. $\mathrm{P} 2$ multivariate plot for analyzed pyroxenes in medium-grained diabase from Hole 504B, where $\mathrm{Pl}=8-0.02 \mathrm{SiO}_{2}+0.09 \mathrm{TiO}_{2}+0.64 \mathrm{Al}_{2} \mathrm{O}_{3}$ $+0.59 \mathrm{Cr}_{2} \mathrm{O}_{3}+1.35 \mathrm{FeO}+9.65 \mathrm{MnO}-0.50 \mathrm{MgO}+0.29 \mathrm{CaO}-2.26 \mathrm{Na}_{2} \mathrm{O}$, and $\mathrm{P} 2=8-0.93 \mathrm{TiO}_{2}+0.07 \mathrm{Al}_{2} \mathrm{O}_{3}+1.23 \mathrm{Cr}_{2} \mathrm{O}_{3}-0.46 \mathrm{FeO}+1.74 \mathrm{MnO}+$ $0.36 \mathrm{MgO}+0.10 \mathrm{CaO}-1.66 \mathrm{Na}_{2} \mathrm{O}$ (Koloskov and Zharinov, 1993). Samples fall in the field for mid-ocean-ridge and island-arc pyroxenes (dashed line). Symbols: pyroxene phenocrysts (filled squares); pyroxene microphenocrysts (open squares); ophitic pyroxene (crosses); poikilitic pyroxene (open diamonds).

is altered, so the original rim composition cannot be determined. One unaltered rim had a composition of $\mathrm{Mg} \# 70$.

\section{DISCUSSION}

The order of crystallization of plagioclase crystal types can be determined from the compositional data. Plagioclase phenocrysts were the initial liquidus phase, followed by plagioclase microphenocrysts, elongate to skeletal crystals, and finally plagioclase radial

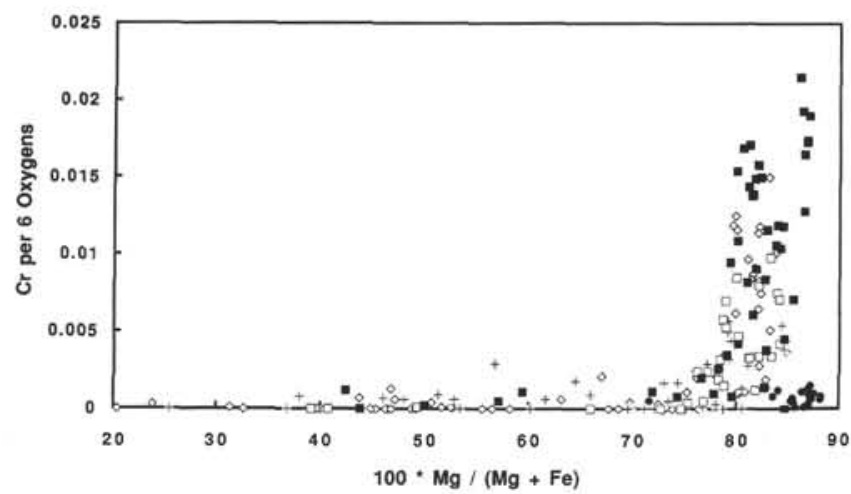

Figure 6. $\mathrm{Cr}$ vs. $\mathrm{Mg} \#$ for analyzed olivines and pyroxenes in medium-grained diabase from Hole 504B. Symbols as in Figure 5; olivines (filled circles).

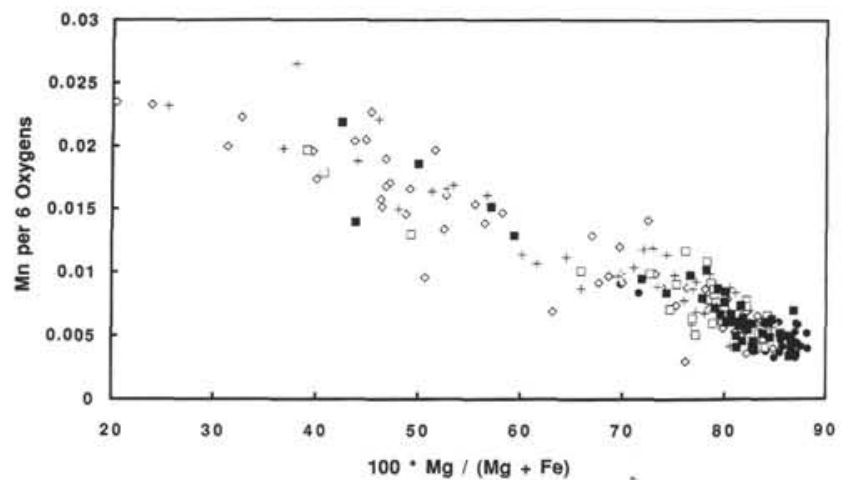

Figure 7. Mn vs. Mg\# for analyzed olivines and pyroxenes in medium-grained diabase from Hole 504B. Symbols as in Figure 6.



Figure 8. Ca vs. $\mathrm{Mg} \#$ for analyzed olivines and pyroxenes in medium-grained diabase from Hole 504B. Symbols as in Figure 6.

clusters. The earlier formed crystals continued to grow as each successive stage was nucleated so that the zoning on the outer part of the earlier crystals is compositionally similar to the cores of later crystals. The one exception to this general relationship is the radial clusters, which are unzoned except at their tips. They appear to have grown simultaneously with their enclosing poikilitic pyroxene so that individual radial clusters were armored with pyroxene as they grew. It is likely that plagioclase crystallization depleted the surrounding mag$\mathrm{ma}$ in plagioclase components and enriched it in pyroxene components, so that the rapid growth of the radial plagioclase crystals initiated pyroxene growth in the adjacent melt. Pyroxene growth thus kept pace with plagioclase growth, resulting in a texture in which a single optically continuous pyroxene crystal encloses a cluster or splay of radial plagioclase crystals. The margins of the resulting pyroxene out- 
Table 6. Plagioclase radial clusters.

Weight percent

Sample Grain $\begin{array}{llllllll}\mathrm{SiO}_{2} & \mathrm{Al}_{2} \mathrm{O}_{3} & \mathrm{Fe}_{2} \mathrm{O}_{3} & \mathrm{CaO} & \mathrm{MgO} & \mathrm{Na}_{2} \mathrm{O} & \mathrm{K}_{2} \mathrm{O} & \text { Total }\end{array}$

Cations per 8 oxygens

Radial plagioclase splays

$\begin{array}{llllllllll}200 \mathrm{R}-1,18-24 \mathrm{~cm} & \text { SP-1 } & 52.24 & 29.25 & 1.048 & 13.62 & 0.465 & 3.48 & 0.000 & 100.11\end{array}$ $\begin{array}{llllllllll}200 \mathrm{R}-1,18-24 \mathrm{~cm} & \text { SP-1 } & 52.64 & 29.35 & 0.968 & 13.83 & 0.250 & 3.51 & 0.006 & 100.55 \\ 2 & \text { SP-1 } & 52.04 & 28.03 & 0.968 & 13.80 & 0.411 & 3.46 & 0.014 & 98.72\end{array}$ $\begin{array}{llllllllll}200 \mathrm{R}-1,18-24 \mathrm{~cm} & \text { SP-1 } & 52.04 & 28.03 & 0.968 & 13.80 & 0.411 & 3.46 & 0.014 & 98.72 \\ 200 \mathrm{R}-1,18-24 \mathrm{~cm} & \text { SP-1 } & 52.04 & 29.50 & 0.926 & 13.95 & 0.194 & 3.37 & 0.007 & 99.99\end{array}$ $\begin{array}{llllllllll}200 \mathrm{R}-1,18-24 \mathrm{~cm} & \text { SP-1 } & 52.74 & 29.18 & 0.852 & 13.64 & 0.209 & 3.72 & 0.020 & 100.36\end{array}$ $\begin{array}{llllllllll}200 \mathrm{R}-1,18-24 \mathrm{~cm} & \text { SP-1 } & 52.75 & 29.18 & 0.852 & 13.64 & 0.209 & 3.72 & 0.020 & 100.36 \\ & 29.84 & 0.957 & 13.80 & 0.248 & 3.71 & 0.024 & 102.03\end{array}$ $\begin{array}{llllllllll}200 \mathrm{R}-1,18-24 \mathrm{~cm} & \text { SP-1 } & 53.45 & 29.84 & 0.957 & 13.80 & 0.248 & 3.71 & 0.024 & 102.03 \\ 200 \mathrm{~S}-1,18-24 & 52.95 & 29.82 & 0.987 & 13.73 & 0.345 & 3.63 & 0.016 & 101.47\end{array}$ $\begin{array}{llllllllll}200 \mathrm{R}-1,18-24 \mathrm{~cm} & \text { SP-1 } & 53.26 & 29.73 & 0.898 & 13.84 & 0.278 & 3.72 & 0.005 & 101.72\end{array}$

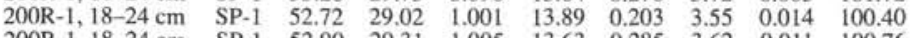
$\begin{array}{llllllllll}200 \mathrm{R}-1,18-24 \mathrm{~cm} & \text { SP-1 } & 52.90 & 29.31 & 1.005 & 13.63 & 0.285 & 3.62 & 0.011 & 100.76 \\ 200 \mathrm{R}-1,18-24 \mathrm{~cm} & \text { SP-1 } & 55.81 & 30.26 & 1.29 & 13.61 & 0.206 & 3.60 & 0.016 & 101.63\end{array}$

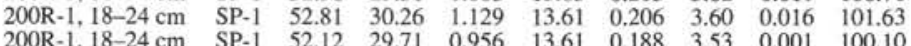
$\begin{array}{llllllllll}200 \mathrm{R}-1,18-24 \mathrm{~cm} & \text { SP-1 } & 53.04 & 29.15 & 0.955 & 13.69 & 0.221 & 3.85 & 0.010 & 100.91\end{array}$ $\begin{array}{llllllllll}200 \mathrm{R}-1,18-24 \mathrm{~cm} & \text { SP-1 } & 52.48 & 28.89 & 0.892 & 13.44 & 0.211 & 3.59 & 0.000 & 99.50\end{array}$ $\begin{array}{llllllllll}200 \mathrm{R}-1,18-24 \mathrm{~cm} & \text { SP- } & 52.48 & 28.89 & 0.892 & 13.44 & 0.211 & 3.59 & 0.000 & 99.50 \\ 200-1,18 \mathrm{~cm} & \text { SP-1 } & 51.96 & 29.64 & 0.820 & 13.64 & 0.207 & 3.37 & 0.009 & 99.65\end{array}$ $\begin{array}{lllllllllll}200 \mathrm{R}-1,18-24 \mathrm{~cm} & \text { SP-1 } & 52.65 & 28.73 & 0.836 & 13.29 & 0.240 & 3.43 & 0.000 & 99.18\end{array}$ $\begin{array}{llllllllll}200 \mathrm{R}-1,18-24 \mathrm{~cm} & \text { SP-1 } & 53.60 & 27.51 & 0.899 & 12.27 & 0.157 & 4.19 & 0.010 & 98.63\end{array}$ \begin{tabular}{llllllllll}
$200 \mathrm{R}-1,18-24 \mathrm{~cm}$ & SP-1 & 52.65 & 28.77 & 0.842 & 13.67 & 0.371 & 3.35 & 0.010 & 99.66 \\
\hline
\end{tabular} \begin{tabular}{lllllllllr}
$200 \mathrm{R}-1,18-24 \mathrm{~cm}$ & SP-1 & 52.64 & 28.46 & 0.959 & 13.61 & 0.252 & 3.47 & 0.010 & 99.41 \\
\hline
\end{tabular} $\begin{array}{lllllllllll}200 \mathrm{R}-1,18-24 \mathrm{~cm} & \text { SP-1 } & 53.28 & 28.75 & 0.822 & 13.35 & 0.227 & 3.70 & 0.013 & 100.13\end{array}$ $\begin{array}{llllllllll}200 \mathrm{R}-1,18-24 \mathrm{~cm} & \text { SP-1 } & 53.65 & 28.87 & 0.903 & 13.37 & 0.239 & 3.83 & 0.010 & 100.88\end{array}$ $\begin{array}{lllllllllr}200 \mathrm{R}-1,18-24 \mathrm{~cm} & \text { SP-1 } & 53.65 & 28.87 & 0.903 & 13.37 & 0.239 & 3.83 & 0.010 & 100.88 \\ 200 \mathrm{R}-1,18-24 \mathrm{~cm} & \text { SP-1 } & 53.36 & 28.65 & 0.789 & 13.10 & 0.196 & 3.87 & 0.027 & 99.98\end{array}$ $\begin{array}{llllllllll}200 \mathrm{R}-1,18-24 \mathrm{~cm} & \text { SP-2 } & 53.36 & 28.61 & 0.987 & 12.30 & 0.191 & 4.25 & 0.021 & 99.71\end{array}$

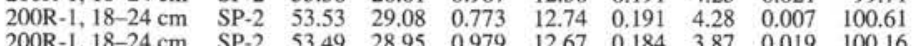
$\begin{array}{llllllllll}200 \mathrm{R}-1,18-24 \mathrm{~cm} & \text { SP-2 } & 53.49 & 28.95 & 0.979 & 12.67 & 0.184 & 3.87 & 0.019 & 100.16 \\ 200 \mathrm{R}-1,18-24 \mathrm{~cm} & \text { SP-2 } & 53.23 & 29.36 & 0.805 & 13.14 & 0.232 & 3.94 & 0.011 & 100.72\end{array}$ $\begin{array}{llllllllll}200 \mathrm{R}-1,18-24 \mathrm{~cm} & \text { SP-2 } & 52.76 & 28.98 & 0.891 & 13.22 & 0.232 & 4.08 & 0.020 & 100.19\end{array}$ $\begin{array}{llllllllll}200 \mathrm{R}-1,18-24 \mathrm{~cm} & \text { SP-2 } & 52.05 & 29.15 & 0.902 & 13.46 & 0.211 & 3.72 & 0.020 & 99.51\end{array}$ $\begin{array}{llllllllll}200 \mathrm{R}-1,18-24 \mathrm{~cm} & \mathrm{SP}-2 & 52.80 & 29.59 & 0.920 & 13.23 & 0.215 & 3.48 & 0.023 & 100.26\end{array}$ $\begin{array}{lllllllllll}200 \mathrm{R}-1,18-24 \mathrm{~cm} & \mathrm{SP}-2 & 52.45 & 30.23 & 1.021 & 13.56 & 0.238 & 3.49 & 0.000 & 100.99\end{array}$

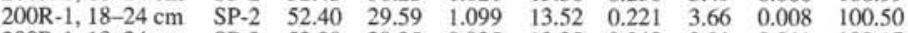
$\begin{array}{llllllllll}200 \mathrm{R}-1,18-24 \mathrm{~cm} & \mathrm{SP}-2 & 52.39 & 29.35 & 0.925 & 13.35 & 0.243 & 3.91 & 0.011 & 100.17\end{array}$ $\begin{array}{lllllllllr}200 \mathrm{R}-1,18-24 \mathrm{~cm} & \text { SP-2 } & 52.46 & 29.17 & 0.891 & 13.51 & 0.319 & 3.53 & 0.008 & 99.89 \\ 200 \mathrm{R}-1,18-24 \mathrm{~cm} & \text { SP-2 } & 55.31 & 29.88 & 1.077 & 13.63 & 0.300 & 3.65 & 0.004 & 100.84\end{array}$ \begin{tabular}{llllllllll}
$200 \mathrm{R}-1,18-24 \mathrm{~cm}$ & SP- & 52.31 & 29.88 & 1.077 & 13.63 & 0.300 & 3.65 & 0.004 & 100.84 \\
& SP.62 & 29.38 & 1.047 & 13.77 & 0.300 & 3.26 & 0.030 & 98.41 \\
\hline
\end{tabular} $\begin{array}{lllllllllll}200 \mathrm{R}-1,18-24 \mathrm{~cm} & \text { SP-3 } & 50.57 & 30.84 & 0.936 & 13.76 & 0.183 & 3.45 & 0.002 & 99.75\end{array}$ $\begin{array}{llllllllll}200 \mathrm{R}-1,18-24 \mathrm{~cm} & \text { SP-3 } & 50.43 & 30.26 & 0.849 & 13.92 & 0.209 & 3.28 & 0.008\end{array}$ $\begin{array}{lllllllll}200 \mathrm{R}-1,18-24 \mathrm{~cm} & \text { SP-3 } & 50.25 & 29.21 & 1.040 & 13.90 & 0.187 & 3.46 & 0.009\end{array}$ $\begin{array}{lllllllll}200 \mathrm{R}-1,18-24 \mathrm{~cm} & \text { SP-3 } & 50.11 & 29.19 & 0.906 & 13.98 & 0.210 & 3.12 & 0.007\end{array}$

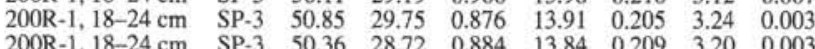
$\begin{array}{lllllllll}200 \mathrm{R}-1,18-24 \mathrm{~cm} & \text { SP-3 } & 50.36 & 28.72 & 0.884 & 13.84 & 0.209 & 3.20 & 0.003 \\ 200 \mathrm{R}-1,18-24 \mathrm{~cm} & \text { SP-3 } & 49.97 & 29.13 & 0.908 & 13.82 & 0.248 & 3.36 & 0.008\end{array}$ $\begin{array}{lllllllll}200 \mathrm{R}-1,18-24 \mathrm{~cm} & \text { SP-3 } & 49.97 & 29.13 & 0.908 & 13.82 & 0.248 & 3.36 & 0.008 \\ 200 \mathrm{R}-1,18-24 \mathrm{~cm} & \text { SP-3 } & 50.88 & 29.83 & 0.986 & 13.76 & 0.234 & 3.17 & 0.015\end{array}$ $\begin{array}{lllllllll}200 \mathrm{R}-1,18-24 \mathrm{~cm} & \text { SP-3 } & 51.16 & 28.85 & 0.875 & 13.69 & 0.282 & 3.21 & 0.007\end{array}$ $\begin{array}{lllllllll}200 \mathrm{R}-1,18-24 \mathrm{~cm} & \text { SP-3 } & 51.42 & 28.76 & 1.013 & 13.63 & 0.231 & 3.32 & 0.000\end{array}$ $\begin{array}{lllllllll}200 \mathrm{R}-1,18-24 \mathrm{~cm} & \text { SP-3 } & 51.42 & 28.76 & 1.013 & 13.63 & 0.231 & 3.32 & 0.000 \\ 200 \mathrm{R}-1,18-24 \mathrm{~cm} & \text { SP-3 } & 50.74 & 28.74 & 0.958 & 13.57 & 0.274 & 3.41 & 0.000\end{array}$ $\begin{array}{lllllllll}200 \mathrm{R}-1,18-24 \mathrm{~cm} & \text { SP-3 } & 50.74 & 28.74 & 0.958 & 13.57 & 0.274 & 3.41 & 0.000 \\ 200 \mathrm{R}-1,18-24 \mathrm{~cm} & \text { SP-4 } & 51.07 & 28.70 & 0.944 & 13.42 & 0.422 & 3.52 & 0.00\end{array}$ $\begin{array}{lllllllll}200 \mathrm{R}-1,18-24 \mathrm{~cm} & \text { SP-4 } & 50.93 & 28.58 & 0.835 & 13.29 & 0.193 & 3.37 & 0.002 \\ 200 \mathrm{R}-1,18-24 \mathrm{~cm} & \text { SP-4 } & 51.97 & 28.51 & 0.813 & 13.54 & 0.35 & 3.41 & 0.012\end{array}$ $\begin{array}{lllllllll}200 R-1,18-24 \mathrm{~cm} & \text { SP-4 } & 51.97 & 28.51 & 0.813 & 13.54 & 0.235 & 3.41 & 0.012 \\ 200 \mathrm{R}-1,18-24 \mathrm{~cm} & \text { SP-4 } & 53.13 & 27.77 & 0.902 & 12.13 & 0.219 & 3.73 & 0.018\end{array}$ $\begin{array}{lllllllll}200 \mathrm{R}-1,18-24 \mathrm{~cm} & \text { SP-4 } & 50.84 & 28.94 & 0.789 & 13.61 & 0.242 & 3.24 & 0.016\end{array}$ $\begin{array}{lllllllll}200 \mathrm{R}-1,18-24 \mathrm{~cm} & \text { SP-4 } & 51.25 & 29.39 & 0.812 & 13.60 & 0.220 & 3.42 & 0.007\end{array}$ $\begin{array}{lllllllll}200 \mathrm{R}-1,18-24 \mathrm{~cm} & \text { SP-4 } & 51.38 & 29.19 & 0.836 & 13.71 & 0.257 & 3.33 & 0.000\end{array}$ $\begin{array}{lllllllll}200 \mathrm{R}-1,18-24 \mathrm{~cm} & \text { SP- } 4 & 51.30 & 28.59 & 0.792 & 13.44 & 0.234 & 3.52 & 0.008\end{array}$ $\begin{array}{lllllllll}200 \mathrm{R}-1,18-24 \mathrm{~cm} & \text { SP-4 } & 51.08 & 28.18 & 0.792 & 13.25 & 0.261 & 3.62 & 0.007\end{array}$ $\begin{array}{lllllllll}200 \mathrm{R}-1,18-24 \mathrm{~cm} & \text { SP-4 } & 50.96 & 28.51 & 0.795 & 13.24 & 0.253 & 3.76 & 0.018 \\ 200 \mathrm{R}-1,18-24 \mathrm{~cm} & \text { SP-4 } & 51.46 & 28.52 & 0.908 & 13.11 & 0.275 & 3.70 & 0.003\end{array}$ $\begin{array}{lllllllll}200 \mathrm{R}-1,18-24 \mathrm{~cm} & \text { SP-4 } & 51.46 & 28.52 & 0.908 & 13.11 & 0.275 & 3.70 & 0.003 \\ 200 \mathrm{R}-1,18-24 \mathrm{~cm} & \text { SP-4 } & 51.44 & 28.70 & 0.741 & 13.22 & 0.270 & 3.77 & 0.009\end{array}$

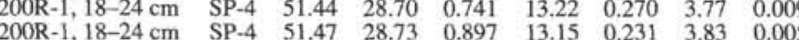

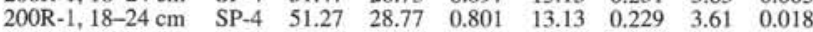
$\begin{array}{lllllllll}200 \mathrm{R}-1,18-24 \mathrm{~cm} & \text { SP-4 } & 51.54 & 28.47 & 0.738 & 12.92 & 0.242 & 3.81 & 0.000\end{array}$ $\begin{array}{lllllllll}200 \mathrm{R}-1,18-24 \mathrm{~cm} & \text { SP-4 } & 51.54 & 28.47 & 0.738 & 12.92 & 0.242 & 3.81 & 0.0010\end{array}$ $\begin{array}{lllllllll}200 R-1,18-24 \mathrm{~cm} & \text { SP-4 } & 51.92 & 29.00 & 0.842 & 13.01 & 0.246 & 3.81 & 0.010 \\ 200 \mathrm{R}-1,18-24 \mathrm{~cm} & \text { SP-5 } & 51.10 & 29.24 & 0.925 & 13.96 & 0.253 & 3.21 & 0.000\end{array}$

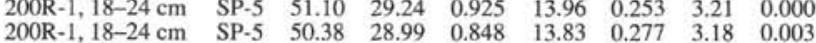
$\begin{array}{llllllllll}200 \mathrm{R}-1,18-24 \mathrm{~cm} & \text { SP-5 } & 50.45 & 28.81 & 0.794 & 13.61 & 0.305 & 3.48 & 0.000\end{array}$

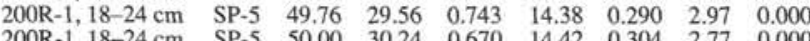
$\begin{array}{lllllllll}200 \mathrm{R}-1,18-24 \mathrm{~cm} & \text { SP-5 } & 50.00 & 30.24 & 0.670 & 14.42 & 0.304 & 2.77 & 0.000\end{array}$ $\begin{array}{lllllllll}200 \mathrm{R}-1,18-24 \mathrm{~cm} & \text { SP-5 } & 49.39 & 29.25 & 0.660 & 14.62 & 0.339 & 2.79 & 0.000\end{array}$

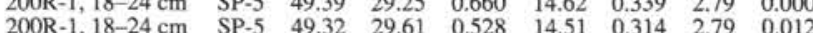

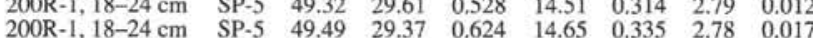
$\begin{array}{lllllllll}200 \mathrm{R}-1,18-24 \mathrm{~cm} & \text { SP-5 } & 49.99 & 30.22 & 0.642 & 14.69 & 0.344 & 2.73 & 0.010\end{array}$ $\begin{array}{lllllllll}200 \mathrm{R}-1,18-24 \mathrm{~cm} & \text { SP-5 } & 50.24 & 29.34 & 0.701 & 14.59 & 0.303 & 2.98 & 0.000\end{array}$ $\begin{array}{lllllllll}200 \mathrm{R}-1,18-24 \mathrm{~cm} & \text { SP-5 } & 50.31 & 29.50 & 0.801 & 14.17 & 0.282 & 3.09 & 0.006\end{array}$

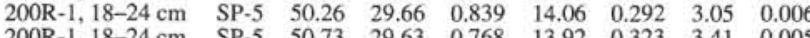
$\begin{array}{lllllllll}226 \mathrm{R}-1,8-12 \mathrm{~cm} & \text { SP-1 } & 50.31 & 29.02 & 0.742 & 14.11 & 0.178 & 3.24 & 0.017\end{array}$ $\begin{array}{lllllllll}226 \mathrm{R}-1,8-12 \mathrm{~cm} & \text { SP-1 } & 50.43 & 29.45 & 0.714 & 14.22 & 0.247 & 3.24 & 0.017\end{array}$ $\begin{array}{lllllllll}226 \mathrm{R}-1,8-12 \mathrm{~cm} & \text { SP-1 } & 50.43 & 29.45 & 0.714 & 14.22 & 0.247 & 3.16 & 0.001 \\ 226 \mathrm{R}-1,8-12 \mathrm{~cm} & \text { SP-1 } & 50.26 & 29.06 & 0.781 & 14.04 & 0.273 & 3.08 & 0.000\end{array}$ $\begin{array}{lllllllll}226 \mathrm{R}-1,8-12 \mathrm{~cm} & \text { SP-1 } & 50.26 & 29.06 & 0.781 & 14.04 & 0.273 & 3.08 & 0.000 \\ 226 \mathrm{R}-1,8-12 \mathrm{~cm} & \text { SP-2 } & 50.74 & 29.24 & 0.854 & 13.56 & 0.156 & 3.30 & 0.003\end{array}$ $\begin{array}{lllllllll}226 \mathrm{R}-1,8-12 \mathrm{~cm} & \text { SP-2 } & 51.03 & 28.94 & 0.828 & 13.76 & 0.183 & 3.44 & 0.012\end{array}$ $\begin{array}{lllllllll}226 \mathrm{R}-1,8-12 \mathrm{~cm} & \mathrm{SP}-2 & 51.00 & 28.82 & 0.867 & 13.74 & 0.164 & 3.61 & 0.020\end{array}$ $226 \mathrm{R}-1,8-12 \mathrm{~cm}$ $226 \mathrm{R}-1,8-12 \mathrm{~cm}$ $226 \mathrm{R}-1,8-12 \mathrm{~cm}$ $\begin{array}{llllllll}\text { SP-3 } & 52.36 & 28.88 & 0.997 & 13.23 & 0.179 & 3.53 & 0.000 \\ \text { SP-3 } & 51.79 & 29.55 & 0.837 & 13.82 & 0.215 & 3.57 & 0.019\end{array}$ $\begin{array}{lllllllll}\mathrm{SP}-3 & 52.35 & 27.99 & 0.836 & 13.11 & 0.208 & 3.66 & 0.012\end{array}$ 98.96 97.52 98.83 97.44 98.88 98.38 97.69 98.08 98.49
97.89 97.89
97.67 98.69 98.70 97.88
97.19 97.54
97.97 98.15 98.31
97.83 97.72

98.85 98.69
97.51 97.45
97.70 98.41 97.12 97.06 97.27 98.62 98.16 98.16 98.78 97.61 98.22
97.50 97.84 98.20
98.22

$\begin{array}{llllllllll}2.374 & 1.567 & 0.036 & 0.663 & 0.032 & 0.307 & 0.000 & 4.978 & 68.4 & 46.8\end{array}$ \begin{tabular}{lllllllllll}
2.381 & 1.565 & 0.033 & 0.670 & 0.017 & 0.308 & 0.000 & 4.974 & 68.5 & 33.8 \\
\hline
\end{tabular} $\begin{array}{llllllllll}2.400 & 1.523 & 0.034 & 0.682 & 0.028 & 0.309 & 0.001 & 4.977 & 68.7 & 45.7 \\ & 1.582 & 0.032 & 0.680 & 0.013 & 0.297 & 0.000 & 4.973 & 69.6 & 29.3\end{array}$ $\begin{array}{llllllllll}2.390 & 1.558 & 0.029 & 0.662 & 0.014 & 0.327 & 0.001 & 4.981 & 66.9 & 32.7\end{array}$ $\begin{array}{llllllllll}2.382 & 1.567 & 0.032 & 0.659 & 0.016 & 0.321 & 0.001 & 4.979 & 67.2 & 33.9\end{array}$ $\begin{array}{llllllllll}2.382 & 1.567 & 0.032 & 0.659 & 0.016 & 0.321 & 0.001 & 4.979 & 67.2 & 33.9 \\ 2.373 & 1.575 & 0.033 & 0.659 & 0.023 & 0.315 & 0.001 & 4.981 & 67.6 & 40.9\end{array}$ $\begin{array}{llllllllll}2.381 & 1.567 & 0.030 & 0.663 & 0.019 & 0.322 & 0.000 & 4.982 & 67.3 & 38.0\end{array}$ $\begin{array}{lllllllllll}2.381 & 1.567 & 0.030 & 0.663 & 0.019 & 0.322 & 0.000 & 4.982 & 67.3 & 38.0 \\ 2.389 & 1.550 & 0.034 & 0.675 & 0.014 & 0.312 & 0.001 & 4.975 & 68.3 & 28.7\end{array}$ $\begin{array}{llllllllll}2.387 & 1.559 & 0.034 & 0.659 & 0.019 & 0.317 & 0.001 & 4.975 & 67.5 & 36.0\end{array}$ $\begin{array}{llllllllll}2.363 & 1.596 & 0.038 & 0.652 & 0.014 & 0.312 & 0.001 & 4.977 & 67.6 & 26.5\end{array}$ $\begin{array}{llllllllll}2.367 & 1.591 & 0.033 & 0.662 & 0.013 & 0.311 & 0.000 & 4.976 & 68.1 & 28.0\end{array}$ $\begin{array}{llllllllll}2.391 & 1.549 & 0.032 & 0.661 & 0.015 & 0.337 & 0.001 & 4.986 & 66.2 & 31.4\end{array}$ $\begin{array}{llllllllll}2.396 & 1.554 & 0.031 & 0.658 & 0.014 & 0.318 & 0.000 & 4.971 & 67.4 & 31.9\end{array}$ $\begin{array}{llllllllll}2.369 & 1.593 & 0.028 & 0.666 & 0.014 & 0.298 & 0.001 & 4.969 & 69.1 & 33.3\end{array}$ $\begin{array}{llllllllll}2.407 & 1.548 & 0.029 & 0.651 & 0.016 & 0.304 & 0.000 & 4.956 & 68.2 & 36.3\end{array}$ $\begin{array}{llllllllll}2.460 & 1.488 & 0.031 & 0.603 & 0.011 & 0.373 & 0.001 & 4.967 & 61.8 & 25.7\end{array}$ $\begin{array}{llllllllll}2.399 & 1.545 & 0.029 & 0.667 & 0.025 & 0.296 & 0.001 & 4.962 & 69.2 & 46.6 \\ \end{array}$ $\begin{array}{llllllllll}2.406 & 1.533 & 0.033 & 0.667 & 0.017 & 0.308 & 0.001 & 4.965 & 68.4 & 34.2\end{array}$ $\begin{array}{llllllllll}2.413 & 1.537 & 0.031 & 0.651 & 0.015 & 0.310 & 0.001 & 4.959 & 67.6 & 33.2 \\ & 1.536 & 0.028 & 0.648 & 0.015 & 0.325 & 0.001 & 4.967 & 66.6 & 35.4\end{array}$ \begin{tabular}{llllllllll}
2.414 & 1.536 & 0.028 & 0.648 & 0.015 & 0.325 & 0.001 & 4.967 & 66.6 & 35.4 \\
\hline & 1.531 & 0.031 & 0.645 & 0.016 & 0.334 & 0.001 & 4.972 & 65.8 & 34.4
\end{tabular} $\begin{array}{lllllllllll}2.414 & 1.531 & 0.031 & 0.645 & 0.016 & 0.334 & 0.001 & 4.972 & 65.8 & 34.4 \\ 2.421 & 1.532 & 0.027 & 0.637 & 0.013 & 0.340 & 0.002 & 4.971 & 65.1 & 33.0\end{array}$ $\begin{array}{lllllllllll}2.421 & 1.532 & 0.027 & 0.637 & 0.013 & 0.340 & 0.002 & 4.971 & 65.1 & 33.0 \\ 2.425 & 1.533 & 0.034 & 0.599 & 0.013 & 0.374 & 0.001 & 4.979 & 61.5 & 27.7\end{array}$ $\begin{array}{llllllllll}2.425 & 1.533 & 0.034 & 0.599 & 0.013 & 0.374 & 0.001 & 4.979 & 61.5 & 27.7 \\ 2.413 & 1.545 & 0.026 & 0.615 & 0.013 & 0.374 & 0.000 & 4.988 & 62.1 & 32.9\end{array}$ $\begin{array}{llllllllll}2.419 & 1.543 & 0.033 & 0.614 & 0.012 & 0.339 & 0.001 & 4.963 & 64.3 & 27.1\end{array}$ \begin{tabular}{llllllllll}
2.399 & 1.559 & 0.027 & 0.634 & 0.016 & 0.344 & 0.001 & 4.980 & 64.8 & 36.4 \\
\hline
\end{tabular} $\begin{array}{llllllllll}2.395 & 1.550 & 0.030 & 0.643 & 0.016 & 0.359 & 0.001 & 4.995 & 64.1 & 34.0\end{array}$ $\begin{array}{llllllllll}2.379 & 1.570 & 0.031 & 0.659 & 0.014 & 0.330 & 0.001 & 4.985 & 66.6 & 31.7 \\ & 1.578 & 0.031 & 0.641 & 0.015 & 0.305 & 0.001 & 4.960 & 67.7 & 31.6\end{array}$ \begin{tabular}{llllllllll}
2.389 & 1.578 & 0.031 & 0.641 & 0.015 & 0.305 & 0.001 & 4.960 & 67.7 & 31.6 \\
\hline & 1.603 & 0.035 & 0.654 & 0.016 & 0.304 & 0.000 & 4.973 & 68.2 & 31.6
\end{tabular} $\begin{array}{llllllllll}2.361 & 1.603 & 0.035 & 0.654 & 0.016 & 0.304 & 0.000 & 4.973 & 68.2 & 31.6 \\ 2.372 & 1.579 & 0.037 & 0.656 & 0.015 & 0.321 & 0.000 & 4.981 & 67.1 & 28.5\end{array}$ $\begin{array}{llllllllll}2.379 & 1.571 & 0.032 & 0.649 & 0.016 & 0.344 & 0.001 & 4.992 & 65.3 & 34.2\end{array}$ $\begin{array}{llllllllll}2.386 & 1.564 & 0.031 & 0.658 & 0.022 & 0.311 & 0.000 & 4.972 & 67.9 & 41.5\end{array}$ $\begin{array}{llllllllll}2.361 & 1.589 & 0.037 & 0.659 & 0.020 & 0.319 & 0.000 & 4.986 & 67.4 & 35.6 \\ \end{array}$ $\begin{array}{llllllllll}2.344 & 1.604 & 0.036 & 0.683 & 0.021 & 0.293 & 0.002 & 4.983 & 69.9 & 36.2 \\ \end{array}$ \begin{tabular}{lllllllllll}
2.310 & 1.661 & 0.032 & 0.674 & 0.012 & 0.306 & 0.000 & 4.996 & 68.8 & 27.9 \\
\hline & 1.643 & 0.029 & 0.687 & 0.014 & 0.293 & 0.000 & 4.989 & 70.1 & 32.8
\end{tabular} $\begin{array}{llllllllll}2.322 & 1.643 & 0.029 & 0.687 & 0.014 & 0.293 & 0.000 & 4.989 & 70.1 & 32.8 \\ 2.339 & 1.603 & 0.036 & 0.693 & 0.013 & 0.312 & 0.001 & 4.998 & 68.9 & 26.3\end{array}$ $\begin{array}{llllllllll}2.339 & 1.603 & 0.036 & 0.693 & 0.013 & 0.312 & 0.001 & 4.998 & 68.9 & 26.3 \\ 2.342 & 1.608 & 0.032 & 0.700 & 0.015 & 0.283 & 0.000 & 4.980 & 71.2 & 31.5\end{array}$ $\begin{array}{llllllllll}2.343 & 1.616 & 0.030 & 0.687 & 0.014 & 0.289 & 0.000 & 4.979 & 70.3 & 31.7\end{array}$ $\begin{array}{llllllllll}2.359 & 1.586 & 0.031 & 0.695 & 0.015 & 0.291 & 0.000 & 4.977 & 70.5 & 31.9\end{array}$ $\begin{array}{llllllllll}2.339 & 1.607 & 0.032 & 0.693 & 0.017 & 0.305 & 0.000 & 4.994 & 69.4 & 35.1\end{array}$ $\begin{array}{llllllllll}2.342 & 1.619 & 0.034 & 0.679 & 0.016 & 0.283 & 0.001 & 4.973 & 70.5 & 32.0\end{array}$ $\begin{array}{llllllllll}2.372 & 1.577 & 0.031 & 0.680 & 0.019 & 0.289 & 0.000 & 4.968 & 70.2 & 39.0\end{array}$ $\begin{array}{llllllllll}2.378 & 1.568 & 0.035 & 0.675 & 0.016 & 0.298 & 0.000 & 4.970 & 69.4 & 31.1\end{array}$ $\begin{array}{llllllllll}2.365 & 1.579 & 0.034 & 0.678 & 0.019 & 0.308 & 0.000 & 4.983 & 68.7 & 36.2\end{array}$ $\begin{array}{llllllllll}2.370 & 1.570 & 0.033 & 0.667 & 0.029 & 0.317 & 0.000 & 4.987 & 67.8 & 47.0\end{array}$ $\begin{array}{llllllllll}2.381 & 1.575 & 0.029 & 0.666 & 0.013 & 0.305 & 0.000 & 4.970 & 68.5 & 31.4\end{array}$ $\begin{array}{llllllllll}2.397 & 1.550 & 0.028 & 0.669 & 0.016 & 0.305 & 0.001 & 4.967 & 68.6 & 36.4 \\ \end{array}$ $\begin{array}{llllllllll}2.452 & 1.511 & 0.031 & 0.600 & 0.015 & 0.334 & 0.001 & 4.944 & 64.2 & 32.5 \\ & 1.588 & 0.028 & 0.679 & 0.017 & 0.292 & 0.001 & 4.972 & 69.8 & 37.8\end{array}$ $\begin{array}{llllllllll}2.362 & 1.597 & 0.028 & 0.672 & 0.015 & 0.306 & 0.000 & 4.979 & 68.7 & 34.9\end{array}$ $\begin{array}{llllllllll}2.367 & 1.586 & 0.029 & 0.677 & 0.018 & 0.298 & 0.000 & 4.974 & 69.5 & 37.8\end{array}$ $\begin{array}{llllllllll}2.383 & 1.566 & 0.028 & 0.669 & 0.016 & 0.317 & 0.000 & 4.979 & 67.8 & 36.9\end{array}$ \begin{tabular}{llllllllll}
2.383 & 1.566 & 0.028 & 0.669 & 0.016 & 0.317 & 0.000 & 4.979 & 67.8 & 36.9 \\
\hline .390 & 1.554 & 0.028 & 0.664 & 0.018 & 0.328 & 0.000 & 4.983 & 66.9 & 39.5
\end{tabular} $\begin{array}{llllllllll}2.378 & 1.568 & 0.028 & 0.662 & 0.018 & 0.340 & 0.001 & 4.995 & 66.0 & 38.7\end{array}$ $\begin{array}{llllllllll}2.388 & 1.560 & 0.032 & 0.652 & 0.019 & 0.333 & 0.000 & 4.983 & 66.2 & 37.5\end{array}$ $\begin{array}{llllllllll}2.383 & 1.567 & 0.026 & 0.656 & 0.019 & 0.339 & 0.001 & 4.990 & 65.9 & 41.9\end{array}$ $\begin{array}{llllllllll}2.381 & 1.567 & 0.031 & 0.652 & 0.016 & 0.344 & 0.000 & 4.991 & 65.5 & 33.8 \\ & 1.575 & 0.028 & 0.653 & 0.016 & 0.325 & 0.001 & 4.980 & 66.7 & 36.2\end{array}$ $\begin{array}{llllllllll}2.395 & 1.560 & 0.026 & 0.643 & 0.017 & 0.343 & 0.000 & 4.984 & 65.2 & 39.4\end{array}$ $\begin{array}{llllllllll}2.395 & 1.560 & 0.026 & 0.643 & 0.017 & 0.343 & 0.000 & 4.984 & 65.2 & 39.4 \\ 2.386 & 1.571 & 0.029 & 0.641 & 0.017 & 0.339 & 0.001 & 4.984 & 65.3 & 36.7\end{array}$ \begin{tabular}{lllllllllll}
2.386 & 1.571 & 0.029 & 0.641 & 0.017 & 0.339 & 0.001 & 4.984 & 65.3 & 36.7 \\
\hline .358 & 1.590 & 0.032 & 0.690 & 0.017 & 0.287 & 0.000 & 4.975 & 70.6 & 35.1
\end{tabular} $\begin{array}{llllllllll}2.353 & 1.596 & 0.030 & 0.692 & 0.019 & 0.288 & 0.000 & 4.978 & 70.6 & 39.3\end{array}$ $\begin{array}{llllllllll}2.358 & 1.588 & 0.028 & 0.682 & 0.021 & 0.315 & 0.000 & 4.992 & 68.4 & 43.2\end{array}$ $\begin{array}{llllllllll}2.323 & 1.627 & 0.026 & 0.719 & 0.020 & 0.269 & 0.000 & 4.985 & 72.8 & 43.6 \\ & 1.650 & 0.023 & 0.715 & 0.021 & 0.249 & 0.000 & 4.973 & 74.2 & 47.3\end{array}$ $\begin{array}{llllllllll}2.315 & 1.650 & 0.023 & 0.715 & 0.021 & 0.249 & 0.000 & 4.973 & 74.2 & 47.3 \\ \end{array}$ $\begin{array}{llllllllll}.323 & 1.622 & 0.023 & 0.737 & 0.024 & 0.254 & 0.000 & 4.982 & 74.3 & 50.4\end{array}$ \begin{tabular}{lllllllllll}
2.323 & 1.622 & 0.023 & 0.737 & 0.024 & 0.254 & 0.000 & 4.982 & 74.3 & 50.4 \\
\hline & 1.640 & 0.019 & 0.730 & 0.022 & 0.254 & 0.001 & 4.982 & 74.1 & 54.1
\end{tabular} $\begin{array}{llllllllll}2.316 & 1.640 & 0.019 & 0.730 & 0.022 & 0.254 & 0.001 & 4.982 & 74.1 & 54.1 \\ 2.322 & 1.624 & 0.022 & 0.736 & 0.023 & 0.253 & 0.001 & 4.982 & 74.4 & 51.5\end{array}$ $\begin{array}{llllllllll}2.311 & 1.647 & 0.022 & 0.728 & 0.024 & 0.245 & 0.001 & 4.977 & 74.8 & 51.5\end{array}$ $\begin{array}{llllllllll}2.335 & 1.608 & 0.025 & 0.727 & 0.021 & 0.269 & 0.000 & 4.983 & 73.0 & 46.1\end{array}$ $\begin{array}{llllllllll}2.336 & 1.615 & 0.028 & 0.705 & 0.020 & 0.278 & 0.000 & 4.982 & 71.7 & 41.1 \\ & 1.623 & 0.029 & 0.699 & 0.020 & 0.274 & 0.000 & 4.979 & 71.8 & 40.8\end{array}$ $\begin{array}{llllllllll}2.333 & 1.623 & 0.029 & 0.699 & 0.020 & 0.274 & 0.000 & 4.979 & 71.8 & 40.8 \\ \end{array}$ $\begin{array}{llllllllll}2.349 & 1.598 & 0.026 & 0.706 & 0.012 & 0.293 & 0.001 & 4.986 & 70.6 & 32.2\end{array}$ $\begin{array}{lllllllllll}2.340 & 1.611 & 0.025 & 0.707 & 0.017 & 0.284 & 0.000 & 4.984 & 71.3 & 40.7\end{array}$ $\begin{array}{llllllllll}2.340 & 1.611 & 0.025 & 0.707 & 0.017 & 0.284 & 0.000 & 4.984 & 71.3 & 40.7 \\ 2.348 & 1.600 & 0.027 & 0.703 & 0.019 & 0.279 & 0.000 & 4.977 & 71.6 & 40.9\end{array}$ $\begin{array}{lllllllllll}2.359 & 1.602 & 0.030 & 0.675 & 0.011 & 0.297 & 0.000 & 4.974 & 69.4 & 26.6\end{array}$ $\begin{array}{llllllllll}2.366 & 1.582 & 0.029 & 0.684 & 0.013 & 0.310 & 0.001 & 4.984 & 68.8 & 30.4\end{array}$ $\begin{array}{lllllllllll}2.367 & 1.576 & 0.030 & 0.683 & 0.011 & 0.325 & 0.001 & 4.993 & 67.7 & 27.3 \\ \end{array}$ $\begin{array}{lllllllllll}2.363 & 1.589 & 0.029 & 0.676 & 0.015 & 0.315 & 0.001 & 4.987 & 68.1 & 33.7\end{array}$ $\begin{array}{llllllllll}2.399 & 1.561 & 0.028 & 0.655 & 0.015 & 0.298 & 0.001 & 4.956 & 68.7 & 35.0\end{array}$ 
Table 6 (continued).

\begin{tabular}{|c|c|c|c|c|c|c|c|c|c|c|c|c|c|c|c|c|c|c|c|}
\hline \multirow[b]{2}{*}{ Sample } & \multirow[b]{2}{*}{ Grain } & \multicolumn{8}{|c|}{ Weight percent } & \multicolumn{10}{|c|}{ Cations per 8 oxygens } \\
\hline & & $\mathrm{iO}_{2}$ & $\mathrm{l}_{2} \mathrm{O}_{3}$ & $\mathrm{e}_{2} \mathrm{O}_{3}$ & $\mathrm{CaO}$ & $\mathrm{MgO}$ & $\mathrm{Na}_{2} \mathrm{O}$ & $\mathrm{K}_{2} \mathrm{O}$ & Total & $\mathrm{Si}$ & $\mathrm{Al}$ & $\mathrm{Fe}$ & $\mathrm{Ca}$ & $\mathrm{Mg}$ & $\mathrm{Na}$ & K & Total & An\% & $\mathrm{Mg} \#$ \\
\hline \multicolumn{20}{|c|}{ Radial plagioclase splay tips } \\
\hline $200 \mathrm{R}-1,18-24 \mathrm{~cm}$ & SP-1t & 54.84 & 0 & 315 & .58 & 3 & 4.67 & 013 & 00.24 & 2.475 & 473 & 045 & 0.560 & .008 & 0.409 & 0.001 & 4.971 & 57.8 & 15.6 \\
\hline $24 \mathrm{~cm}$ & SP-1t & & & 050 & 12.06 & 137 & 4.40 & .020 & 100.45 & 2.450 & 1.508 & .036 & 0.582 & .009 & 0.385 & 0.001 & & & \\
\hline$-24 \mathrm{~cm}$ & SP-1t & 54.23 & 8.24 & 896 & 12.18 & 163 & 4.44 & .025 & 100.17 & .451 & 1.504 & .030 & 0.590 & 0.011 & 0.389 & 0.001 & & .2 & 5.5 \\
\hline $200 \mathrm{R}-1,18-24 \mathrm{~cm}$ & SP-1t & 53.53 & 27.91 & 1.055 & 12.09 & 0.168 & 4.38 & .009 & 99.14 & 446 & 1.503 & .036 & 0.592 & 0.011 & 0.388 & 0.001 & 4.978 & 60.4 & 24.0 \\
\hline$-24 \mathrm{~cm}$ & SP-1t & 53.71 & 8.33 & .099 & 12.16 & .186 & 4.41 & .015 & & & 1.515 & .038 & 0.591 & 0.013 & 0.388 & 0.001 & & 3 & 5.1 \\
\hline $4 \mathrm{~cm}$ & SP-1t & 54.61 & 8.44 & .102 & 12.02 & .193 & 4.50 & 0.023 & 100 & 1 & & 37 & 0.578 & 13 & 0.391 & 0.001 & 4.975 & 59.5 & 5.8 \\
\hline $200 \mathrm{R}-1,1$ & SP-1t & 53.94 & 27.91 & 1.056 & 11.77 & 0.180 & 4.73 & 0.020 & 99.59 & 453 & 1.496 & 36 & 0.573 & 12 & 0.417 & 0.001 & 4.990 & 57.8 & 25.2 \\
\hline$-24 \mathrm{~cm}$ & SP-1t & 54.36 & 27.45 & 1.260 & 11.60 & 0.141 & 4.59 & 0.000 & 99.40 & & 1.472 & & & & & 0.000 & & 58.3 & 18.1 \\
\hline $\mathrm{cm}$ & SP-1t & 9 & 770 & & 1020 & 3 & 5.40 & .012 & & & & & & & & & & & 8.0 \\
\hline $4 \mathrm{~cm}$ & SP-1t & 56.14 & & & & 5 & 5.0 & & & 1 & & & 0.509 & & & 0.002 & 4.966 & 53.6 & 13.7 \\
\hline$-24 \mathrm{~cm}$ & SP-1t & 56.14 & 27.44 & 1.412 & 10.92 & 0.1 & 5.38 & 0.038 & & 2.502 & 1.441 & & 0.521 & & & 0.002 & & 52.7 & 15.1 \\
\hline $\mathrm{cm}$ & SP-1t & & 2740 & & 11.12 & 5 & 4.87 & 0.026 & & 06 & & & & & & & & & 19.1 \\
\hline $\mathrm{cm}$ & SP-1t & & & & & 5 & 6.18 & & & 2606 & & & & & & 0.001 & & 44.6 & 9.7 \\
\hline $000 \mathrm{R}-1$ & SP-1t & 59.55 & 25.12 & 0.892 & 7.91 & 0.025 & 6.7 & 0.0 & & 53 & 1.319 & 0. & 0. & 0.0 & & 0.003 & & 39.2 & 5.3 \\
\hline $4 \mathrm{~cm}$ & SP-2t & & & & 8 & 3 & 4. & 3 & & & & & & & & & & 57.8 & 15.6 \\
\hline $\mathrm{cm}$ & SP-2t & & & & & 7 & 4.4 & 0 & & & & & 0.5 & & & 0.001 & 4.971 & 60.1 & 20.5 \\
\hline $24 \mathrm{~cm}$ & SP-2t & 54.23 & 28.24 & 0.896 & 12.18 & 3 & 4.44 & 0.025 & 100 . & 1 & 1.504 & 0. & 0.590 & 0. & 0.3 & 0.001 & 4.977 & 60.2 & 26.5 \\
\hline$-24 \mathrm{~cm}$ & SP- $2 t$ & 53.53 & 27.91 & 1.055 & 12.09 & 0.1 & 4.38 & 0.009 & 99 & 16 & 1.503 & 36 & 0.592 & & & 0.001 & & 60.4 & 4.0 \\
\hline $\mathrm{cm}$ & SP- & & & & & & 1 & & & & & & & & & & & 3 & 1 \\
\hline $4 \mathrm{~cm}$ & $S P-2 t$ & 5 & & & & & 4.50 & & 100 & & & & & & & 0.001 & & 59.5 & 25.8 \\
\hline$-24 \mathrm{~cm}$ & SP-2t & 53.94 & 27.91 & 1.056 & 11.77 & 0.180 & 4.73 & 0.020 & 99.59 & 2.453 & 1.496 & 0.036 & 0.5 & & 0.417 & 0.001 & 4.990 & 57.8 & 25.2 \\
\hline $4 \mathrm{~cm}$ & SP-2t & & 7 & & 1160 & 01 & 4.59 & 0.000 & & & & & & & & & & & 18.1 \\
\hline $\mathrm{cm}$ & SP-2t & & 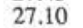 & & & & 5.4 & & & & & & & & & 0.0 & & 51.0 & 8.0 \\
\hline$-24 \mathrm{~cm}$ & SP-2t & 56.14 & 27.52 & & 10.67 & 0.1 & 5.08 & 0.035 & & 2.5 & 1.445 & & 0.5 & & & 0.002 & 4.966 & 53.6 & 13.7 \\
\hline $24 \mathrm{~cm}$ & SP-2t & & & & 10. & 01 & 5.38 & 0.038 & 46 & 2.502 & 1.441 & & & & & & & 52.7 & 15.1 \\
\hline $\mathrm{cm}$ & SP- $2 t$ & & & & 11.12 & . & 487 & 0006 & & & & & & & & & & 55.7 & 19.1 \\
\hline$-24 \mathrm{~cm}$ & SP-2t & 58.43 & & & & & 6.1 & & & & & & & & & 0.6 & & 44.6 & 9.7 \\
\hline $200 \mathrm{R}-1,18-24 \mathrm{~cm}$ & SP-2t & 59.55 & 25.12 & 0.892 & 7.91 & 0.025 & 6.74 & 0.047 & 100.29 & 2.653 & 1.319 & 0.030 & 0.378 & 0. & 0.5 & 0.003 & & 39.2 & 5.3 \\
\hline $1,8-12 \mathrm{~cm}$ & SP-3t & 53.49 & 28.31 & 1.169 & 12.31 & 0.126 & 4.20 & 0.026 & & 2.434 & & & 0.600 & & & 0.002 & 4.973 & 61.7 & 17.6 \\
\hline $\mathrm{cm}$ & SP-3 & & & & & & 4.0 & & & & & & & & & & & 63.6 & 22.6 \\
\hline $226 \mathrm{R}-1,8-12 \mathrm{~cm}$ & SP-3t & 52.25 & 27.82 & 1.0 & 12. & 0.1 & 3.9 & 0.0 & & & 1.5 & 0.0 & 0.6 & & & 0.0 & & 64.4 & 21.3 \\
\hline $226 \mathrm{R}-1,8-12 \mathrm{~cm}$ & SP-3t & 52.88 & 28.16 & 0.960 & 13.12 & 0.178 & 3.96 & 0.014 & 99. & 2.420 & 1.519 & 0.0 & 0.6 & & & & & 64.6 & 26.9 \\
\hline & SP-3t & 54.97 & & & & & 4.98 & 0.022 & & & & & & & & & & 55.1 & 145 \\
\hline 226 & SP-3 & 53. & 27. & & & & 4. & & & & & & & & & & & 63.1 & 25.8 \\
\hline $226 \mathrm{R}-1,8-12 \mathrm{~cm}$ & SP-3t & 53.05 & 28.07 & 0.999 & 13.01 & 0.201 & 3.72 & 0.015 & 99.06 & 2.429 & 1.515 & 0.034 & 0.638 & 0.014 & 0.330 & 0.001 & 4.962 & 65.8 & 28.5 \\
\hline
\end{tabular}

line the tips of the plagioclase crystals, suggesting that when the crystallization of the individual plagioclase crystals ceased, the driving force for local pyroxene crystallization also ended.

In general, the length of plagioclase grains decreased from the phenocryst stage to the microphenocryst stage, after which it increased and then remained relatively constant from the elongate stage to the skeletal stage, and to the radial growth stage. As crystallization proceeded, the volume of individual grains decreased and the aspect ratio increased. Pyroxene crystal size also initially decreased from the phenocryst stage to the microphenocryst stage and then increased from the microphenocryst stage to the ophitic stage and from the ophitic stage to the poikilitic stage. The volume of individual pyroxene grains, in general, increased as crystallization proceeded, and the aspect ratio remained relatively constant. The plagioclase nucleation rate appears to have increased as crystallization proceeded while the pyroxene nucleation rate decreased. Gray (1970) reports similar changes in the relative nucleation rates of plagioclase and pyroxene from the margins of thick dikes towards their centers (pyroxene nucleation rates decrease more rapidly than plagioclase nucleation rates; plagioclase nucleation/pyroxene nucleation increases), resulting in ophitic and poikilitic textures in the cores of larger dikes. The textures and grainsizes in the diabase samples recovered on Legs 137 and 140 are compatible with crystallization in the cores of thick dikes or thin sills.

Experimental studies suggest that crystals with acicular, curved, skeletal, or branching morphologies grow under conditions where the growth rate is high relative to the diffusion rate (Kirkpatrick, 1975). These conditions can occur in settings where melt becomes supersaturated as a result of a significant degree of undercooling. The change from equant crystals to elongate, skeletal, curved, and finally branching crystals is indicative of a system with an increasing degree of undercooling as crystallization proceeded (Dowty, 1980; Lofgren, 1980). Experimental studies of plagioclase crystallization from plagioclase- $\mathrm{H}_{2} \mathrm{O}$ melts suggest cooling rates on the order of $>10^{\circ} \mathrm{C}$ per hr and undercooling in excess of $100^{\circ} \mathrm{C}$ to produce the degree of supersaturation necessary to form elongate crystals, and rates $>50^{\circ} \mathrm{C}$ per hr and undercooling in excess of $200^{\circ} \mathrm{C}$ to produce the degree of supersaturation necessary to form branching crystals (Lofgren, 1974; Lofgren and Donaldson, 1975). Experimental studies of lunar basalts indicate that cooling rates of 2 to $10^{\circ} \mathrm{C}$ per hr will produce elongate to skeletal pyroxene crystals, and that cooling rates $>10^{\circ} \mathrm{C}$ per hr will produce dendritic to spherulitic pyroxene crystals (Lofgren, 1980). Movement within a melt increases the nucleation rate so that larger degrees of undercooling are required to produce supersaturation textures in a moving magma than in a stagnant magma (Kouchi et al., 1986). In experimental studies of oceanic tholeiites, subophitic textures similar to those observed in the 504B diabase, but without the splays of radial plagioclase crystals, were produced at cooling rates of $2^{\circ} \mathrm{C}$ per hr in samples containing preexisting plagioclase nuclei (Lofgren 1980, 1983). In the 504B diabase, the degree of supersaturation in plagioclase appears to have increased with crystallization, suggesting that the melts did not contain preexisting plagioclase nuclei. The 504B diabase textures, therefore, indicate that individual dikes in the Hole 504B sequence at depths up to $1.7 \mathrm{~km}$ below seafloor, at time of emplacement, probably cooled at rates in excess of $2^{\circ} \mathrm{per} \mathrm{hr}$, and may have solidified in a matter of a few hours or days.

Very little correlation is observed between crystal morphology and crystal composition for pyroxene in the medium-grained diabase of Hole 504B. Phenocrysts, microphenocrysts, ophitic pyroxene, and poikilitic pyroxene all follow the same general zoning patterns with core compositions appropriate for crystallization in situ from tholeiitic melt. The fact that the crystals are strongly zoned and preserve fine scale zoning patterns, clearly demonstrates that original compositional differences have not been removed by post-crystallization re-equilibration. There is no evidence that the large $\mathrm{Cr}$-rich phenocrysts were formed elsewhere and subsequently incorporated in the magma that fed these dikes. Pyroxene and olivine crystals in the 504B diabase do not have the elongate, skeletal, or branching textures (cellular morphologies) characteristic of growth under supersaturation conditions. Experimental studies, however, indicate that pyroxene and olivine develop cellular morphologies at similar or lower 
degrees of supersaturation than that necessary to produce cellular morphologies in plagioclase. The magmas from which the 504B diabase crystallized must have been emplaced with preexisting olivine and pyroxene nuclei, but with few preexisting plagioclase nuclei. As a result, pyroxene and olivine crystallization and morphology were controlled by their growth rates while plagioclase crystallization and morphology was controlled by its nucleation rate. Variations in nucleation rates between minerals can effect the order in which they appear upon cooling (Gibb, 1974; Tsuchiyama, 1983), and therefore, the order in which they are fractionated and the relative amounts removed by fractional crystallization. The suppression of plagioclase nucleation and crystallization relative to that of pyroxene and olivine could provide a mechanism by which the actual fractionation assemblage is more pyroxene-rich and plagioclase-poor than that predicted from thermodynamic models (see Naslund et al., this volume), or that observed in isothermal crystallization experiments.

\section{ACKNOWLEDGMENTS}

This study has been supported in part by post-cruise funding from USSAC. William Blackburn assisted with the microprobe analyses, Anne Hull assisted with the drafting, and David Tuttle assisted with the photography. The manuscript was improved by helpful comments from Drs. C.I. Chalokwu, G.E. Lofgren, and H.B. Dick.

\section{REFERENCES $*$}

Bloomer, S.H., Natland, J.H., and Fisher, R.L., 1989. Mineral relationships in gabbroic rocks from fracture zones of Indian Ocean ridges: evidence for extensive fractionation, parental diversity, and boundary-layer recrystallization. In Saunders, A.D., and Norry, M.J. (Eds.), Magmatism in the Oceanic Basins. Geol. Soc. Spec. Publ. London, 42:107-124.

Bollinger, C., and Semet, M., 1980. Chemical zonation of plagioclase phenocrysts from Leg 51, 52, and 53 basalts. In Donnelly, T., Francheteau, J., Bryan, W., Robinson, P., Flower, M., Salisbury, M., et al., Init. Repts. DSDP, 51, 52, 53 (Pt. 2): Washington (U.S. Govt. Printing Office), 1055-1061.

Dowty, E., 1980. Crystal growth and nucleation theory and the numerical simulation of igneous crystallization. In Hargraves, R.B. (Ed.), Physics of Magmatic Processes: Princeton (Princeton Univ. Press), 419-486.

Gibb, F.G.F., 1974. Supercooling and crystallization of plagioclase from a basaltic magma. Mineral. Mag., 39:641-653.

Gray, N.H., 1970. Crystal growth and nucleation in two large diabase dikes. Can. J. Earth Sci., 7:366-375.
Grove, T.L., and Bryan, W.B., 1983. Fractionation of pyroxene-phyric MORB at low pressure: an experimental study. Contrib. Mineral. Petrol., 84:293-309.

Kirkpatrick, R.J., 1975. Crystal growth from the melt: a review. Am. Mineral., 60:798-814.

Koloskov, A.V., and Zharinov, S.E., 1993. Multivariate statistical analysis of clinopyroxene compositions from mafic and ultramafic xenoliths in volcanic rocks. J. Petrol., 34:173-185.

Kouchi, A., Tsuchiyama, A., and Sunagawa, I., 1986. Effect of stirring on crystallization kinetics of basalt: texture and element partitioning. Contrib. Mineral. Petrol., 93:429-438.

Lofgren, G., 1974. An experimental study of plagioclase crystal morphology: isothermal crystallization. Am. J. Sci., 274:243-273.

+1980 . Experimental studies on the dynamic crystallization of silicate melts. In Hargraves, R.B. (Ed.), Physics of Magmatic Processes: Princeton (Princeton Univ. Press), 487-565.

Lofgren, G.E., 1983. Effect of heterogeneous nucleation on basaltic textures: a dynamic crystallization study. J. Petrol., 24:229-255.

Lofgren, G.E., and Donaldson, C.H., 1975. Curved branching crystals and differentiation in comb-layered rocks. Contrib. Mineral. Petrol., 49:309-319.

Loucks, R.R., 1990. Discrimination of ophiolitic from nonophiolitic ultramafic-mafic allochthons in orogenic belts by the Al/Ti ratio in clinopyroxene. Geology, 18:346-349.

Sato, H., 1989. Mg-Fe partitioning between plagioclase and liquid in basalts of Hole 504B, ODP Leg 111: a study of melting at $1 \mathrm{~atm}$. In Becker, K., Sakai, H., et al., Proc. ODP, Sci. Results, 111: College Station, TX (Ocean Drilling Program), 17-26.

Thompson, R.N., and Humphris, S.E., 1980. Silicate mineralogy of basalts from the East Pacific Rise, OCP Ridge, and Siqueiros fracture zone: Duep Sea Drilling Project Leg 54. In Rosendahl, B.R., Hekinian, R., et al., Init. Repts. DSDP, 54: Washington (U.S. Govt. Printing Office), 651-669.

Tsuchiyama, A., 1983. Crystallization kinetics in the system $\mathrm{CaMgSi}_{2} \mathrm{O}_{6}$ $\mathrm{CaAl}_{2} \mathrm{Si}_{2} \mathrm{O}_{8}$ : the delay in nucleation of diopside and anorthite. Am. Mineral., 68:687-698.

Walker, D., Shibata, T., and Delong, S.E., 1979. Abyssal tholeiites from the Oceanographer Fracture Zone, II: Phase equilibria and mixing. Contrib. Mineral. Petrol., 70:111-125.

Abbreviations for names of organizations and publications in ODP reference lists follow
the style given in Chemical Abstracts Service Source Index (published by American
Chemical Society).

Date of initial receipt: 15 July 1993

Date of acceptance: 18 April 1994

Ms 137/140SR-001 


\begin{tabular}{|c|c|c|c|c|c|c|c|c|c|c|c|c|c|c|c|c|c|c|c|c|c|c|}
\hline \multirow[b]{2}{*}{ Sample } & \multirow[b]{2}{*}{ Grain } & \multicolumn{10}{|c|}{ Weight percent } & \multicolumn{11}{|c|}{ Cations per 6 oxygens } \\
\hline & & $\mathrm{SiO}_{2}$ & $\mathrm{Al}_{2} \mathrm{O}_{3}$ & $\mathrm{FeO}$ & $\mathrm{MnO}$ & $\mathrm{MgO}$ & $\mathrm{CaO}$ & $\mathrm{Na}_{2} \mathrm{O}$ & $\mathrm{TiO}_{2}$ & $\mathrm{Cr}_{2} \mathrm{O}_{3}$ & Total & $\mathrm{Si}$ & $\mathrm{Al}$ & $\mathrm{Fe}$ & $\mathrm{Mn}$ & $\mathrm{Mg}$ & $\mathrm{Ca}$ & $\mathrm{Na}$ & $\mathrm{Ti}$ & $\mathrm{Cr}$ & Total & $\mathrm{Mg} \#$ \\
\hline \multicolumn{23}{|l|}{ 137-504B- } \\
\hline $181 \mathrm{M}-1,115-124 \mathrm{~cm}$ & PH-1 & 52.77 & 3.39 & 6.21 & 0.200 & 18.23 & 18.73 & 0.206 & 0.494 & 0.416 & 100.65 & 1.912 & 0.145 & 0.188 & 0.006 & 0.985 & 0.727 & 0.015 & 0.014 & 0.012 & 4.003 & 84.0 \\
\hline $181 \mathrm{M}-1115-124 \mathrm{~cm}$ & $\mathrm{PH}-1$ & 5313 & 314 & 6.36 & 0171 & 18.43 & 1889 & 0226 & 0.457 & 0.373 & & 1917 & 0134 & 0192 & 0.005 & 0.991 & 0730 & 0.016 & 0012 & 0011 & & 83.8 \\
\hline 1811 & $\mathrm{PH}^{\mathrm{PH}-1}$ & $\begin{array}{l}53.13 \\
53.41\end{array}$ & $\begin{array}{l}3.14 \\
2.80\end{array}$ & $\begin{array}{l}0.30 \\
6.51\end{array}$ & 0.160 & $\begin{array}{l}18.43 \\
19.90\end{array}$ & $\begin{array}{l}18.89 \\
16.98\end{array}$ & 0.224 & 0.427 & 0.413 & & 24 & $\begin{array}{l}0.134 \\
0.119\end{array}$ & $\begin{array}{l}0.192 \\
0.196\end{array}$ & 0.005 & $\begin{array}{l}0.991 \\
1.068\end{array}$ & 55 & $\begin{array}{l}0.016 \\
0.016\end{array}$ & $\begin{array}{l}0.012 \\
0.012\end{array}$ & 0.012 & $\begin{array}{l}4.007 \\
4.007\end{array}$ & $\begin{array}{l}83.8 \\
84.5\end{array}$ \\
\hline $\mathrm{m}$ & $\mathrm{PH}-1$ & 53.74 & 1.59 & 7.17 & 0.205 & 22.15 & 13.73 & 50 & 0.208 & 0.158 & & 7 & & 18 & & & & & 0.006 & .005 & 4.007 & 4.6 \\
\hline $\mathrm{cm}$ & $\mathrm{PH}-1$ & 51.32 & 3.05 & 6.15 & 0.191 & 18.48 & 19.31 & 0.235 & 0.400 & 0.357 & 99 & 1.8 & 0.1 & 0.190 & 0. & i. & 33 & 0.0 & so & 10 & & 4.3 \\
\hline $4 \mathrm{~cm}$ & $\mathrm{PH}-1$ & 52.57 & 2.66 & 6.99 & 0.150 & 18.93 & 18.01 & 0.223 & 0.344 & 0.131 & 00 & $1.2+3$ & 0.115 & 0.214 & 0.005 & 1.c & 05 & 16 & U & & & 8 \\
\hline$-1,115-124 \mathrm{~cm}$ & PH-1r & 52.91 & 2.58 & 8.52 & 0.216 & 18.68 & 17.22 & 0.179 & 0.430 & 0.027 & 100.76 & 1.926 & 0.111 & 0.259 & 0.007 & $\begin{array}{l}1.001 \\
1.013\end{array}$ & 672 & 13 & 0.012 & .001 & & 6 \\
\hline $1,115-124 \mathrm{~cm}$ & $\mathrm{PH}-2$ & 53.43 & 2.69 & 4.99 & 0.145 & 18.47 & 19.77 & 0.265 & 0.221 & 0.609 & 100. & 1.932 & 0.115 & 0.151 & 0.004 & 0.995 & 0.766 & 0.019 & 0.006 & 0.017 & 4.005 & 86.8 \\
\hline 1, $115-124 \mathrm{~cm}$ & $\mathrm{PH}-2$ & 53.30 & 2.80 & 5.04 & 0.229 & 18.55 & 19.56 & 0.253 & 0.261 & 0.605 & 100. & 1.927 & 0.119 & 0.152 & 0.007 & 1.000 & 0.758 & 0.018 & 0.007 & 0.017 & 4.006 & 86.8 \\
\hline $181 \mathrm{M}-1,115-124 \mathrm{~cm}$ & $\mathrm{PH}-2$ & 53.10 & 2.91 & 4.97 & 0.116 & 18.69 & 19.16 & 0.242 & 0.285 & 0.663 & 100.14 & 1.926 & 0.124 & 0.151 & 0.004 & 1.010 & 0.745 & 0.017 & 0.008 & 0.019 & 4.003 & 87.0 \\
\hline $181 \mathrm{M}-1,115-124 \mathrm{~cm}$ & $\mathrm{PH}-2$ & 52.93 & 2.66 & 5.18 & 0.153 & 18.69 & 19.16 & 0.253 & 0.270 & 0.443 & 99.74 & 1.930 & 0.114 & 0.158 & 0.005 & 1.016 & 0.748 & 0.018 & 0.007 & 0.013 & 4.009 & 86.6 \\
\hline $181 \mathrm{M}-1,115-124 \mathrm{~cm}$ & $\mathrm{PH}-2 \mathrm{r}$ & 50.03 & 1.46 & 20.63 & 0.424 & 9.04 & 17.24 & 0.271 & 0.909 & 0.000 & 100.01 & 1.951 & 0.067 & 0.673 & 0.014 & 0.526 & 0.721 & 0.021 & 0.027 & 0.000 & 3.999 & 43.9 \\
\hline $181 \mathrm{M}-1,115-124 \mathrm{~cm}$ & $\mathrm{PH}-3 \mathrm{r}$ & 53.24 & 2.42 & 6.87 & 0.196 & 18.45 & 18.50 & 0.229 & 0.368 & 0.047 & 100.32 & 1.938 & 0.104 & 0.209 & 0.006 & 1.001 & 0.722 & 0.016 & 0.010 & 0.001 & 4.007 & 82.7 \\
\hline $1,115-124 \mathrm{~cm}$ & $\mathrm{PH}-3$ & 53.06 & 3.27 & $\begin{array}{l}0.07 \\
5.29\end{array}$ & 0.164 & 18.35 & 19.36 & 0.256 & 0.291 & 0.752 & 100.80 & $\begin{array}{l}1.930 \\
1.916\end{array}$ & 0.139 & 0.160 & 0.005 & 0.988 & 0.749 & 0.018 & 0.008 & 0.022 & 04 & 86.1 \\
\hline & PH-3 & 52.93 & 2.83 & 5.07 & $\begin{array}{l}0.104 \\
0.129\end{array}$ & 18.33 & $\begin{array}{l}19.50 \\
19.23\end{array}$ & $\begin{array}{l}0.250 \\
0.216\end{array}$ & $\begin{array}{l}0.291 \\
0.260\end{array}$ & 0.573 & 99 & $\begin{array}{l}1.910 \\
1.932\end{array}$ & 0.122 & 0.155 & 0.004 & $\begin{array}{l}0.988 \\
0.997\end{array}$ & 52 & & 0.007 & 0.017 & & $\begin{array}{l}80.1 \\
86.6\end{array}$ \\
\hline & $\mathrm{PH}-3$ & 53.27 & $\begin{array}{l}2.83 \\
2.65\end{array}$ & 5.32 & 0.112 & 18.96 & 18.85 & 0.222 & $\begin{array}{l}0.200 \\
0.239\end{array}$ & & 100 & 1. & $\begin{array}{l}0.122 \\
0.113\end{array}$ & 0 & & $\begin{array}{l}0.991 \\
1.024\end{array}$ & & & 0.007 & 0.019 & & $\begin{array}{l}80.0 \\
86.4\end{array}$ \\
\hline $181 \mathrm{M}-1,115-124 \mathrm{~cm}$ & $\mathrm{PH}-3$ & 53.41 & 2.13 & 5.79 & 0.168 & 19.22 & 18.54 & 0.191 & 0.257 & 0.247 & 99.95 & 1.943 & 0.091 & 0.176 & 0.005 & 1.042 & 0.723 & 0.014 & 0.007 & 0.007 & 4.008 & 85.5 \\
\hline \multirow{2}{*}{\multicolumn{23}{|c|}{$140-504 \mathrm{~B}-$}} \\
\hline & & & & & 0.130 & 1 & 20 & 0. & 0.524 & 0. & 99.64 & 1.886 & 0.165 & 3 & 0. & 0.925 & 07 & 0.017 & 0.015 & 08 & 21 & 8 \\
\hline$-1,18-24 \mathrm{~cm}$ & $\mathrm{PH}-1$ & 49.91 & 4.78 & 6.60 & 0.183 & 16.30 & 19.99 & 0.294 & 0.808 & 0.471 & 99.3 & 1.852 & 0.209 & 0.205 & 0.006 & 0.901 & 0.795 & 0.021 & 0.023 & 0.014 & 4.025 & 81.5 \\
\hline$-1,18-24 \mathrm{~cm}$ & PH-1 & 49.71 & 4.63 & 6.57 & 0.157 & 15.94 & 20.12 & 0.250 & 0.787 & 0.487 & 98.66 & 1.858 & 0.204 & 0.205 & 0.005 & 0.888 & 0.806 & 0.018 & 0.022 & 0.014 & 4.020 & 81.2 \\
\hline $\mathrm{-1}, 18-24 \mathrm{~cm}$ & $\mathrm{PH}-1$ & 50.89 & 4.28 & 6.74 & 0.148 & 16.94 & 19.32 & 0.230 & 0.547 & 0.511 & 99.6 & 1.877 & 0.186 & 0.208 & 0.005 & 0.931 & 0.764 & 0.016 & 0.015 & 0.015 & 4.016 & 81.8 \\
\hline $4 \mathrm{~cm}$ & $\mathrm{PH}-1$ & 49.90 & 4.65 & 6.70 & 0.130 & 16.29 & 19.64 & 0.183 & 0.630 & 0.581 & 98.7 . & 1.861 & 0.2 & 0.209 & 0.004 & 0.905 & 0.785 & 0.013 & 0.018 & 0.017 & & 81.2 \\
\hline $\mathrm{cm}$ & $\mathrm{PH}-2$ & 50.12 & 4.29 & 6.98 & 0.2 & 16.33 & 19.50 & 0.238 & 0.672 & 0.574 & 98. & & 0.1 & 0.218 & & 0.9 & & & 0.019 & 0.017 & & 80.6 \\
\hline & $\mathrm{PH}-2$ & 50.62 & 3.97 & 7.38 & 0. & 16.57 & 19.29 & 0.215 & 0.622 & 0.5 & 70 & & 0.1 & 0.229 & & 0. & & & 0.017 & & & 0 \\
\hline & PH-2 & 50 & 3. & $\begin{array}{l}7.38 \\
7.40\end{array}$ & 0. & & 19 & & 0. & & & & 0.1 & 0 & & 0. & & & 0.017 & & & .0 \\
\hline & & & 3. & 7 & & & & & & & & & 0.1 & & & 0. & & & 0. & $\begin{array}{l}0.011 \\
0.010\end{array}$ & $\begin{array}{l}4.031 \\
4.028\end{array}$ & \\
\hline 2 & $\begin{array}{l}\mathrm{PH}-2 \\
\mathrm{PH}-2\end{array}$ & $\begin{array}{l}50.50 \\
50.78\end{array}$ & & $\begin{array}{l}7.84 \\
7.91\end{array}$ & 0.2 & & & $\begin{array}{l}0.211 \\
0.257\end{array}$ & $\begin{array}{l}0.023 \\
0.672\end{array}$ & & & & 0.1 & $\begin{array}{l}0.243 \\
0.247\end{array}$ & & 0. & 0. & & 0. & $\begin{array}{l}0.010 \\
0.004\end{array}$ & $\begin{array}{l}4.028 \\
4.024\end{array}$ & 1.4 \\
\hline 2 & $\mathrm{PH}-2$ & 51.09 & 2.96 & 8.37 & 0.324 & 16.90 & 18.76 & 0.259 & 0.611 & & 99 & & 0.1 & 0.260 & & 0. & 0. & & & 0.003 & 4 & 78.3 \\
\hline & $\mathrm{PH}$ & 50.70 & 2,67 & 8.74 & 0.250 & & $\begin{array}{l}17.73 \\
17.73\end{array}$ & 0.224 & $\begin{array}{l}0.526 \\
0.526\end{array}$ & & 98 & & 0.1 & 0.275 & & 0 & 5 & 0 & 0. & 0.001 & & 77.9 \\
\hline & & $3 x$ & & 9 & & & $\begin{array}{l}17 \\
18\end{array}$ & & 0. & & & & 0. & & & & & & & & & \\
\hline & $\mathrm{Pl}$ & & & & & & & & & & & & & & & & & & & & & \\
\hline & $\mathrm{Pl}$ & 51 & 2 & & 0. & 17 & 14 & 0. & & 0. & & & & & & & & & & & & \\
\hline n & & & 1. & & 0 . & & 15 & & 0. & & & & 0 & 0. & & & & & & & & 42.5 \\
\hline 1 & & 51 & 1. & & 0 . & & 14 & & 0 & & 10 & & 0. & & & & & & 0.8 & 0. & & 57.1 \\
\hline 1,8 & & 51. & 3. & & 0. & 15 & 20. & & 0. & & & i. & 0.1 & 0. & & 0.8 & 0.814 & & 0.016 & 0.014 & & 81.6 \\
\hline 8 & $\mathrm{Pl}$ & 52 & 3 & & 0 & 16 & 20.55 & 0 & 0.5 & 0. & 100 & & 0.139 & 0.197 & 0.006 & 0.8 & 0.803 & 0.021 & 0.016 & & 4.000 & 81.8 \\
\hline 10 & $\mathrm{PH}-1$ & 53.66 & 2 & & 0.1 & 16.41 & 19.52 & 0.269 & 0.453 & 0.2 & 100 & & 0.125 & 0.208 & 0.006 & 0.889 & 0.761 & 0.019 & 0.012 & 0.008 & 3.979 & 81.1 \\
\hline ont & $\mathrm{PH}-1$ & 52.76 & 2.8 & 6.71 & 0.187 & 16.66 & 19.61 & 0.243 & 0.426 & 0.2 & & & 0.125 & 0.206 & 0.006 & 0.912 & 0.772 & 0.017 & 0.012 & 0.006 & 3.994 & 81.6 \\
\hline 2 & PH-1 & 52.83 & $\begin{array}{l}2.89 \\
2.76\end{array}$ & $\begin{array}{l}0.71 \\
7.55\end{array}$ & 0.195 & $\begin{array}{l}10.00 \\
17.14\end{array}$ & $\begin{array}{l}19.01 \\
18.66\end{array}$ & $\begin{array}{l}0.243 \\
0.230\end{array}$ & $\begin{array}{l}0.420 \\
0.438\end{array}$ & $\begin{array}{l}0.209 \\
0.146\end{array}$ & 99. & $\begin{array}{l}1.938 \\
1.937\end{array}$ & $\begin{array}{l}0.125 \\
0.120\end{array}$ & $\begin{array}{l}0.200 \\
0.232\end{array}$ & $\begin{array}{l}0.000 \\
0.006\end{array}$ & $\begin{array}{l}0.912 \\
0.937\end{array}$ & 0.733 & 0.016 & 0.012 & $\begin{array}{l}0.000 \\
0.004\end{array}$ & 3.9 & $\begin{array}{l}81.0 \\
80.2\end{array}$ \\
\hline $8-12$ & $\begin{array}{l}\mathrm{PH}-1 \\
\mathrm{PH}-1 \mathrm{r}\end{array}$ & $\begin{array}{l}52.83 \\
50.89\end{array}$ & $\begin{array}{l}2.10 \\
1.16\end{array}$ & $\begin{array}{l}7.53 \\
21.68\end{array}$ & $\begin{array}{l}0.195 \\
0.570\end{array}$ & 12.17 & $\begin{array}{l}18.00 \\
12.73\end{array}$ & $\begin{array}{l}0.230 \\
0.242\end{array}$ & $\begin{array}{l}0.438 \\
0.748\end{array}$ & $\begin{array}{l}0.146 \\
0.008\end{array}$ & 100. & $\begin{array}{l}1.931 \\
1.962\end{array}$ & $\begin{array}{l}0.120 \\
0.053\end{array}$ & $\begin{array}{l}0.232 \\
0.699\end{array}$ & $\begin{array}{l}0.000 \\
0.019\end{array}$ & $\begin{array}{l}0.937 \\
0.699\end{array}$ & $\begin{array}{l}0.133 \\
0.526\end{array}$ & $\begin{array}{l}0.010 \\
0.018\end{array}$ & 0.022 & $\begin{array}{l}0.004 \\
0.000\end{array}$ & $\begin{array}{l}3.997 \\
3.999\end{array}$ & $\begin{array}{l}80.2 \\
50.0\end{array}$ \\
\hline-12 & $\mathrm{PH}-2$ & 52.27 & 3.84 & 6.29 & 0.178 & 16.38 & 19.62 & 0.320 & 0.523 & 0.519 & 99 & 1.914 & 0.166 & 0.193 & 0.006 & 0.894 & 0.770 & 0.023 & 0.014 & 0.015 & 3.993 & 82.3 \\
\hline 22 & $\mathrm{PH}-2$ & 51.87 & 4. & 6. & 0.191 & 16.40 & 19.66 & 0.260 & 0.599 & 0.5 & 99 & 1. & 0.173 & 0.196 & 0. & 0.896 & 0.772 & 0. & 0.017 & 0.016 & 3. & 82.0 \\
\hline & & & 3. & & $0.1+2>3$ & & 19.98 & & 0.375 & & (9: & & 0.1 & & & & 0.780 & & 0.010 & 0.012 & & 3.0 \\
\hline & & & & & & & & & & & & & & & & & & & 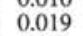 & 1 & 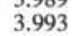 & 9.4 \\
\hline
\end{tabular}


Table 8. Pyroxene microphenocrysts.

\begin{tabular}{|c|c|c|c|c|c|c|c|c|c|c|c|c|c|c|c|c|c|c|c|c|c|c|}
\hline \multirow[b]{2}{*}{ Sample } & \multirow[b]{2}{*}{ Grain } & \multicolumn{10}{|c|}{ Weight percent } & \multicolumn{11}{|c|}{ Cations per 6 oxygens } \\
\hline & & $\mathrm{SiO}_{2}$ & $\mathrm{Al}_{2} \mathrm{O}_{3}$ & $\mathrm{FeO}$ & $\mathrm{MnO}$ & $\mathrm{MgO}$ & $\mathrm{CaO}$ & $\mathrm{Na}_{2} \mathrm{O}$ & $\mathrm{TiO}_{2}$ & $\mathrm{Cr}_{2} \mathrm{O}_{3}$ & Total & $\mathrm{Si}$ & $\mathrm{Al}$ & $\mathrm{Fe}$ & $\mathrm{Mn}$ & $\mathrm{Mg}$ & $\mathrm{Ca}$ & $\mathrm{Na}$ & $\mathrm{Ti}$ & $\mathrm{Cr}$ & Total & $\mathrm{Mg} \#$ \\
\hline \\
\hline $115-1$ & $P-1 r$ & 50.52 & 69 & 19.83 & 0.400 & .83 & 16.74 & 0.299 & 856 & & 101.1 & 1.935 & 0.076 & 0.635 & 13 & 0.618 & 0.687 & 0.022 & 0.025 & 0.000 & 4.013 & 19.3 \\
\hline $\mathrm{cm}$ & MP-1r & 53.25 & 29 & 11.55 & 0.293 & 31 & 13.71 & 0.182 & 0.327 & 15 & 1( & 1. & 0.056 & 4 & 9 & 1.083 & 539 & .013 & 09 & 000 & 4.016 & 75.4 \\
\hline $\mathrm{cm}$ & MP-1 & 54.46 & 1.25 & 8.63 & 0.242 & 21.84 & 13.55 & 0.144 & 0.235 & 43 & 1( & 1.967 & 0.053 & 0.261 & 007 & 5 & 0.524 & 010 & 06 & .001 & 4.005 & 81.9 \\
\hline $115-124 \mathrm{~cm}$ & MP-1 & 52.55 & 3.33 & 6.08 & 0.132 & 17.85 & 19.43 & 0.253 & 0.374 & 0.262 & 100.26 & 1.914 & 0.143 & 0.185 & .004 & 0.969 & 0.758 & 018 & .010 & 0.008 & 4.009 & 84.0 \\
\hline $181 \mathrm{M}-1,115-124 \mathrm{~cm}$ & MP-1 & 52.92 & 2.59 & 6.51 & 0.215 & 19.40 & 17.59 & 0.165 & 0.263 & 0.248 & 99.90 & 1.928 & 0.111 & 0.198 & 0007 & 1.054 & 0.687 & .012 & 0.007 & 0.007 & 4.011 & 84.2 \\
\hline $181 \mathrm{M}-1,115-124 \mathrm{~cm}$ & MP-1 & 54.07 & 1.38 & 7.19 & 0.171 & 21.50 & 15.02 & 0.154 & 0.236 & 0.146 & 99.87 & 1.960 & 0.059 & 0.218 & 0.005 & 1.161 & 0.583 & 0.011 & 0.006 & 0.004 & 4.008 & 84.2 \\
\hline \multicolumn{23}{|l|}{$140-504 \mathrm{~B}-$} \\
\hline 170 . & MP-1 & 51.87 & 2.82 & 8.50 & 0.254 & 17.31 & 18.46 & 0.295 & 0.556 & 0.110 & 100.17 & 1.911 & 0.123 & 0.262 & 0.008 & 0.950 & 0.729 & 0.021 & 0.015 & 0.003 & 4.021 & 78.4 \\
\hline & MP-1 & 1.89 & 05 & 7.41 & 0.217 & & 19.49 & 0.251 & 0.437 & & & & & 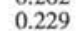 &  & & & & & & & 80.2 \\
\hline 1,1 & MP-1r & 51.91 & 2.21 & $\begin{array}{r}11.41 \\
11.21\end{array}$ & 0.316 & $\begin{array}{l}10.04 \\
16.79\end{array}$ & 16.80 & 0.195 & 0.619 & 0 & 10 & 1.5 & 0. & 0.3 & 0.0 & & & & 0.017 & 0.000 & & 72.7 \\
\hline 1,1 & MP-2 & $\begin{array}{l}31.91 \\
52.02\end{array}$ & $\begin{array}{l}2.21 \\
3.07\end{array}$ & 8.17 & 0.295 & 16.97 & $\begin{array}{l}10.80 \\
18.47\end{array}$ & $\begin{array}{l}0.195 \\
0.283\end{array}$ & $\begin{array}{l}0.019 \\
0.553\end{array}$ & 0.198 & & 1.8 (1. & & 0.2 & 0.0 & & & & 0. & $\begin{array}{l}0.000 \\
0.006\end{array}$ & & 78.7 \\
\hline & MP-2 & 52.35 & 2.74 & 8.34 & 0.267 & 17.59 & 17.86 & 0.294 & 0.506 & 0.183 & 100 & 1.5 & 0.119 & 0.2 & 0.0 & & & t & & 0.005 & 4.012 & \\
\hline & MP-2 & 51.12 & 4.75 & 5.76 & 0.133 & 16.07 & 20.60 & 0.299 & 0.611 & 0.3 & 99. & 1.8 & 0.2 & 0.1 & 0.0 & 0.8 & 0.8 & 0.0 & 0.017 & 0.010 & 4.0 & 83.3 \\
\hline $\mathrm{cm}$ & MP-2 & 52.51 & 2.54 & 9.24 & 0.163 & 17.59 & 17.44 & 0.215 & 0.574 & 0.083 & 100 & 1.928 & 0.1 & 0.2 & 0.0 & 0.9 & 0.6 & 0.0 & 0.016 & 0.002 & 4.0 & 77.2 \\
\hline $1,18-24 \mathrm{~cm}$ & MP-2 & 52.46 & 2.61 & 9.80 & 0.375 & 17.64 & 16.69 & 0.241 & 0.596 & 0.081 & 100.51 & 1.9 & 0.113 & 36 & 0.0 & 0.9 & 0.6 & 17 & 17 & 0.002 & 4.0 & 76.2 \\
\hline l, $18-24 \mathrm{~cm}$ & MP-2 & 52.26 & 3.0 & 8.28 & 0.280 & 17.46 & 18.17 & 0.214 & 0.544 & 0.241 & 100 & 1.9 & 0.1 & 0.254 & 0.0 & 0.9 & 0.713 & 0. & 0.015 & 0.007 & 4.010 & 79.0 \\
\hline $8-12 \mathrm{~cm}$ & MP & 53.60 & 1.3 & 7.87 & 0.215 & 19.13 & 16.55 & 0.223 & 0.257 & 0.115 & 99 & 1.9 & 0.060 & 0.242 & 0.0 & 1.0 & 0.651 & & 0.007 & 0.003 & 4.001 & 81.2 \\
\hline, $8-12 \mathrm{cl}$ & MP-1 & 54.40 & 0.8 & 6.45 & 0.166 & 18.16 & 16.57 & 0.161 & 0.153 & 0.1 & 97. & 2.0 & 0.037 & 0.2 & 0.0 & 1.0 & 0.661 & & 0.004 & 0.003 & 3.956 & 83.4 \\
\hline $8-12 \mathrm{~cm}$ & MP-1 & 54.04 & 1. & 7.46 & 0.252 & 19.28 & 16.69 & 0.231 & 0.253 & 0.119 & 99 & 1.5 & 0.0 & 0.2 & 0.0 & & 0.652 & & 0.007 & 0.003 & 3.997 & 82.2 \\
\hline I. $8-12 \mathrm{cn}$ & MP-1 & 54.11 & 1.5 & 7.65 & 0.235 & 19.74 & 16.03 & 0.158 & 0.229 & 0.278 & 100 & 1.9 & 0.068 & 0.233 & 0.0 & 1.0 & 0.625 & 0. & 0.006 & 0.008 & 3.994 & 82.2 \\
\hline $8-12 \mathrm{cr}$ & MP-2 & 52.85 & 2.66 & 8.39 & 0.352 & 17.03 & 18.10 & 0.286 & 0.497 & 0.065 & 100. & 1.9 & 0.115 & 0.257 & 0.0 & 0.9 & 0.711 & 0 & 0.014 & 0.002 & 4.000 & 78.4 \\
\hline $8-12 c$ & MP-2 & 52.42 & 2.55 & 9.12 & 0.206 & $\begin{array}{l}17.00 \\
0\end{array}$ & $\begin{array}{l}10.10 \\
17.69\end{array}$ & 0.263 & 0.486 & 0.016 & 99 . & 1.5 & 0.1 & 0.2 & 0.0 & 0. & 0 & 9 & 0.6 & 0. & $\begin{array}{l}4.000 \\
4.004\end{array}$ & $\begin{array}{l}10.4 \\
76.9\end{array}$ \\
\hline $8-12$ c & MP-2r & 50.23 & 0.77 & $\begin{array}{r}33.12 \\
23.90\end{array}$ & $\begin{array}{l}0.589 \\
0.589\end{array}$ & $\begin{array}{l}8.62 \\
8.62\end{array}$ & 14.90 & 0.238 & $\begin{array}{l}0.400 \\
0.454\end{array}$ & 0.0 & 99 & 1.5 & 0. & 0.7 & 0.0 & 0. & 0. & 8 & 0.8 & 0. & $\frac{14}{5}$ & 39.1 \\
\hline $8-12$ & MP-3 & $\begin{array}{l}30.23 \\
52.45\end{array}$ & 3.22 & $\begin{array}{r}2.390 \\
7.31\end{array}$ & 0.233 & $\begin{array}{c}.0 .02 \\
16.44\end{array}$ & $\begin{array}{l}14.90 \\
19.25\end{array}$ & 0.326 & $\begin{array}{l}0.434 \\
0.456\end{array}$ & $\begin{array}{l}0.0 \\
0.2\end{array}$ & $\begin{array}{l}99 \\
99\end{array}$ & 1.5 & 0 . & 0.2 & 0.0 & 0. & 0 . & 3 & 0. & 0. & 99 & 80.0 \\
\hline $8-12$ & MP-3 & 52.45 & 2.64 & 7.42 & 0.192 & 15.52 & 20.67 & 0.290 & 0.557 & 0.050 & 99 & 1. & 0 . & 0.2 & 0. & & 0 . & & 0. & 0. & 9 & 78.9 \\
\hline $8-12 c$ & MP-3r & 52.52 & 2.73 & 9.42 & 0.227 & 15.61 & 18.98 & 0.303 & 0.590 & 0.000 & 100 & 1. & 0 . & 0.2 & 0. & & 0. & 2 & 0. & & 3.999 & 74.7 \\
\hline & MP-3r & 51.82 & 2.04 & 13.11 & 0.317 & 14.28 & 17.41 & 0.322 & 0.742 & 0.000 & & & & 0.4 & 0. & & & 3 & 0.021 & & 4.001 & 66.0 \\
\hline $226 \mathrm{R}-1,8-12 \mathrm{~cm}$ & MP-3r & $\begin{array}{l}51.82 \\
50.56\end{array}$ & $\begin{array}{l}2.04 \\
1.10\end{array}$ & 22.91 & 0.544 & 8.85 & 15.76 & 0.286 & 0.735 & 0.000 & 100.76 & 1.968 & 0.051 & 0.746 & 0.018 & 0.514 & 0.657 & 0.022 & 0.022 & 0.000 & 3.996 & 40.8 \\
\hline
\end{tabular}


Table 9. Ophitic pyroxene.

Weight percent

Cations per 6 oxygens

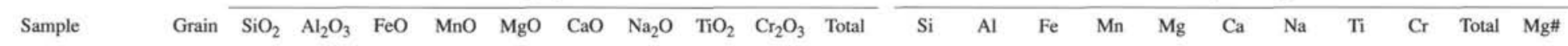

137-504B-

$181 \mathrm{M}-1,115-124 \mathrm{~cm}$

$181 \mathrm{M}-1,115-124 \mathrm{~cm}$

$181 \mathrm{M}-1,115-124 \mathrm{~cm}$
$181 \mathrm{M}-1,115-124 \mathrm{~cm}$

$181 \mathrm{M}-1,115-124 \mathrm{~cm}$

$181 \mathrm{M}-1,115-124 \mathrm{~cm}$

$181 \mathrm{M}-1,115-124 \mathrm{~cm}$
$181 \mathrm{M}-1,115-124 \mathrm{~cm}$

140-504B-

$200 \mathrm{R}-1,18-24 \mathrm{~cm}$

$200 \mathrm{R}-1,18-24 \mathrm{~cm}$

$200 \mathrm{R}-1,18-24 \mathrm{~cm}$

200R-1, $18-24 \mathrm{~cm}$

$200 \mathrm{R}-1,18-24 \mathrm{~cm}$

$200 \mathrm{R}-1,18-24 \mathrm{~cm}$

$200 \mathrm{R}-1,18-24 \mathrm{~cm}$

200R-1, 18-24 cm

$200 \mathrm{R}-1,18-24 \mathrm{~cm}$

$200 \mathrm{R}-1,18-24 \mathrm{~cm}$
$200 \mathrm{R}-1,18-24 \mathrm{~cm}$

$200 \mathrm{R}-1,18-24 \mathrm{~cm}$

$200 \mathrm{R}-1,18-24 \mathrm{~cm}$

200R-1, $18-24 \mathrm{~cm}$

$200 \mathrm{R}-1,18-24 \mathrm{~cm}$
$200 \mathrm{R}-1,18-24 \mathrm{~cm}$

$226 \mathrm{R}-1,8-12 \mathrm{~cm}$

$226 \mathrm{R}-1,8-12 \mathrm{~cm}$

$226 \mathrm{R}-1,8-12 \mathrm{~cm}$

$226 \mathrm{R}-1,8-12 \mathrm{~cm}$

$226 \mathrm{R}-1,8-12 \mathrm{~cm}$

$226 \mathrm{R}-1,8-12 \mathrm{~cm}$

$226 \mathrm{R}-1,8-12 \mathrm{~cm}$

$226 \mathrm{R}-1,8-12 \mathrm{~cm}$

226R-1, 8-12 cm

$226 \mathrm{R}-1,8-12 \mathrm{~cm}$

$\begin{array}{lllllllllll}\text { OP-1r } & 52.00 & 2.39 & 10.90 & 0.284 & 16.94 & 16.78 & 0.219 & 0.498 & 0.017 & 100.03\end{array}$ $\begin{array}{lllllllllll}\text { OP-Ir } & 53.99 & 1.74 & 11.66 & 0.321 & 19.79 & 13.44 & 0.151 & 0.380 & 0.000 & 101.48\end{array}$ $\begin{array}{lllllllllll}\mathrm{OP}-1 & 54.55 & 1.42 & 6.77 & 0.205 & 20.58 & 16.33 & 0.191 & 0.224 & 0.191 & 100.48\end{array}$ $\begin{array}{lllllllllll}\text { OP- } & 54.10 & 1.40 & 6.77 & 0.214 & 20.92 & 15.80 & 0.152 & 0.202 & 0.134 & 99.69\end{array}$ $\begin{array}{lllllllllll}\mathrm{OP}-1 & 52.14 & 2.78 & 7.47 & 0.269 & 17.99 & 17.75 & 0.227 & 0.486 & 0.095 & 99.21\end{array}$ $\begin{array}{lrrrrrrrrrr}\text { OP-1r } & 50.07 & 1.67 & 19.49 & 0.458 & 10.11 & 17.25 & 0.278 & 0.876 & 0.021 & 100.22 \\ \text { OP-1r } & 48.86 & 1.14 & 26.57 & 0.679 & 5.10 & 16.40 & 0.252 & 0.580 & 0.000 & 99.57\end{array}$

$\begin{array}{lllllllllll}\text { OP-1r } & 52.12 & 2.46 & 9.59 & 0.251 & 17.07 & 18.29 & 0.229 & 0.503 & 0.086 & 100.59\end{array}$ $\begin{array}{lllllllllll}\text { OP-1 } & 51.54 & 3.30 & 7.98 & 0.290 & 17.03 & 18.53 & 0.289 & 0.600 & 0.196 & 99.76\end{array}$ $\begin{array}{lllllllllll}\text { OP-1 } & 51.90 & 2.91 & 7.93 & 0.265 & 17.23 & 18.82 & 0.245 & 0.515 & 0.150 & 99.96 \\ \text { OP-1 } & 51.99 & 2.81 & 7.83 & 0.268 & 16.71 & 19.41 & 0.222 & 0.542 & 0.170 & 99.95\end{array}$ $\begin{array}{llllllllllr}\text { OP-1 } & 51.82 & 3.81 & 7.83 & 0.268 & 16.71 & 19.41 & 0.222 & 0.542 & 0.170 & 99.95 \\ \text { OP- } & 51.85 & 0.318 & 17.20 & 18.79 & 0.233 & 0.470 & 0.108 & 100.28\end{array}$ \begin{tabular}{lllllllllllll} 
OP-1 & 51.88 & 2.98 & 7.84 & 0.237 & 16.80 & 18.78 & 0.298 & 0.505 & 0.110 & 99.42 \\
\hline
\end{tabular}

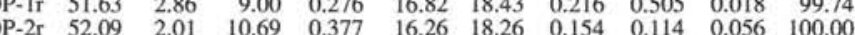
$\begin{array}{llllllllllll}\mathrm{OP}-2 \mathrm{r} & 52.75 & 2.29 & 11.48 & 0.377 & 16.63 & 17.03 & 0.226 & 0.127 & 0.000 & 100.90\end{array}$ \begin{tabular}{llllllllllll}
\hline OP-2 & 52.38 & 2.86 & 8.77 & 0.298 & 16.71 & 18.66 & 0.215 & 0.131 & 0.093 & 100.12
\end{tabular} \begin{tabular}{llllllllllll}
\hline PP-2 & 52.20 & 2.83 & 8.65 & 0.221 & 16.44 & 19.92 & 0.219 & 0.101 & 0.099 & 100.68
\end{tabular} \begin{tabular}{lllllllllll} 
OP-2r & 51.68 & 2.65 & 10.09 & 0.363 & 16.43 & 18.78 & 0.236 & 0.106 & 0.056 & 100.40 \\
\hline OP- $3 \mathrm{r}$ & 49.64 & 0.95 & 25.64 & 0.793 & 8.82 & 14.02 & 0.147 & 0.572 & 0.026 & 100.61
\end{tabular} $\begin{array}{llllllllllll}\mathrm{OP}-3 \mathrm{r} & 50.25 & 1.13 & 22.60 & 0.675 & 1.82 & 14.02 & 0.147 & 0.572 & 0.026 & 100.61\end{array}$ $\begin{array}{lllllllllll}0.3 & 50.58 & 1.40 & 20.52 & 0.508 & 12.13 & 15.12 & 0.259 & 0.786 & 0.031 & 101.33\end{array}$ $\begin{array}{lllllllllll}\mathrm{OP}-3 & 50.04 & 1.62 & 19.59 & 0.509 & 12.33 & 15.38 & 0.305 & 0.900 & 0.020 & 100.68\end{array}$ $\begin{array}{lllllllllll}\text { OP-3 } & 49.91 & 1.80 & 17.79 & 0.494 & 13.10 & 15.39 & 0.242 & 0.909 & 0.096 & 99.72\end{array}$ $\begin{array}{llllllllllll}\mathrm{OP}-3 & 50.35 & 1.94 & 15.71 & 0.330 & 14.19 & 15.56 & 0.264 & 0.775 & 0.021 & 99.15 \\ \mathrm{OP}-3 & 50.92 & 2.02 & 14.74 & 0.350 & 15.05 & 15.57 & 0.288 & 0.794 & 0.059 & 99.79\end{array}$ $\begin{array}{lllllllllll}\text { OP-3 } & 50.92 & 2.02 & 14.74 & 0.350 & 15.05 & 15.57 & 0.288 & 0.794 & 0.059 & 99.79 \\ \text { OP-1 } & 52.90 & 2.34 & 12.46 & 0.334 & 17.21 & 14.79 & 0.233 & 0.628 & 0.000 & 100.89\end{array}$ $\begin{array}{llllllllllll}\mathrm{OP}-1 & 52.94 & 2.50 & 10.89 & 0.3316 & 14.21 & 14.82 & 0.2363 & 0.628 & 0.000 & 10.89\end{array}$ $\begin{array}{lllllllllll}\mathrm{OP}-1 & 51.82 & 2.33 & 12.72 & 0.275 & 13.85 & 18.54 & 0.367 & 0.800 & 0.029 & 100.73\end{array}$ $\begin{array}{lllllllllll}\text { OP-1 } & 52.26 & 1.70 & 16.25 & 0.357 & 13.80 & 15.03 & 0.332 & 0.740 & 0.000 & 100.47\end{array}$ $\begin{array}{lllllllllll}\text { OP-1r } & 51.04 & 1.00 & 21.74 & 0.574 & 9.61 & 16.16 & 0.288 & 0.553 & 0.000 & 100.97\end{array}$ $\begin{array}{llllllllllll}\text { OP-2 } & 53.14 & 3.11 & 7.18 & 0.134 & 16.70 & 18.63 & 0.303 & 0.446 & 0.046 & 99.69\end{array}$ $\begin{array}{lllllllllll}O P P-2 & 54.85 & 1.61 & 8.67 & 0.260 & 18.03 & 16.81 & 0.235 & 0.295 & 0.000 & 100.76\end{array}$ $\begin{array}{lllllllllll}\text { OP-2 } & 53.44 & 2.36 & 8.95 & 0.190 & 16.62 & 17.98 & 0.259 & 0.502 & 0.004 & 100.30 \\ \text { OP-2 } & 53.07 & 2.09 & 12.05 & 0.308 & 15.45 & 16.67 & 0.314 & 0.552 & 0.000 & 100.50\end{array}$ $\begin{array}{lllllllllll}\text { OP-3 } & 51.90 & 1.49 & 18.28 & 0.525 & 11.77 & 15.95 & 0.331 & 0.788 & 0.000 & 101.04\end{array}$ $\begin{array}{lllllllllll}\text { OP-3r } & 50.87 & 0.91 & 24.74 & 0.594 & 8.07 & 13.82 & 0.322 & 0.574 & 0.000 & 99.90\end{array}$ $\begin{array}{lllllllllll}\text { OP-4 } & 53.97 & 1.48 & 7.61 & 0.280 & 17.70 & 16.55 & 0.219 & 0.308 & 0.000 & 98.12 \\ \text { OP-5 } & 52.98 & 2.40 & 8.05 & 0.218 & 16.06 & 18.72 & 0.280 & 0.504 & 0.009 & 99.22\end{array}$

$\begin{array}{lllllllllll}.928 & 0.105 & 0.338 & 0.009 & 0.936 & 0.667 & 0.016 & 0.014 & 0.001 & 4.013 & 73.5\end{array}$ $\begin{array}{llllllllllll}1.967 & 0.07 & 0.353 & 0.010 & 1.068 & 0.521 & 0.011 & 0.010 & 0.000 & 4.003 & 75.2\end{array}$

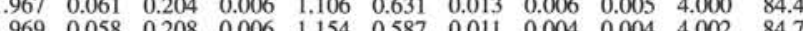
$\begin{array}{llllllllllll} & 964 & 0.060 & 0.206 & 0.007 & 1.132 & 0.615 & 0.011 & 0.006 & 0.004 & 4.004 & 84.6\end{array}$ $\begin{array}{llllllllllll}9.924 & 0.121 & 0.230 & 0.008 & 0.990 & 0.702 & 0.016 & 0.014 & 0.003 & 4.008 & 81.1\end{array}$ $\begin{array}{lllllllllll}1.970 & 0.054 & 0.896 & 0.023 & 0.306 & 0.708 & 0.020 & 0.018 & 0.000 & 3.995 & 25.5\end{array}$

$\begin{array}{lllllllllll}.919 & 0.107 & 0.295 & 0.008 & 0.937 & 0.722 & 0.016 & 0.014 & 0.003 & 4.020 & 76.0\end{array}$ $\begin{array}{llllllllllll}.903 & 0.144 & 0.247 & 0.009 & 0.937 & 0.733 & 0.021 & 0.017 & 0.006 & 4.016 & 79.2 \\ \end{array}$ $\begin{array}{lllllllllllll}918 & 0.122 & 0.242 & 0.008 & 0.941 & 0.743 & 0.018 & 0.014 & 0.004 & 4.017 & 79.5 \\ 1 & 0.136 & 0.016 & 0.015 & 0.005 & 4.012 & 79.2\end{array}$ $\begin{array}{lllllllllllll}.907 & 0.130 & 0.257 & 0.010 & 0.944 & 0.741 & 0.017 & 0.013 & 0.003 & 4.022 & 78.6\end{array}$ $\begin{array}{llllllllllll}1.914 & 0.130 & 0.243 & 0.007 & 0.927 & 0.745 & 0.021 & 0.014 & 0.003 & 4.010 & 79.2 \\ 1.93 & 0.125 & 0.279 & 0.009 & 0.929 & 0.732 & 0.016 & 0.014 & 0.001 & 4.017 & 76.9\end{array}$ $\begin{array}{lllllllllll}1.939 & 0.088 & 0.333 & 0.012 & 0.902 & 0.728 & 0.011 & 0.003 & 0.002 & 4.018 & 73.1\end{array}$ $\begin{array}{lllllllllll}.943 & 0.099 & 0.353 & 0.012 & 0.913 & 0.672 & 0.016 & 0.004 & 0.000 & 4.012 & 72.1\end{array}$ $\begin{array}{lllllllllllll}1.930 & 0.124 & 0.270 & 0.009 & 0.918 & 0.737 & 0.015 & 0.004 & 0.003 & 4.011 & 77.2\end{array}$ \begin{tabular}{lllllllllll}
.916 & 0.116 & 0.313 & 0.011 & 0.907 & 0.785 & 0.016 & 0.003 & 0.003 & 4.023 & 77.2 \\
\hline & 0.017 & 0.003 & 0.002 & 4.031 & 74.4
\end{tabular} $\begin{array}{lllllllllll}9.946 & 0.052 & 0.845 & 0.027 & 0.518 & 0.592 & 0.011 & 0.017 & 0.001 & 4.010 & 38.0\end{array}$ $\begin{array}{lllllllllllll}1.934 & 0.063 & 0.656 & 0.016 & 0.691 & 0.619 & 0.019 & 0.023 & 0.001 & 4.022 & 51.3\end{array}$ $\begin{array}{lllllllllll}1.921 & 0.074 & 0.629 & 0.017 & 0.705 & 0.632 & 0.023 & 0.026 & 0.001 & 4.027 & 52.9\end{array}$ $\begin{array}{lllllllllll}1.919 & 0.082 & 0.572 & 0.016 & 0.751 & 0.634 & 0.018 & 0.026 & 0.003 & 4.021 & 56.7\end{array}$ $\begin{array}{lllllllllll}.926 & 0.088 & 0.503 & 0.011 & 0.809 & 0.638 & 0.020 & 0.022 & 0.001 & 4.017 & 61.7\end{array}$

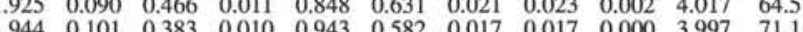
$\begin{array}{lllllllllll}939 & 0.109 & 0.335 & 0.010 & 0.779 & 0.782 & 0.026 & 0.020 & 0.000 & 4.000 & 69.9\end{array}$ $\begin{array}{lllllllllll}935 & 0.103 & 0.397 & 0.009 & 0.770 & 0.741 & 0.027 & 0.022 & 0.001 & 4.005 & 66.0\end{array}$ $\begin{array}{lllllllllll}.966 & 0.075 & 0.511 & 0.011 & 0.774 & 0.606 & 0.024 & 0.021 & 0.000 & 3.988 & 60.2\end{array}$ $\begin{array}{lllllllllll}.972 & 0.046 & 0.703 & 0.019 & 0.554 & 0.669 & 0.022 & 0.016 & 0.000 & 4.000 & 44.1\end{array}$ $\begin{array}{llllllllllll}1.94 & 0.134 & 0.220 & 0.004 & 0.912 & 0.731 & 0.022 & 0.012 & 0.001 & 3.984 & 80.6\end{array}$ $\begin{array}{lllllllllllll}.958 & 0.102 & 0.274 & 0.006 & 0.908 & 0.706 & 0.018 & 0.014 & 0.000 & 3.986 & 76.8\end{array}$ $\begin{array}{lllllllllll}0.091 & 0.373 & 0.010 & 0.852 & 0.661 & 0.023 & 0.015 & 0.000 & 3.987 & 69.6\end{array}$ $\begin{array}{llllllllll}0.067 & 0.579 & 0.017 & 0.665 & 0.648 & 0.024 & 0.023 & 0.000 & 3.989 & 53.4\end{array}$ $\begin{array}{lllllllll}0.579 & 0.012 & 0.665 & 0.648 & 0.024 & 0.023 & 0.000 & 3.989 & 3.4\end{array}$ $\begin{array}{lllllllllll}1.960 & 0.105 & 0.249 & 0.007 & 0.886 & 0.742 & 0.020 & 0.014 & 0.000 & 3.983 & 78.1\end{array}$ 
Table 10. Poikilitic pyroxene.

Weight percent

Cations per 6 oxyge

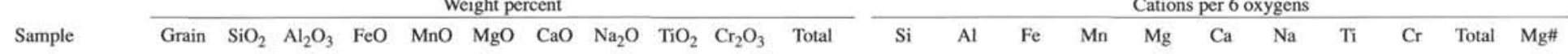

137-504B-

$\begin{array}{llllllllllll}181 \mathrm{M}-1,115-124 \mathrm{~cm} & \text { PO-1r } & 48.55 & 1.31 & 28.44 & 0.691 & 4.08 & 16.66 & 0.236 & 0.956 & 0.000 & 100.92\end{array}$ $\begin{array}{lllllllllllll}181 \mathrm{M}-1,115-124 \mathrm{~cm} & \text { PO-1r } & 49.16 & 1.59 & 25.32 & 0.600 & 6.48 & 16.75 & 0.246 & 1 . & 0.004 & 101.29 \\ 181 \mathrm{M}-1,115-124 \mathrm{~cm} & \text { PO-1 } & 50.09 & 1.80 & 21.57 & 0.447 & 11.52 & 14.23 & 0.308 & 0.878 & 0.000 & 100.84\end{array}$ $\begin{array}{llllllllllll}181 \mathrm{M}-1,115-124 \mathrm{~cm} & \text { PO-1 } & 49.97 & 1.76 & 22.48 & 0.463 & 10.94 & 14.16 & 0.263 & 0.845 & 0.000 & 100.88\end{array}$

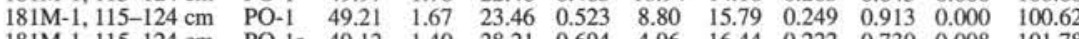
$\begin{array}{llllllllllll}181 \mathrm{M}-1,115-124 \mathrm{~cm} & \text { PO-1r } & 49.12 & 1.40 & 28.21 & 0.694 & 4.96 & 16.44 & 0.222 & 0.730 & 0.008 & 101.78 \\ 181 \mathrm{M}-1,115-124 \mathrm{~cm} & \text { PO-2r } & 48.91 & 1.39 & 25.71 & 0.665 & 6.99 & 16.02 & 0.231 & 0.991 & 0.000 & 100.91\end{array}$ $\begin{array}{lllllllllllll}181 \mathrm{M}-1.115-124 \mathrm{~cm} & \mathrm{PO}-2 \mathrm{r} & 58.04 & 1.39 & 25.73 & 0.665 & 5.99 & 16.02 & 0.231 & 0.991 & 0.000 & 100.91\end{array}$

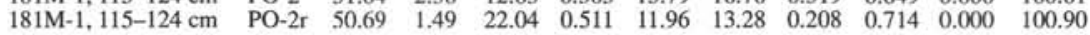
140-504B-

$200 \mathrm{R}-1,18-24 \mathrm{~cm}$

$200 \mathrm{R}-1,18-24 \mathrm{~cm}$

$200 \mathrm{R}-1,18-24 \mathrm{~cm}$

$200 \mathrm{R}-1,18-24 \mathrm{~cm}$

$200 \mathrm{R}-1,18-24 \mathrm{~cm}$

$200 \mathrm{R}-1,18-24 \mathrm{~cm}$
$200 \mathrm{R}-1,18-24 \mathrm{~cm}$

$200 \mathrm{R}-1,18-24 \mathrm{~cm}$

$200 \mathrm{R}-1,18-24 \mathrm{~cm}$

$200 \mathrm{R}-1,18-24 \mathrm{~cm}$

$200 \mathrm{R}-1,18-24 \mathrm{~cm}$

$200 \mathrm{R}-1,18-24 \mathrm{~cm}$

$200 \mathrm{R}-1,18-24 \mathrm{~cm}$

$200 \mathrm{R}-1,18-24 \mathrm{~cm}$

200R-1, 18-24 cm

200R-1, $18-24 \mathrm{~cm}$

$200 \mathrm{R}-1,18-24 \mathrm{~cm}$

$200 \mathrm{R}-1,18-24 \mathrm{~cm}$

$200 \mathrm{R}-1,18-24 \mathrm{~cm}$

$200 \mathrm{R}-1,18-24 \mathrm{~cm}$

200R-1, $18-24 \mathrm{~cm}$

200R-1, $18-24 \mathrm{~cm}$

200R-1, 18-24 cm

$226 \mathrm{R}-1,8-12 \mathrm{~cm}$

$226 \mathrm{R}-1,8-12 \mathrm{~cm}$

$226 \mathrm{R}-1,8-12 \mathrm{~cm}$

$226 \mathrm{R}-1,8-12 \mathrm{~cm}$

226R-1, 8-12 cm

$226 \mathrm{R}-1,8-12 \mathrm{~cm}$

$226 \mathrm{R}-1,8-12 \mathrm{~cm}$

226R $-1,8-12 \mathrm{~cm}$

226R-1, 8-12 cm

$226 \mathrm{R}-1,8-12 \mathrm{~cm}$

226R-1, 8-12 cm

$226 \mathrm{R}-1,8-12 \mathrm{~cm}$

$226 \mathrm{R}-1,8-12 \mathrm{~cm}$

$226 \mathrm{R}-1,8-12 \mathrm{~cm}$

$226 \mathrm{R}-1,8-12 \mathrm{~cm}$

$226 \mathrm{R}-1,8-12 \mathrm{~cm}$

$226 \mathrm{R}-1,8-12 \mathrm{~cm}$
$226 \mathrm{R}-1,8-12 \mathrm{~cm}$

$226 \mathrm{R}-1,8-12 \mathrm{~cm}$
$226 \mathrm{R}-1,8-12 \mathrm{~cm}$

$\begin{array}{lllllllllll}\text { PO-1 } & 50.71 & 4.96 & 6.05 & 0.194 & 16.67 & 19.78 & 0.287 & 0.927 & 0.517 & 100.10\end{array}$ $\begin{array}{lllllllllll}\mathrm{PO}-1 & 51.98 & 4.05 & 6.36 & 0.115 & 16.49 & 20.06 & 0.218 & 0.590 & 0.409 & 100.28\end{array}$ $\begin{array}{lrlllllllllr}\text { PO-1 } & 51.21 & 4.30 & 5.66 & 0.150 & 16.37 & 20.64 & 0.227 & 0.661 & 0.347 & 99.5 \\ \text { PO-1 } & 52.25 & 4.05 & 6.13 & 0.215 & 17.06 & 20.16 & 0.244 & 0.520 & 0.176 & 100.80\end{array}$ $\begin{array}{lllllllllllll}\text { PO-1 } & 51.23 & 4.28 & 6.92 & 0.170 & 16.66 & 19.55 & 0.276 & 0.887 & 0.335 & 100.31\end{array}$ $\begin{array}{lllllllllll}\text { PO-1 } & 51.26 & 4.23 & 6.50 & 0.172 & 16.68 & 19.65 & 0.209 & 0.846 & 0.392 & 99.94\end{array}$ $\begin{array}{lllllllllll}\text { PO-1r } & 50.22 & 2.23 & 12.47 & 0.371 & 16.14 & 15.99 & 0.228 & 0.612 & 0.013 & 98.27 \\ \text { PO-1r } & 52.97 & 2.41 & 15.56 & 0.218 & 14.98 & 11.93 & 0.401 & 0.599 & 0.019 & 99.00\end{array}$ \begin{tabular}{llllllllllll}
\hline PO-Ir & 42.37 & 1.47 & 2.56 & 0.218 & 14.98 & 1.93 & 0.401 & 0.599 & 0.019 & 99.09
\end{tabular} $\begin{array}{llllllllllll}\text { PO-1r } & 51.39 & 2.46 & 20.13 & 0.292 & 11.59 & 11.62 & 0.393 & 0.230 & 0.012 & 98.12\end{array}$ $\begin{array}{lllllllllll}\text { PO-2 } & 51.22 & 4.01 & 6.52 & 0.176 & 16.77 & 20.10 & 0.315 & 0.648 & 0.225 & 99.99\end{array}$ $\begin{array}{lllllllllll}\text { PO-2 } & 51.87 & 3.25 & 6.71 & 0.224 & 17.56 & 18.80 & 0.205 & 0.685 & 0.257 & 99.56\end{array}$ $\begin{array}{lllllllllll}\mathrm{PO}-2 & 52.58 & 3.47 & 7.43 & 0.212 & 18.41 & 18.68 & 0.211 & 0.530 & 0.305 & 101.82\end{array}$

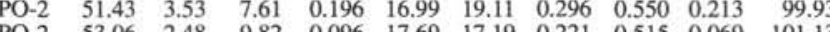

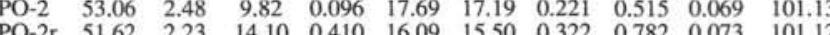
$\begin{array}{lllllllllll}\text { PO-2r } & 49.45 & 1.30 & 20.69 & 0.516 & 10.38 & 16.20 & 0.258 & 0.759 & 0.019 & 99.57\end{array}$ $\begin{array}{lllllllllll}\text { PO-3r } & 49.57 & 1.23 & 22.26 & 0.613 & 9.74 & 15.24 & 0.193 & 0.726 & 0.022 & 99.59\end{array}$ $\begin{array}{lllllllllll}\text { PO-3r } & 50.69 & 1.17 & 24.12 & 0.584 & 11.91 & 12.01 & 0.169 & 0.704 & 0.000 & 101.36\end{array}$ $\begin{array}{lllllllllll}\text { PO-3r } & 50.20 & 1.09 & 23.79 & 0.688 & 11.07 & 12.67 & 0.142 & 0.636 & 0.000 & 100.27\end{array}$ \begin{tabular}{lllllllllll}
\hline $0-3$ & 52.53 & 2.35 & 10.42 & 0.237 & 17.81 & 16.15 & 0.199 & 0.531 & 0.038 & 100.27
\end{tabular} $\begin{array}{lllllllllll}\mathrm{PO}-3 & 52.61 & 2.53 & 8.85 & 0.281 & 17.77 & 17.54 & 0.278 & 0.512 & 0.074 & 100.45\end{array}$ \begin{tabular}{llllllllllll}
\hline & -3 & 52.07 & 3.41 & 7.89 & 0.2198 & 17.34 & 18.57 & 0.228 & 0.567 & 0.413 & 100.69
\end{tabular} $\begin{array}{lllllllllll}\text { PO-3 } & 52.72 & 3.80 & 7.44 & 0.181 & 16.61 & 19.72 & 0.242 & 0.107 & 0.437 & 101.25\end{array}$ $\begin{array}{lllllllllll}\text { PO-3 } & 51.71 & 4.13 & 6.47 & 0.194 & 15.83 & 21.38 & 0.190 & 0.158 & 0.291 & 100.35\end{array}$ $\begin{array}{lllllllllll}\text { PO-1r } & 50.38 & 1.16 & 23.75 & 0.593 & 8.77 & 14.94 & 0.301 & 0.651 & 0.000 & 100.54\end{array}$

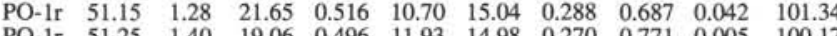

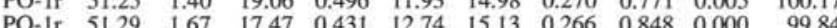
\begin{tabular}{lllllllllll}
\hline PO- 1 & 51.88 & 2.41 & 13.09 & 0.292 & 15.39 & 16.20 & 0.345 & 0.716 & 0.000 & 100.33
\end{tabular} $\begin{array}{llllllllllll}\text { PO-1 } & 52.98 & 2.33 & 10.93 & 0.317 & 16.79 & 16.44 & 0.258 & 0.570 & 0.003 & 100.62\end{array}$ $\begin{array}{llllllllllll}\text { PO-1 } & 52.69 & 2.98 & 6.67 & 0.222 & 17.19 & 18.55 & 0.269 & 0.424 & 0.286 & 99.27\end{array}$ $\begin{array}{lllllllllll}\mathrm{PO}-2 & 52.85 & 2.55 & 7.46 & 0.253 & 17.03 & 18.44 & 0.268 & 0.528 & 0.033 & 99.4\end{array}$ $\begin{array}{llllllllllll}\mathrm{PO}-3 & 50.67 & 1.39 & 23.12 & 0.630 & 1.57 & 14.08 & 0.258 & 0.798 & 0.000 & 101.5\end{array}$ \begin{tabular}{lllllllllllll}
\hline$P 0-3$ & 51.88 & 1.46 & 20.43 & 0.606 & 12.83 & 1.89 & 0.227 & 0.697 & 0.004 & 100.13
\end{tabular} $\begin{array}{llllllllllll}\text { PO-4 } & 52.59 & 3.69 & 6.17 & 0.188 & 15.95 & 20.77 & 0.290 & 0.442 & 0.095 & 100.19\end{array}$ $\begin{array}{lllllllllll}\mathrm{PO}-4 & 52.84 & 3.16 & 6.81 & 0.212 & 16.14 & 19.79 & 0.255 & 0.490 & 0.039 & 99.74\end{array}$ $\begin{array}{lllllllllll}\text { PO-4 } & 53.18 & 2.67 & 9.14 & 0.283 & 16.59 & 17.51 & 0.277 & 0.539 & 0.000 & 100.19\end{array}$ $\begin{array}{lllllllllll}\text { PO-4 } & 52.76 & 2.43 & 11.35 & 0.450 & 16.77 & 15.62 & 0.285 & 0.553 & 0.011 & 100.23\end{array}$ \begin{tabular}{lllllllllllll}
\hline $\mathrm{PO}-4$ & 52.7 & 2.27 & 11.43 & 0.400 & 16.15 & 15.51 & 0.272 & 0.648 & 0.001 & 100.27
\end{tabular} \begin{tabular}{lllllllllll}
\hline $0-4$ & 52.51 & 2.27 & 12.00 & 0.293 & 15.71 & 16.53 & 0.262 & 0.700 & 0.000 & 100.28
\end{tabular} $\begin{array}{llllllllllll}P O-5 r & 52.88 & 2.10 & 9.88 & 0.280 & 15.67 & 16.46 & 0.298 & 0.527 & 0.000 & 98.09\end{array}$ $\begin{array}{lllllllllllll}\text { PO- } 5 & 53.29 & 2.88 & 7.27 & 0.263 & 16.37 & 18.35 & 0.276 & 0.406 & 0.033 & 98.15\end{array}$ \begin{tabular}{lllllllllll} 
PO-5 & 53.04 & 3.31 & 5.79 & 0.143 & 15.71 & 19.81 & 0.272 & 0.438 & 0.066 & 98.58 \\
\hline & 50.91 & 3.65 & 5.24 & 0.129 & 16.44 & 19.27 & 0.257 & 0.458 & 0.127 & 98.47
\end{tabular} $\begin{array}{lllllllllll}\text { PO-5 } & 52.91 & 3.65 & 5.24 & 0.129 & 16.44 & 19.27 & 0.257 & 0.458 & 0.127 & 98.47\end{array}$ $\begin{array}{lllllllllll}\text { PO-5 } & 53.18 & 3.31 & 5.82 & 0.124 & 15.79 & 19.85 & 0.269 & 0.591 & 0.049 & 98.99 \\ \text { PO-5r } & 51.26 & 1.56 & 18.27 & 0.413 & 11.34 & 15.41 & 0.343 & 0.968 & 0.005 & 99.57\end{array}$ $\begin{array}{lllllllllll}1.950 & 0.062 & 0.955 & 0.024 & 0.244 & 0.717 & 0.018 & 0.029 & 0.000 & 3.999 & 20.4\end{array}$ $\begin{array}{lllllllllll}1.929 & 0.082 & 0.695 & 0.015 & 0.661 & 0.787 & 0.019 & 0.034 & 0.000 & 4.003 & 31.3 \\ 1.025 & 0.000 & 4.016 & 48.8\end{array}$ $\begin{array}{lllllllllll}.931 & 0.080 & 0.727 & 0.015 & 0.630 & 0.586 & 0.020 & 0.025 & 0.000 & 4.014 & 46.4\end{array}$

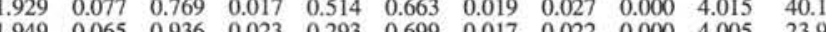
$\begin{array}{llllllllllll}1.949 & 0.065 & 0.936 & 0.023 & 0.293 & 0.699 & 0.017 & 0.022 & 0.000 & 4.005 & 23.9 \\ 1.935 & 0.065 & 0.851 & 0.022 & 0.412 & 0.679 & 0.018 & 0.030 & 0.000 & 4.012 & 32.6\end{array}$ $\begin{array}{lllllllllll}1.914 & 0.105 & 0.402 & 0.010 & 0.883 & 0.671 & 0.023 & 0.018 & 0.000 & 4.027 & 68.7\end{array}$ $\begin{array}{lllllllllll}1.947 & 0.068 & 0.708 & 0.017 & 0.685 & 0.547 & 0.016 & 0.021 & 0.000 & 4.006 & 49.2\end{array}$

$\begin{array}{lllllllllll}1.858 & 0.214 & 0.185 & 0.006 & 0.911 & 0.777 & 0.020 & 0.026 & 0.015 & 4.012 & 83.1\end{array}$

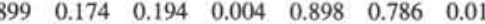
$\begin{array}{llllllllllll}.885 & 0.186 & 0.174 & 0.005 & 0.898 & 0.814 & 0.016 & 0.018 & 0.010 & 4.007 & 83.8 \\ .897 & 0.174 & 0.186 & 0.007 & 0.924 & 0.784 & 0.017 & 0.014 & 0.005 & 4.008 & 83.2\end{array}$ $\begin{array}{lllllllllll}1.877 & 0.185 & 0.212 & 0.005 & 0.910 & 0.768 & 0.020 & 0.024 & 0.010 & 4.011 & 81.1 \\ 1882 & 0.183 & 0.200 & 0.005 & 0.912 & 0.773 & 0.015 & 0.023 & 0.011 & 4.005 & 82.1\end{array}$ $\begin{array}{llllllllllll}.914 & 0.100 & 0.397 & 0.012 & 0.917 & 0.653 & 0.017 & 0.018 & 0.000 & 4.027 & 69.8\end{array}$ $\begin{array}{llllllllllll}1.922 & 0.064 & 0.714 & 0.016 & 0.616 & 0.662 & 0.018 & 0.0121 & 0.000 & 4.034 & 46.3\end{array}$ $\begin{array}{lllllllllll}1.993 & 0.112 & 0.653 & 0.010 & 0.670 & 0.483 & 0.030 & 0.007 & 0.000 & 3.958 & 50.6\end{array}$ $\begin{array}{lllllllllll}1.883 & 0.174 & 0.200 & 0.006 & 0.919 & 0.792 & 0.023 & 0.018 & 0.007 & 4.020 & 82.1\end{array}$ $\begin{array}{lllllllllll}1.908 & 0.141 & 0.206 & 0.007 & 0.963 & 0.741 & 0.015 & 0.019 & 0.008 & 4.007 & 82.3\end{array}$ $\begin{array}{lllllllllll}1.895 & 0.147 & 0.224 & 0.007 & 0.989 & 0.721 & 0.015 & 0.014 & 0.009 & 4.020 & 81.5\end{array}$

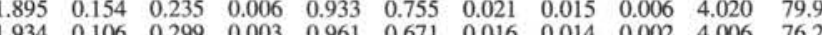
$\begin{array}{lllllllllllll}1.9318 & 0.098 & 0.438 & 0.013 & 0.891 & 0.617 & 0.023 & 0.022 & 0.002 & 4.006 & 76.2\end{array}$ $\begin{array}{lllllllllll}1.937 & 0.060 & 0.678 & 0.017 & 0.606 & 0.680 & 0.020 & 0.022 & 0.001 & 4.020 & 47.2\end{array}$ $\begin{array}{lllllllllll}1.949 & 0.057 & 0.732 & 0.020 & 0.571 & 0.642 & 0.015 & 0.022 & 0.001 & 4.008 & 43.8\end{array}$ $\begin{array}{lllllllllll}1.950 & 0.053 & 0.776 & 0.019 & 0.683 & 0.495 & 0.013 & 0.020 & 0.000 & 4.009 & 46.8\end{array}$ $\begin{array}{lllllllllll}1.956 & 0.050 & 0.775 & 0.023 & 0.643 & 0.529 & 0.011 & 0.019 & 0.000 & 4.006 & 45.3\end{array}$ $\begin{array}{lllllllllll}1.934 & 0.102 & 0.321 & 0.007 & 0.977 & 0.637 & 0.014 & 0.015 & 0.001 & 4.007 & 75.3\end{array}$ $\begin{array}{lllllllllll}1.928 & 0.109 & 0.271 & 0.009 & 0.970 & 0.689 & 0.020 & 0.014 & 0.002 & 4.012 & 78.2\end{array}$ $\begin{array}{llllllllllll}1.902 & 0.147 & 0.241 & 0.006 & 0.944 & 0.727 & 0.016 & 0.016 & 0.012 & 4.011 & 79.7\end{array}$ $\begin{array}{lllllllllll}1.891 & 0.170 & 0.229 & 0.007 & 0.921 & 0.738 & 0.021 & 0.020 & 0.012 & 4.009 & 80.1\end{array}$ $\begin{array}{lllllllllll}1.913 & 0.162 & 0.226 & 0.006 & 0.899 & 0.767 & 0.017 & 0.003 & 0.013 & 4.005 & 79.9\end{array}$ $\begin{array}{lllllllllll}1.897 & 0.179 & 0.199 & 0.006 & 0.865 & 0.840 & 0.014 & 0.004 & 0.008 & 4.012 & 81.3 \\ 1.969 & 0.053 & 0.776 & 0.020 & 0.511 & 0.626 & 0.023 & 0.019 & 0.000 & 3.997 & 39.7\end{array}$ $\begin{array}{lllllllllll}1.969 & 0.053 & 0.776 & 0.020 & 0.511 & 0.626 & 0.023 & 0.019 & 0.000 & 3.997 & 39.7 \\ 1.961 & 0.058 & 0.694 & 0.017 & 0.611 & 0.618 & 0.021 & 0.020 & 0.001 & 4.001 & 46.8\end{array}$ $\begin{array}{lllllllllll}1.961 & 0.058 & 0.694 & 0.017 & 0.611 & 0.618 & 0.021 & 0.020 & 0.001 & 4.001 & 46.8 \\ 1.964 & 0.063 & 0.611 & 0.016 & 0.681 & 0.615 & 0.020 & 0.022 & 0.000 & 3.993 & 52.7\end{array}$ $\begin{array}{llllllllllll}1.957 & 0.075 & 0.557 & 0.014 & 0.725 & 0.619 & 0.020 & 0.024 & 0.000 & 3.991 & 56.5\end{array}$ $\begin{array}{llllllllllll}1.934 & 0.106 & 0.408 & 0.009 & 0.855 & 0.647 & 0.025 & 0.020 & 0.000 & 4.005 & 67.7\end{array}$ $\begin{array}{lllllllllll}1.948 & 0.101 & 0.336 & 0.010 & 0.920 & 0.647 & 0.018 & 0.016 & 0.000 & 3.996 & 73.2\end{array}$ $\begin{array}{lllllllllll}1.938 & 0.129 & 0.205 & 0.007 & 0.942 & 0.731 & 0.019 & 0.012 & 0.008 & 3.991 & 82.1\end{array}$ $\begin{array}{lllllllllll}1.947 & 0.111 & 0.230 & 0.008 & 0.935 & 0.728 & 0.019 & 0.015 & 0.001 & 3.992 & 80.3 \\ 1.950 & 0.063 & 0.744 & 0.021 & 0.606 & 0.581 & 0.019 & 0.023 & 0.000 & 4.006 & 44.9\end{array}$ $\begin{array}{llllllllllllll}1.961 & 0.064 & 0.689 & 0.020 & 0.735 & 0.490 & 0.017 & 0.020 & 0.000 & 3.995 & 51.6\end{array}$ $\begin{array}{llllllllllll}1.923 & 0.159 & 0.189 & 0.006 & 0.869 & 0.814 & 0.021 & 0.012 & 0.003 & 3.995 & 82.2\end{array}$ $\begin{array}{lllllllllll}1.940 & 0.137 & 0.209 & 0.007 & 0.883 & 0.779 & 0.018 & 0.014 & 0.001 & 3.987 & 80.9\end{array}$ $\begin{array}{lllllllllll}1.951 & 0.115 & 0.280 & 0.009 & 0.907 & 0.689 & 0.020 & 0.015 & 0.000 & 3.986 & 76.4\end{array}$ $\begin{array}{lllllllllll}1.948 & 0.106 & 0.350 & 0.014 & 0.923 & 0.618 & 0.020 & 0.015 & 0.000 & 3.994 & 72.5\end{array}$ $\begin{array}{lllllllllll}1.948 & 0.099 & 0.372 & 0.009 & 0.868 & 0.657 & 0.019 & 0.020 & 0.000 & 3.992 & 70.8\end{array}$ $\begin{array}{lllllll}0.746 & 0.561 & 0.015 & 0.020 & 0.000 & 3.984 & 55.6\end{array}$ $\begin{array}{lllllllllll}1.962 & 0.125 & 0.224 & 0.008 & 0.876 & 0.661 & 0.022 & 0.015 & 0.000 & 3.967 & 73.9 \\ 1.9724 & 0.020 & 0.011 & 0.001 & 3.974 & 80.1\end{array}$ $\begin{array}{lllllllllll}.958 & 0.144 & 0.179 & 0.005 & 0.864 & 0.784 & 0.020 & 0.012 & 0.002 & 3.967 & 82.9\end{array}$ $\begin{array}{lllllllllll}1.948 & 0.158 & 0.162 & 0.004 & 0.902 & 0.760 & 0.018 & 0.013 & 0.004 & 3.968 & 84.8\end{array}$ $\begin{array}{lllllllllll}1.955 & 0.143 & 0.179 & 0.004 & 0.865 & 0.782 & 0.019 & 0.016 & 0.001 & 3.966 & 82.9 \\ 1.970 & 0.071 & 0.587 & 0.013 & 0.650 & 0.634 & 0.026 & 0.028 & 0.000 & 3.979 & 52.5\end{array}$ 
Table 11. Olivine phenocrysts.

\begin{tabular}{|c|c|c|c|c|c|c|c|c|c|c|c|c|c|c|c|c|c|c|c|c|c|c|}
\hline \multirow[b]{2}{*}{ Sample } & \multirow[b]{2}{*}{ Grain } & \multicolumn{10}{|c|}{ Weight percent } & \multicolumn{11}{|c|}{ Cations per 4 oxygens } \\
\hline & & $\mathrm{SiO}_{2}$ & $\mathrm{Al}_{2} \mathrm{O}_{3}$ & $\mathrm{FeO}$ & $\mathrm{MnO}$ & $\mathrm{MgO}$ & $\mathrm{CaO}$ & $\mathrm{Na} 2 \mathrm{O}$ & $\mathrm{TiO}_{2}$ & $\mathrm{Cr}_{2} \mathrm{O}_{3}$ & Total & $\mathrm{Si}$ & $\mathrm{Al}$ & $\mathrm{Fe}$ & Mn & $\mathrm{Mg}$ & $\mathrm{Ca}$ & $\mathrm{Na}$ & $\mathrm{Ti}$ & $\mathrm{Cr}$ & Total & Mg\# \\
\hline \multicolumn{23}{|l|}{ 140-504B- } \\
\hline $200 \mathrm{R}-1,18-2$ & $\mathrm{OL}-1$ & 40.46 & 0.059 & 12.49 & 0.214 & 47.71 & 0.039 & 0.000 & 0.000 & 0.038 & 101.02 & 0.994 & 0.0016 & 0.257 & 0.0045 & 1.747 & 0.0011 & 0.0000 & 0.0000 & 0.0007 & 3.005 & 87.2 \\
\hline $18-24 \mathrm{~cm}$ & UL-1 & $\begin{array}{l}40.40 \\
40.26\end{array}$ & 0.059 & 12.51 & $\begin{array}{l}0.214 \\
0.217\end{array}$ & 47.52 & $\begin{array}{l}0.039 \\
0.039\end{array}$ & 0.001 & 0.000 & 0.074 & 100.68 & 0.993 & 0.0017 & 0.258 & 0.0045 & $\begin{array}{l}1.747 \\
1.746\end{array}$ & 0.0 & 0.0 & 0.0 & 0.0014 & 3.006 & 87.1 \\
\hline $18-24 \mathrm{~cm}$ & OL-1 & $\begin{array}{l}40.20 \\
40.21\end{array}$ & 0.039 & 12.47 & 0.209 & 47.17 & $\begin{array}{l}0.059 \\
0.059\end{array}$ & $\begin{array}{l}0.000 \\
0.000\end{array}$ & 0.000 & $\begin{array}{l}0.074 \\
0.048\end{array}$ & $\begin{array}{l}100.08 \\
100.20\end{array}$ & & 0.0012 & 0.258 & 0.0044 & 1.741 & & & & 9 & 3.003 & 1 \\
\hline & $\mathrm{OL}-2$ & 38.93 & 0.020 & 11.31 & 0.185 & 47.45 & 0.029 & 0.001 & 0.011 & & 97.97 & . & & 0.239 & 0.0040 & & & & & & 3.016 & 2 \\
\hline & $\mathrm{OL}-2$ & 38.59 & 0.049 & 11.46 & 0.242 & 47.87 & 0.049 & 0.033 & 0.000 & 0 & 98 & 0.974 & 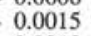 & 0.242 & 2 & 0 & & & & & 26 & 2 \\
\hline m & $\mathrm{OL}-2$ & 40.22 & 0.029 & 12.45 & 0.282 & 47.65 & 0.039 & 0.000 & 0.001 & 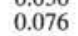 & 10 & 001 & 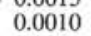 & 0. & 99 & & & & & & 008 & 87.2 \\
\hline $200 \mathrm{R}-1,18-24 \mathrm{~cm}$ & $\mathrm{OL}-2$ & 39.85 & 0.039 & 12.56 & 0.284 & 47.32 & 0.039 & 0.000 & 0.000 & 0.066 & 100.15 & 0.989 & 0.0010 & 0.261 & 0 & 51 & & & & & & 87.0 \\
\hline $200 \mathrm{R}-1,18-24 \mathrm{~cm}$ & $\mathrm{OL}-3$ & 40.07 & 0.029 & 14.15 & 0.289 & 46.20 & 0.039 & 0.000 & 0.000 & 0.028 & 100.80 & 0.994 & 0.0007 & 0.294 & 0.0061 & $\begin{array}{l}1.709 \\
1.709\end{array}$ & 0.0009 & 0.0000 & 0.0000 & 0.0005 & 3.005 & 85.3 \\
\hline $200 \mathrm{R}-1,18-24 \mathrm{~cm}$ & $\mathrm{OL}-3$ & 40.05 & 0.029 & 14.04 & 0.174 & 46.41 & 0.049 & 0.016 & 0.007 & 0.034 & 100.81 & 0.993 & 0.0007 & 0.291 & 0.0037 & 1.715 & 0.0013 & 0.0008 & 0.0 & 0.0007 & 3.007 & 85.5 \\
\hline $200 \mathrm{R}-1,18-24 \mathrm{~cm}$ & $\mathrm{OL}-3$ & 39.43 & 0.020 & 13.80 & 0.220 & 45.91 & 0.059 & 0.005 & 0.014 & 0.019 & 99.47 & 0.5 & 0.0005 & 0.290 & 0.0047 & 1.720 & 0.0 & 0.0 & & 0.0004 & 3.008 & 6 \\
\hline $1,18-24 \mathrm{~cm}$ & OL -4 & 39.55 & 0.029 & 14.59 & 0.299 & 44.93 & 0.049 & 0.009 & 0.000 & 0.000 & 99.46 & 0.5 & 0.0010 & 0.308 & 0.0064 & 1.688 & 0.0 & & & 0. & 3.003 & 6 \\
\hline $18-24 \mathrm{~cm}$ & OL -4 & 39.78 & 0.000 & 15.18 & 0.177 & 45.00 & 0.059 & 0.000 & 0.000 & 0.061 & 100.25 & 0.9 & 0.0000 & 0.318 & 0.0 & 1.681 & & & & 0.0 & 3.002 & \\
\hline $8-12 \mathrm{~cm}$ & 0 & 41.17 & 0.026 & 11.93 & 0.204 & 47.08 & 0.309 & 0.011 & 0. & 0.053 & 100 & 1. & 77 & 0.245 & 0.0042 & & 0.0 & & & 0.0 & & \\
\hline 810 & $\mathrm{O}$ & $\begin{array}{l}41.17 \\
41.12\end{array}$ & 0.043 & 12.11 & 0.208 & $\begin{array}{l}46.72 \\
46.72\end{array}$ & 0.299 & 0.005 & & 0.029 & 100 & i. 0 & & 0.249 & 0.0043 & 1.713 & 0.00 & & & 0.0 & 2 & .0 \\
\hline 22 & $\mathrm{O}$ & $\begin{array}{l}41.12 \\
40.08\end{array}$ & 0.051 & 12.80 & 0.236 & $\begin{array}{l}45.53 \\
45.53\end{array}$ & $\begin{array}{l}0.299 \\
0.315\end{array}$ & 0.005 & & 0.056 & 99.07 & 1.006 & 0.0015 & $\begin{array}{l}0.269 \\
0.269\end{array}$ & 0.0050 & 1.703 & 0.00 & & & & 2 & 4 \\
\hline 22 & o & $\begin{array}{l}40.08 \\
40.26\end{array}$ & $\begin{array}{l}0.051 \\
0.014\end{array}$ & $\begin{array}{l}15.80 \\
15.45\end{array}$ & $\begin{array}{l}0.236 \\
0.248\end{array}$ & $\begin{array}{l}34.33 \\
44.01\end{array}$ & $\begin{array}{l}0.315 \\
0.354\end{array}$ & $\begin{array}{l}0.0022 \\
0.022\end{array}$ & 0.001 & $\begin{array}{l}0.036 \\
0.042\end{array}$ & 100 & $\begin{array}{l}1.000 \\
1.008\end{array}$ & 0.0 & $\begin{array}{l}0.209 \\
0.324\end{array}$ & 0.0 & 1.643 & 0.0 & & & 0.6 & 2.992 & .5 \\
\hline$-12 \mathrm{~cm}$ & $\mathrm{OL}-2$ & 38.33 & 0.025 & 26.80 & 0.405 & 34.79 & 0.030 & 0.000 & 0.016 & 0.003 & 100 & 1.0 & & 0.592 & 0.0 & 1.370 & 0.0 & & & 0. & & 8 \\
\hline & & 40.76 & 0.050 & 12.67 & 0.209 & 45.86 & 0.050 & 0.021 & & & & & & 0.2 & & & & & & & & \\
\hline & & 41 & 0.055 & 12.32 & & & & & & & & & & & & & & & & & & \\
\hline n & & & 0. & 25 & & & & & & & & & & & & & & & & & & \\
\hline n & & 40 & & 14 & & 44 & & & & & & & & & & & & & & & & \\
\hline & Ol & 40.45 & 0. & 13.51 & 0. & 45 & 0.0 & & & 0. & & & & & & & & & & & & 85.7 \\
\hline $226 \mathrm{R}-1,8-12 \mathrm{~cm}$ & $\mathrm{OL}-4$ & $\begin{array}{l}41.19 \\
41.19\end{array}$ & 0.045 & 12.29 & 0.157 & $\begin{array}{l}46.33 \\
46.33\end{array}$ & 0.027 & 0.000 & 0.000 & 0.010 & 100.05 & 1.017 & 0.0013 & 0.254 & 0.0033 & 1.705 & 0.0007 & 0.000 & 0000 & 0.0002 & 2.982 & 87.0 \\
\hline
\end{tabular}

